\title{
EBR-II Data Digitization
}

\author{
Su-Jong Yoon, Cristian Rabiti, \\ John Sackett
}

August 2014

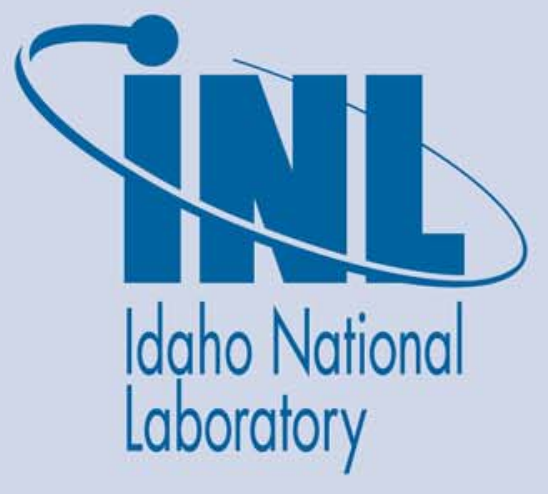

The INL is a U.S. Department of Energy National Laboratory operated by Battelle Energy Alliance 


\section{DISCLAIMER}

This information was prepared as an account of work sponsored by an agency of the U.S. Government. Neither the U.S. Government nor any agency thereof, nor any of their employees, makes any warranty, expressed or implied, or assumes any legal liability or responsibility for the accuracy, completeness, or usefulness, of any information, apparatus, product, or process disclosed, or represents that its use would not infringe privately owned rights. References herein to any specific commercial product, process, or service by trade name, trade mark, manufacturer, or otherwise, does not necessarily constitute or imply its endorsement, recommendation, or favoring by the U.S. Government or any agency thereof. The views and opinions of authors expressed herein do not necessarily state or reflect those of the U.S. Government or any agency thereof. 


\title{
EBR-II Data Digitization
}

\author{
Su-Jong Yoon, \\ Cristian Rabiti, \\ John Sackett
}

August 2014

Idaho National Laboratory Idaho Falls, Idaho 83415

\author{
http://www.inl.gov
}

\author{
Prepared for the \\ U.S. Department of Energy \\ Office of Nuclear Energy \\ Under DOE Idaho Operations Office \\ Contract DE-AC07-05ID14517
}





\begin{abstract}
This work is aimed to initiate a process to generate a validation database from the collection of the operational data of the last nine years (1985-1994) of activity of the EBR-II reactor. In particular two tasks are here accomplished. First all the drawings of the reactor vessel internals, and primary loop are identified in terms of Idaho National Laboratory (INL) Electronic Document Management System (EDMS) Identification Number. Second, a detailed description of all the sensors and their positioning in the reactor is reported to allow for an accurate reproduction of the signals by numerical methodology.
\end{abstract}




\section{CONTENTS}

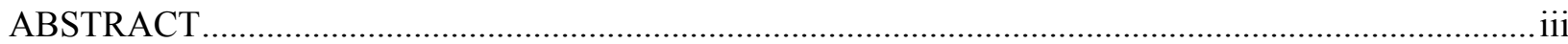

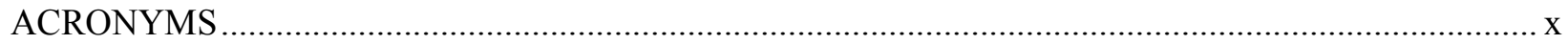

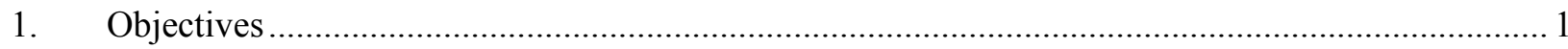

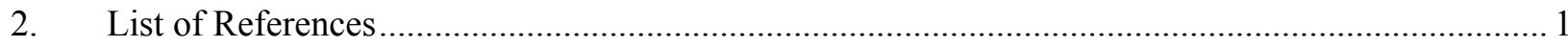

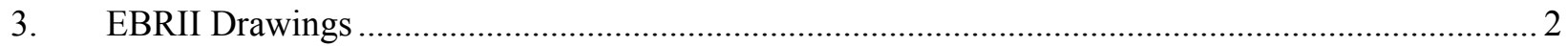

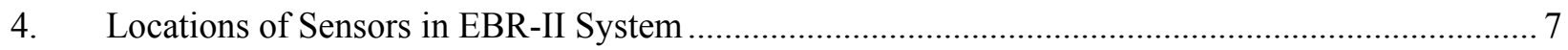

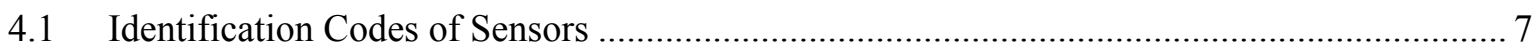

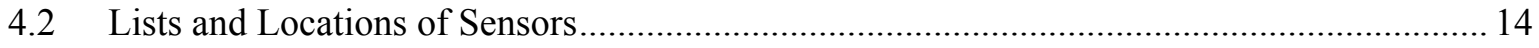

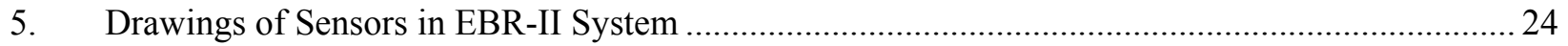

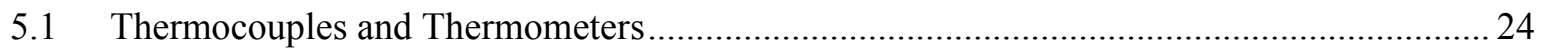

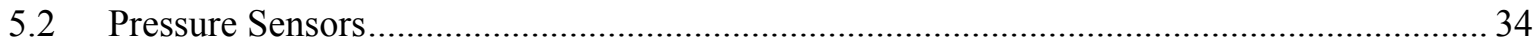

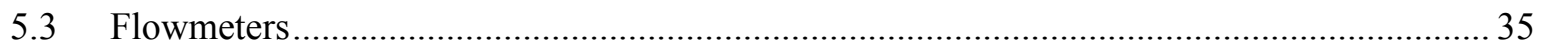

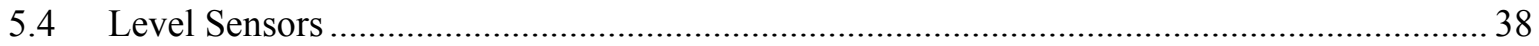

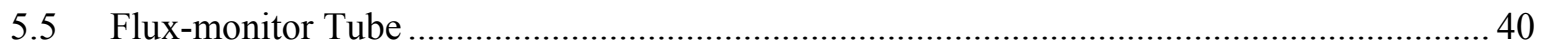

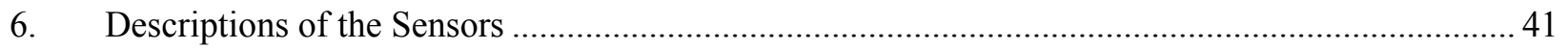

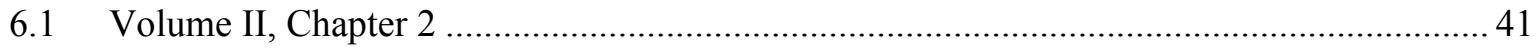

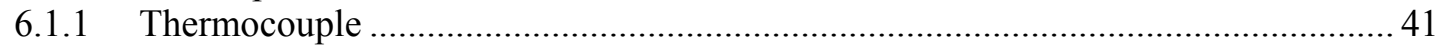

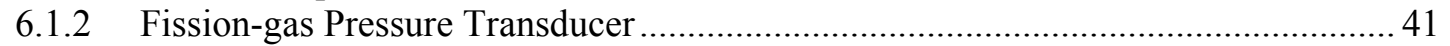

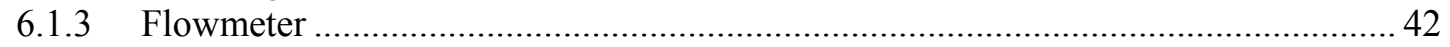

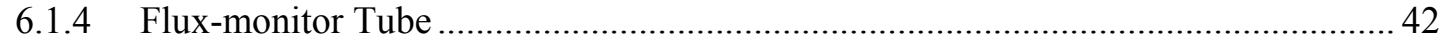

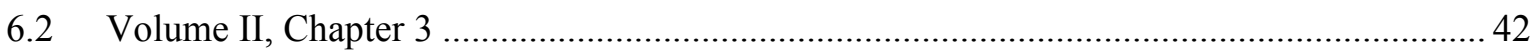

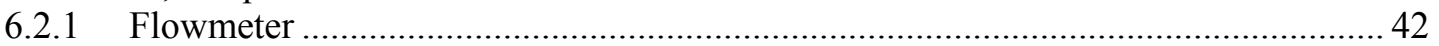

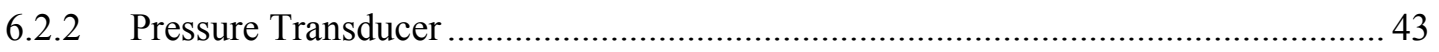

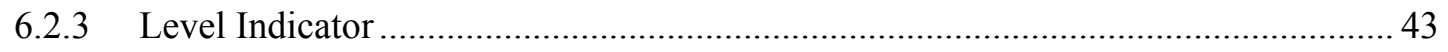

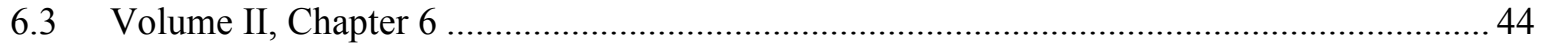

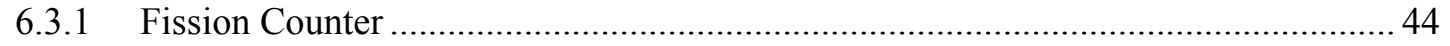

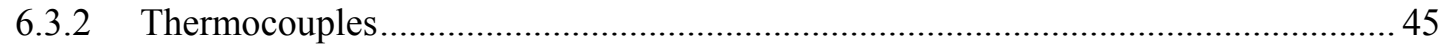

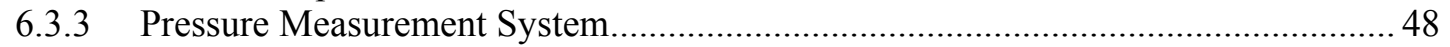

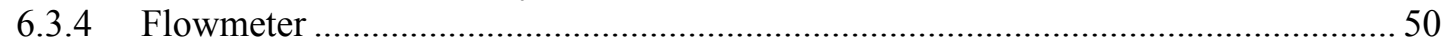

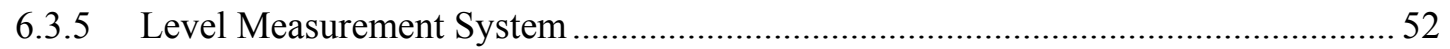

7. Identification of Initial Conditions for the Available Recorded Data …....................................... 54

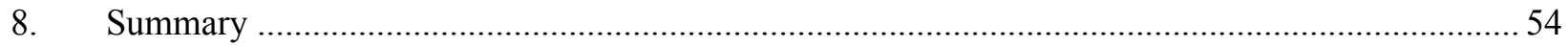

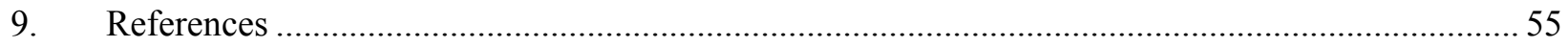




\section{FIGURES}

Figure 1 Figure 6.1-1 EBR-II Flow Diagram [Vol. II, Chapter 6, pp.6.1-7/8] ..................................... 12

Figure 2 Figure 6.3-25 Primary Cooling System Instrumentation [Vol. II, Chapter 6, p.6.3-57] ............ 13

Figure 3 Figure 4.6-5 Primary Tank Nozzles (or Figure 6.3-24 Instrument Lead Penetration through Primary Tank Cover) [Vol. II, Chapter 4, p.4.6-11 or Vol. II, Chapter 6, pp.6.3-55/56] 15

Figure 4 Figure 6.3-1 Nuclear Instrument Thimbles and Thimble Cooling [Vol. II, Chapter 6, pp.6.3-13/14].

Figure 5 Figure 7.4-5 “O” Instrument Thimble Assembly (showing Original Instrumentation Arrangement) [Vol. II, Chapter 7, p.7.4-11] 3

Figure 6 Figure 7.4-6 "J-1" Instrument Thimble Assembly (showing Original Instrumentation Arrangement) [Vol. II, Chapter 7, p.7.4-14] 4

Figure 7 Figure 7.4-7 Original Instrument Cage Area Arrangement J-2, J-3 and J-4 Thimbles [Vol. II, Chapter 7, p.7.4-16]..... 5

Figure 8 Figure 6.3-5 Instrument Locations in Reactor Vessel [Vol. II, Chapter 6, pp.6.3-19/20]........... 6

Figure 9 Figure 6.3-6 Reactor Vessel and Neutron Shield [Vol. II, Chapter 6, pp.6.3-21/22]................. 8

Figure 10 Figure 6.3-7 Primary Pump No.1 [Vol. II, Chapter 6, pp.6.3-23/24] ..................................... 10

Figure 11 Figure 6.3-8 Primary Pump No.2 [Vol. II, Chapter 6, pp.6.3-25/26] .................................... 12

Figure 12 Figure 6.3-9 Bulk Sodium and Argon Sensors in Primary Tank [Vol. II, Chapter 6, pp.6.3-27/28] 14

Figure 13 Figure 6.3-10 Thermocouples on Primary Tank [Vol. II, Chapter 6, pp.6.3-29/30] ............... 16

Figure 14 Figure 6.3-11 Intermediate Heat Exchanger [Vol. II, Chapter 6, pp.6.3-33/34] ...................2 21

Figure 15 Figure 6.3-18 Shutdown Coolers [Vol. II, Chapter 6, pp.6.3-43/44] ...................................23

Figure 16 Figure 6.9-2 Pipe Surface Thermocouple Assembly [Vol. II, Chapter 6, p.6.9-5].................25

Figure 17 Figure 6.9-3 Pipe Surface Thermocouple Mounting [Vol. II, Chapter 6, p.6.9-6]..................26

Figure 18 Figure 6.9-4 Thermocouple Assembly for Thermowell [Vol. II, Chapter 6, p.6.9-7].............27

Figure 19 Figure 6.9-5 Typical Thermowell [Vol. II, Chapter 6, p.6.9-9] ..........................................28

Figure 20 Figure 6.9-6 Typical Thermowell Type Thermocouple Mounting [Vol. II, Chapter 6, p.6.9-10] .29

Figure 21 Figure 6.9-7 Special Thermocouple Assembly [Vol. II, Chapter 6, p.6.9-11] ...................... 30

Figure 22 Figure 6.9-8 Special Thermocouple [Vol. II, Chapter 6, p.6.9-13] 31

Figure 23 Figure 6.9-35 Typical Resistance Thermometer for High Temperature Use in Sodium

[Vol. II, Chapter 6, p.6.9-103] 32

Figure 24 Figure 6.9-36 Resistance Thermometer for Sensing Air Temperatures [Vol. II, Chapter 6, p.6.9-103] 33

Figure 25 Figure 6.10-2 NaK Filled Volumetric Pressure Sensing Element [Vol. II, Chapter 6, p.6.10-5] 34

Figure 26 Figure 6.10-5 Differential Pressure Sensing Head [Vol. II, Chapter 6, p.6.10-13]. 34 
Figure 27 Figure 6.11-1 Magnetic Flowmeter and Flow Tube for Large Sodium Coolant Piping

[Vol. II, Chapter 6, p.6.11-3]

Figure 28 Figure 6.11-8 Small Magnetic Flowmeter Installation - Sodium Purification

Flowmeters, R1-FM-515-A and R1-FM-515-B [Vol. II, Chapter 6, p.6.11-17] .36

Figure 29 Figure 6.15-27 Magnetic Flowmeter [Vol. II, Chapter 6, p.6.15-77]

Figure 30 Figure 6.12-3 Differential Pressure Type Level Transmitter [Vol. II, Chapter 6, p.6.12-7] 38

Figure 31 Figure 6.12-4 Float Type Level Transmitter [Vol. II, Chapter 6, p.6.12-9] ... .39

Figure 32 Figure 2.11-11 Installation of Flux-monitor Tube [Vol. II, Chapter 2, p.2.11-28] 


\section{TABLES}

Table 1 Library IDs of Instruments and Components in Vessels and Internals......................................... 2

Table 2 Library IDs for Instrument in Primary Loop .............................................................................. 6

Table 3 Identification Code Structure [Vol. II, Chapter 6, P.6.3-5] .......................................................... 7

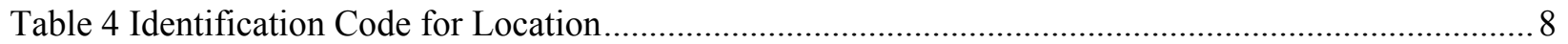

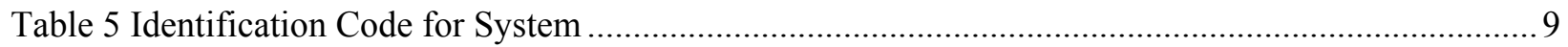

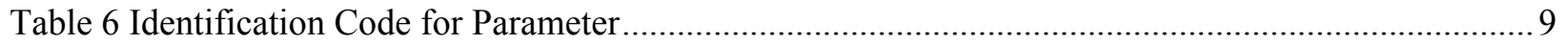

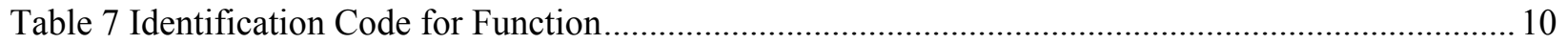

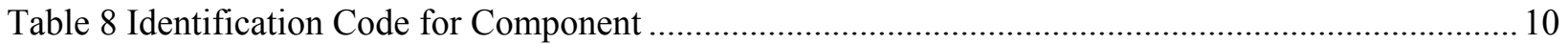

Table 9 Figure 4.6-5 Primary Tank Nozzles (Figure 6.3-24 Instrument Lead Penetration through Primary Tank Cover) [Vol. II, Chapter 4, p.4.6-11 or Vol. II, Chapter 6, pp.6.3$55 / 56]$

Table 10 Figure 6.3-1 Nuclear Instrument Thimbles and Thimble Cooling [Vol. II, Chapter6,

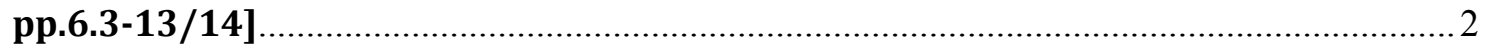

Table 11 Figure 6.3-5 Instrument Locations in Reactor Vessel [Vol. II, Chapter 6, pp. 6.3-19/20] ......... 7

Table 12 Figure 6.3-5 Instrument Locations in Reactor Vessel [Vol. II, Chapter 6, pp. 6.319/20] 7

Table 13 Figure 6.3-6 Reactor Vessel and Neutron Shield [Vol. II, Chapter 6, pp.6.3-21/22]......... 9

Table 14 Figure 6.3-7 Primary Pump No.1 [Vol. II, Chapter 6, pp.6.3-23/24] .............................. 11

Table 15 Figure 6.3-8 Primary Pump No.2 [Vol. II, Chapter 6, pp.6.3-25/26] ................................ 13

Table 16 Figure 6.3-9 Bulk Sodium and Argon Sensors in Primary Tank [Vol. II, Chapter 6,

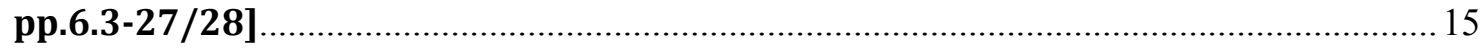

Table 17 Figure 6.3-10 Thermocouples on Primary Tank [Vol. II, Chapter 6, pp.6.3-29/30] $(1 / 4)$

Figure 6.3-10 Thermocouples on Primary Tank [Vol. II, Chapter 6, pp.6.3-

29/30] (2/4)

Figure 6.3-10 Thermocouples on Primary Tank [Vol. II, Chapter 6, pp.6.3-

29/30] (3/4)

Figure 6.3-10 Thermocouples on Primary Tank [Vol. II, Chapter 6, pp.6.329/30] (4/4) 20

Table 18 Figure 6.3-11 Intermediate Heat Exchanger [Vol. II, Chapter 6, pp.6.3-33/34] .............. 22

Table 19 Figure 6.3-18 Shutdown Coolers [Vol. II, Chapter 6, pp.6.3-43/44] 24 


\section{ACRONYMS}

\begin{tabular}{|c|c|}
\hline ANL & Argonne National Laboratory \\
\hline AMS & Average Magnitude Squared \\
\hline BFTF & Breached Fuel Test Facility \\
\hline cps & Counter Per Second \\
\hline DAS & Digital data Acquisition System \\
\hline DND & Delayed Neutron Detector \\
\hline EBR & Experimental Breeder Reactor \\
\hline $\mathrm{FCF}$ & Fuel Cycle Facility \\
\hline FEF & Fuels and Examination Facility \\
\hline FERD & Fuel Element Rupture Detector \\
\hline FFTS & Failed Fuel Transfer System \\
\hline FGM & Fission Gas Monitor \\
\hline FPTF & Fuel performance Test Facility \\
\hline FUM & Fuel Unloading Machine \\
\hline GLASS & Germanium-Lithium Argon-Scanning System \\
\hline IBC & Interbuilding coffin \\
\hline IHX & Intermediate Heat Exchanger \\
\hline INCOT & In-core Instrument Test Facility \\
\hline INSAT & Instrumented Subassembly Test Facility \\
\hline LCR & Log Count Rate \\
\hline LMFBR & Liquid Metals Fast Breeder Reactor \\
\hline MSA & Mine Safety Appliances \\
\hline NIFT & Nuclear Instrument Test Facility \\
\hline NRTS & National Reactor Test Station \\
\hline RCGM & Reactor Cover Gas Monitor \\
\hline RSCL & Radioactive Sodium Chemistry Loop \\
\hline TIR & Test Instrument Room \\
\hline TREAT & Transient Reactor Test Facility \\
\hline
\end{tabular}




\section{EBR-II Data Digitization}

\section{Objectives}

The goal of this report is to place the fundaments of the construction of a validation database based on operating experience of EBR II reactor.

More in detail this report has two objective, first to provide the information to assess the amount of work it will be required to generate CAD files from the EBR-II drawings, and second to provide the information needed to locate and characterize the sensors used to monitor the reaction operation.

The location and availability of the EBR-II drawings is investigated and where possible provided, more detailed information is provided for the sensors.

For the sensors, the document reports a rather detailed information collection, so that this document could be used directly to characterize the sensors both in term of response and location.

\section{List of References}

The following reports have been recorder, as part of this work, in electronic format and used as source of the information for the report.

1. EBR-II System Design Descriptions - Volume I, General Facilities [1]

2. EBR-II System Design Descriptions - Volume II, Primary System [2]
a. Chapter 1. General Information
b. Chapter 2. Reactor
c. Chapter 3. Primary Cooling System
d. Chapter 4. Primary Tank Assembly
e. Chapter 5. Fuel Handling System
f. Chapter 6. Instrumentation
g. Chapter 7. Auxiliary Systems

3. EBR-II System Design Descriptions - Volume III, Secondary System [3]

4. EBR-II System Design Descriptions - Volume IV, Steam System [4]

5. EBR-II System Design Descriptions - Volume V, Electrical System [5]

6. EBR-II System Design Descriptions - Volume VI, Sensitive Systems [6]

In particular "EBR-II System Design Descriptions - Volume II" (Chapters 2 to 5) have been used to identify the IDs of the drawings that will need to be made into CAD files.

The information of the instrumentation in EBR-II primary system is stored in the "EBR-II System Design Descriptions - Volume II, Chapter 6. Instrumentation.”

Along the text square brackets are used to identify the volume that is the source of the reported information. 


\section{EBRII Drawings}

Drawing tables are cataloged and possibly electronically stored in the Idaho National Laboratory Electronic Document Management System (EDMS). EDMS uses the original drawing IDs (referred here as Alternate ID) and its own ID system. The "EBR-II System Design Descriptions - Volume II" has been used as source to collect the "Alternate ID" of the drawing of interest and the search engine of EDMS has been used to find, when possible the new IDs.

Table 1 reports the title, EDMS ID, and Alternate ID of the drawing of interest for the instrument and component in the vessel and internals, while Table 2 reports the same information for the primary loop.

In both tables, the red color indicate drawings that where not found referenced in the EDMS at all, the bold is used to indicate active drawings, and the standard characters indicate inactive drawings. Inactive drawings are drawings that have been cataloged but not made available in electronic format.

Table 1 Library IDs of Instruments and Components in Vessels and Internals

\begin{tabular}{|c|c|c|}
\hline Title / Component Name (if Title is not given) & EDMS ID & Alternate ID \\
\hline Installation in CRP \#2 & Not Existing & E0392-0009-DX \\
\hline EBR-II ACRDS 2 SPEED GEAR TRAIN LAYOUT & 689675 & E1983-0091-DE \\
\hline INSTALLATION IN CRP \#8 & 693838 & E5190-0304-DE \\
\hline INSTALLATION IN CR POSITION \#6 & 694358 & E5221-0001-DE \\
\hline $\begin{array}{l}\text { INSTALLATION OF INSAT 5C1 CR POSITION NO. } \\
11 \text { (2 SHTS) }\end{array}$ & 695317 & E5224-0001-DF \\
\hline SYSTEM SCHEMATIC ACRDS & 696244 & E5257-0019-DE \\
\hline INNER BLANKET SUB-ASSEMBLY & 692790 & EB-1-25056-E \\
\hline Gripper Drive Motor Assembly & 692798 & EB-1-25079-D \\
\hline Rack Drive Motor Assembly & 692820 & EB-1-25101-D \\
\hline Gear Housing Assembly & 692842 & EB-1-25123-B \\
\hline Guide Tube of Control Rod Drive & 692855 & EB-1-25136-D \\
\hline Rack Drive Housing & 692871 & EB-1-25154-C \\
\hline Gripper and Sensing Shaft Bellows & 692881 & EB-1-25164-D \\
\hline Rack Tube & 692886 & EB-1-25169-C \\
\hline Connecting Rod & 692887 & EB-1-25170-B \\
\hline Gripper and Sensing Data Transducers & 693316 & EB-1-25176-C \\
\hline Nesting Bellows & 693317 & EB-1-25177-C \\
\hline PRIMARY TANK ASSEMBLY & 693319 & EB-1-25179-F \\
\hline EBR-II PRIMARY SYSTEM REACTOR INLET PIPING & 693365 & EB-1-25231-F \\
\hline Thermocouples Inner Vessel & 693380 & EB-1-25260-D \\
\hline
\end{tabular}




\begin{tabular}{|c|c|c|}
\hline OUTER BLANKET SUB-ASSEMBLY & 693382 & EB-1-25264-E \\
\hline Thermocouples Inner Vessel Shell Outside Wall & 693383 & EB-1-25266-D \\
\hline Thermocouples Outer Vessel & 693388 & EB-1-25272-D \\
\hline Safety Rod Drive Beam & 693389 & EB-1-25273-D \\
\hline Mark I Core Type Driver Fuel Assembly & 693392 & EB-1-25276-F \\
\hline Shock Absorber Assembly & 693406 & EB-1-25295-F \\
\hline Safety Rod Drive Unit & 693466 & EB-1-25359-E \\
\hline Safety Rod Drive Lower Assembly & 693902 & EB-1-25381-F \\
\hline Main Shafts of Safety Rod Drive Lower Assembly & 693903 & EB-1-25382-E \\
\hline Shielding Tube & 693906 & EB-1-25385-D \\
\hline Rack & 693907 & EB-1-25386-D \\
\hline Bellows Seal & 693916 & EB-1-25395-D \\
\hline Drive Shafts and Universal Joints & 693925 & EB-1-25407-C \\
\hline Rack Housing Assembly & 693927 & EB-1-25409-D \\
\hline EBR-II NEUTRON SHIELDING & 693934 & EB-1-25416-F \\
\hline $\begin{array}{l}\text { Rack Housing Assembly } \\
\text { Shock Absorber Assembly }\end{array}$ & 693954 & EB-1-25442-E \\
\hline INSTRUMENT PLUG & 694389 & EB-1-25582-D \\
\hline INSTRUMENT PLUG & 694390 & EB-1-25584-D \\
\hline "V" Nozzle Plug & 694439 & EB-1-25653-F \\
\hline Mark IA Control Rod & 694502 & EB-1-25731-F \\
\hline Mark I Safety Rod & 694869 & EB-1-25791-F \\
\hline ASSEMBLY REACTOR VESSEL GRID & 694885 & EB-1-25809-E \\
\hline $\begin{array}{l}\text { J. NOZZLE OUTER PLUG "O" RING SPACER } \\
\text { Fission Counter }\end{array}$ & $\begin{array}{l}\mathbf{6 8 9 6 2 3} \\
694898\end{array}$ & EB-1-25822-E \\
\hline Mark I Blanket Region type driver fuel assembly & 693383 & EB-1-25857-F \\
\hline Latch Mechanisms & 695378 & EB-1-26025-D \\
\hline REACTOR VESSEL SUBASSEMBLY & 695949 & EB-1-26290-E \\
\hline Dummy Core Fuel Rod & 695950 & EB-1-26291-D \\
\hline Gripper and Sensing Data Transducers & 695954 & EB-1-26295-C \\
\hline
\end{tabular}




\begin{tabular}{|c|c|c|}
\hline Scram Mechanism & 696791 & EB-1-26588-D \\
\hline Reactor Vessel Cover Labyrinth Seal & 696827 & EB-1-26625-C \\
\hline Control Position Data Transmitter & 697190 & EB-1-26739-C \\
\hline COVER - REACTOR VESSEL & 697236 & EB-1-26799-F \\
\hline REACTOR VESSEL INTERNALS ASSEMBLY & 697641 & EB-1-26954-E \\
\hline SHIELDING CAN LAYOUT & 698059 & EB-1-27172-F \\
\hline $\begin{array}{l}\text { PRESSURE \& TEMPERATURE INSTRUMENTS - } \\
\text { EBR-II VESSEL }\end{array}$ & 698442 & EB-1-27312-E \\
\hline $\begin{array}{l}\text { EBR-II PRIMARY SYSTEM PUMP MI PRESSURE } \\
\text { SENSING INSTALLATION }\end{array}$ & 691522 & EB-1-27564-D \\
\hline Blanket Region Core & 691934 & EB-1-27689-F \\
\hline Ion Chamber & Not Existing & EB-1-28083-E \\
\hline $\begin{array}{l}\text { REACTOR VESSEL, PRESSURE \& RESISTANCE } \\
\text { THERMOMETER INSTRUMENTATION } \\
\text { INSTALLATION }\end{array}$ & 695094 & EB-1-29015-E \\
\hline $\begin{array}{l}\text { REACTOR OUTLET PIPING INSTRUMENTATION } \\
\text { INSTALLATION }\end{array}$ & 695095 & EB-1-29016-E \\
\hline $\begin{array}{l}\text { REACTOR INLET (PUMP) M2 PIPING } \\
\text { INSTRUMENTATION INSTALLATION }\end{array}$ & 695096 & EB-1-29017-E \\
\hline $\begin{array}{l}\text { REACTOR INLET (PUMP) M-1 PIPING } \\
\text { INSTRUMENTATION INSTALLATION }\end{array}$ & 695097 & EB-1-29018-E \\
\hline $\begin{array}{l}\text { THERMOCOUPLE INSTALLATION - REACTOR } \\
\text { VESSEL LOWER PLENUM }\end{array}$ & 695098 & EB-1-29019-E \\
\hline Thermocouples in Primary Pump & 695099 & EB-1-29020-F \\
\hline $\begin{array}{l}\text { REACTOR VESSEL THERMOCOUPLE } \\
\text { INSTALLATION (0-180 DEGREES) }\end{array}$ & 695100 & EB-1-29021-E \\
\hline $\begin{array}{l}\text { REACTOR VESSEL THERMOCOUPLE } \\
\text { INSTALLATION (180-360 DEGREES) }\end{array}$ & 695101 & EB-1-29022-E \\
\hline $\begin{array}{l}\text { PRIMARY TANK COVER PLUG-NOZZLE- } \\
\text { INSTRUMENT ARRANGEMENT }\end{array}$ & 695128 & EB-1-29049-E \\
\hline $\begin{array}{l}\text { THERMOCOUPLE INSTALLATION - BULK } \\
\text { SODIUM }\end{array}$ & 695155 & EB-1-29079-E \\
\hline Blanket Region Core & 696069 & EB-1-29396-F \\
\hline Inner Blanket Neutron Source & 696112 & EB-1-29444-D \\
\hline Source Rod Lifting Tool & 696131 & EB-1-29463-C \\
\hline
\end{tabular}




\begin{tabular}{|c|c|c|}
\hline Outer Blanket Neutron Source & 696132 & EB-1-29464-D \\
\hline Filtering, Core & 697353 & EB-1-29894-E \\
\hline Inner Blanket Filtering Subassembly & 697365 & EB-1-29906-E \\
\hline Manual Operator Assembly & 697377 & EB-1-29919-D \\
\hline Core Flux Wire & Not Existing & EB-1-33847-F \\
\hline Inner \& Outer Blanket Flux Wire & Not Existing & EB-1-33855-D \\
\hline Dummy Plugged Inner Blanket & Not Existing & EB-1-35392-D \\
\hline Dummy Control Rod & Not Existing & EB-1-35393-D \\
\hline Dummy Safety & Not Existing & EB-1-35394-D \\
\hline Neutron Source Storage Thimble & 698633 & EB-1-38154-D \\
\hline Material Surveillance & 691702 & EB-1-38381-E \\
\hline Mark I Oscillator Rod and Thimble & 692158 & EB-1-38560-D \\
\hline $\begin{array}{l}\text { Stainless Steel Drop Rod } \\
\text { Dummy Control Rod }\end{array}$ & 692544 & EB-1-38685-F \\
\hline Mark II Oscillator Rod and Thimble & Not Existing & EB-1-38743-D \\
\hline Oscillator Drive Assembly & 692654 & EB-1-38798-F \\
\hline Drive Shaft Assembly & 692662 & EB-1-38806-F \\
\hline Oscillator Drive Assembly & Not Existing & EB-1-38859-E \\
\hline $\begin{array}{l}\text { Gripper Drive Assembly } \\
\text { PROPOSED MODIFICATION TO EB-1-38859-F } \\
\text { (LOWER DRIVE ASSY- OSCILL. ROD) }\end{array}$ & $\begin{array}{l}693086 \\
721532\end{array}$ & EB-1-38859-F \\
\hline Bearing Carrier Overload Spring Assembly & 693164 & EB-1-38940-D \\
\hline $\begin{array}{l}\text { Sensing Rod Switch Assembly } \\
\text { Gripper Shaft Switch Assembly } \\
\text { Drive Shaft Obstruction Switch Assembly }\end{array}$ & 693174 & EB-1-38950-D \\
\hline Mark IA \& IB Core Type Driver Fuel Assemblies & 693191 & EB-1-38967-F \\
\hline Oscillator Rod Drive & 693198 & EB-1-38974-E \\
\hline Positioning Monitoring Assembly & 693204 & EB-1-38980-E \\
\hline Mark IA Blanket Region Type Driver Fuel Assembly & 693666 & EB-1-39018-F \\
\hline Mark IB Model IHE Control Rod & 693718 & EB-1-39081-F \\
\hline Inner \& Outer Blanket SRB & 700604 & EB-1-39200-E \\
\hline
\end{tabular}




\begin{tabular}{|c|c|c|}
\hline Mark IIB Oscillator and Thimble Assembly & Not Existing & EB-1-39282-D \\
\hline Mark II Core Type Driver Fuel Assembly & 700740 & EB-1-39338-F \\
\hline Mark I Blanket Region Type Driver Fuel Subassembly & 701022 & EB-1-39348-F \\
\hline Mark I Core Type Driver Fuel Subassembly & 701025 & EB-1-39351-F \\
\hline Mark IIB Oscillator and Thimble Assembly & Not Existing & EB-1-39475-F \\
\hline Fuel Rod used in Place of Mark II Oscillator Rod & 701155 & EB-1-39495-F \\
\hline Non-Fueled Dummy Oscillator Rod & 701591 & EB-1-39690-D \\
\hline Top Spacer of Model IHC Control assembly & 701592 & EB-1-39691-C \\
\hline Higher Worth Control Rod & 701850 & EB-1-39914-F \\
\hline Revised Drop Rod, Model RRR & 702959 & EB-1-50516-F \\
\hline Tantalum Drop Rod, Model TAD & 703885 & EB-1-50854-F \\
\hline Biological Shield & Not Existing & $\begin{array}{l}\text { H.K. Ferguson Drwg. } \\
\text { No. R-12 } \sim \text { R-22 }\end{array}$ \\
\hline Outlet Air duct from Thimbles J-2, J-3, O-1, O-2 & Not Existing & HKF-R-300 \\
\hline Outlet Air duct from Thimbles J-1, J-4, O-3, O-4 & Not Existing & HKF-R-303 \\
\hline Outer Blanket Instrument Tube & Not Existing & RE-1-33695-D \\
\hline Inner Blanket Instrument Tube & Not Existing & RE-1-33703-D \\
\hline Core Thermocouples & Not Existing & RE-1-33776-D \\
\hline Inner Blanket Fission Foil Traverse & Not Existing & RE-1-33790-D \\
\hline Outer Blanket Fission Foil Traverse & Not Existing & RE-1-34025-D \\
\hline Dry Critical Oscillator Rod & Not Existing & RE-1-34361-E \\
\hline Core Filter & Not Existing & RE-1-35395-D \\
\hline Outer Blanket Filter & Not Existing & RE-1-35396-D \\
\hline
\end{tabular}

Table 2 Library IDs for Instrument in Primary Loop

\begin{tabular}{|c|c|c|}
\hline Title & EDMS ID & Alternate ID \\
\hline $\begin{array}{l}\text { NO. } 1 \text { NUCLIDE TRAP ASSY PRIMARY } \\
\text { PURIFICATION CELL }\end{array}$ & 697485 & E5171-0003-DE \\
\hline $\begin{array}{l}\text { EBR-II PRIMARY TANK SUPPORT } \\
\text { STRUCTURE } \\
\text { EBR II UPPER SUPPORT STRUCTURE } \\
\text { PARTS (ORIENTATION) }\end{array}$ & $\begin{array}{l}693347 \\
693349\end{array}$ & EB-1-25208-F \\
\hline
\end{tabular}




\begin{tabular}{|l|l|l|}
\hline Primary Auxiliary Pump & 693356 & EB-1-25219-F \\
\hline Thermocouples at Secondary Sodium Outlet Pipe & 693373 & EB-1-25250-C \\
\hline Intermediate Heat Exchanger & 693376 & EB-1-25256-F \\
\hline D-C Electromagnetic Pump & 693452 & EB-1-25344-E \\
\hline $\begin{array}{l}\text { REACTOR OUTLET PIPING } \\
\text { INSTRUMENTATION INSTALLATION }\end{array}$ & $\mathbf{6 9 5 0 9 5}$ & EB-1-29016-E \\
\hline Piping within Primary Tank & 696880 & EB-1-29655-D \\
\hline Surge Tank & 697319 & EB-1-29858-C \\
\hline Thermocouples at Bottom Orifice Plate & Not Existing & $\begin{array}{l}\text { Struthers-Wells Corp. Drwg. } \\
59 P 8088 D 3\end{array}$ \\
\hline Thermocouples at Top Orifice Plate & Not Existing & $\begin{array}{l}\text { Struthers-Wells Corp. Drwg. } \\
59 P 8088 D 4\end{array}$ \\
\hline Tube Bundle & Not Existing & $\begin{array}{l}\text { Struthers-Wells Corp. Drwg. } \\
\text { 59P8088D6 }\end{array}$ \\
\hline Well Casing & Not Existing & $\begin{array}{l}\text { Struthers-Wells Corp. Drwg. } \\
\text { 59P8088D7 }\end{array}$ \\
\hline Shield Plug & Not Existing & $\begin{array}{l}\text { Struthers-Wells Corp. Drwg. } \\
\text { 59P8088D9 }\end{array}$ \\
\hline Thermocouples at Primary Sodium Outlet & $\begin{array}{l}\text { Struthers-Wells Corp. Drwg. } \\
59 P 8088 D 17\end{array}$ \\
\hline
\end{tabular}

\section{Locations of Sensors in EBR-II System 4.1 Identification Codes of Sensors}

The location of sensors in the plant layout is shown in Figure 1. This figure, located in pp.6.1-7/8, Chapter 6, Volume II, shows the sketchy location of sensors in the plant. The instrumentations in the primary cooling system are shown in Figure 2. Each sensor and the associated signal are identified by its location, system, parameter, function, instrument number and point of measurement. How to read this identification code is described in EBR-II System Design Descriptions, Volume II, Chapter 6, section 6.3.3.1 Instruments. This document have been made available as part of this work as an electronic pdf file.

The structure of the code, the possible values of the code entries, theirs meaning are here reported in the following tables.

Table 3 Identification Code Structure [Vol. II, Chapter 6, P.6.3-5]

\begin{tabular}{|l|l|}
\hline Position & Information content \\
\hline 1 & $\begin{array}{l}\text { Location (relative to building or section of plant where instrument is being } \\
\text { located). One character. }\end{array}$ \\
\hline 2 & System. Two digits integer. \\
\hline
\end{tabular}




\begin{tabular}{|l|l|}
\hline 3 & Parameter. One or two characters. \\
\hline 4 & Function. One character. \\
\hline 5 & Instrument Number. 3 digits Number \\
\hline 6 & Points of Measurement \\
\hline
\end{tabular}

For instance, Multi-point temperature indicator with alarm in steam system, located in the power plant is P3-TIA-503-24. This identification code is generated as following:

Table 4 Identification Code for Location

\begin{tabular}{|l|l|}
\hline \multicolumn{2}{|l|}{ Location [Vol. II, Chapter 6, P.6.3-6] } \\
\hline B & Boiler Plant - Wing \\
\hline C & Cooling Tower \\
\hline E & Electrical Substation \\
\hline F & Process Plant \\
\hline G & Guard House \\
\hline K & Ambulance and Fire Station \\
\hline L & Laboratory Building \\
\hline M & Pump House and Well No.1 \\
\hline N & Pump House and Well No.2 \\
\hline P & Power Plant (including auxiliary boilers) \\
\hline R & Reactor Plant \\
\hline S & Sodium Plant - Wing \\
\hline T & Temporary Facilities \\
\hline W & Waste Treatment Plant \\
\hline Y & $\begin{array}{l}\text { Yard and Services (including fuel oil pumping station and main oil } \\
\text { storage tank) }\end{array}$ \\
\hline
\end{tabular}


Table 5 Identification Code for System

\begin{tabular}{|l|l|}
\hline \multicolumn{2}{|l|}{ System [Vol. II, Chapter 6, P.6.3-6] } \\
\hline 1 & Primary System \\
\hline 2 & Secondary System \\
\hline 3 & Steam Power System \\
\hline 4 & Shutdown Cooling \\
\hline 5 & Feedwater System \\
\hline 6 & Argon \\
\hline 7 & Cooling Tower \\
\hline 8 & Shield Air Cooling \\
\hline 9 & Steam Heating System \\
\hline 10 & Electrical \\
\hline 11 & Suspect and Contaminated Gas \\
\hline 12 & Waste Disposal \\
\hline 13 & General Ventilation \\
\hline 14 & Air Conditioning \\
\hline 15 & Portable and Fire Water Systems \\
\hline 16 & Fuel Oil System \\
\hline 17 & Space Air Cooling \\
\hline 18 & Water Treatment \\
\hline
\end{tabular}

Table 6 Identification Code for Parameter

\begin{tabular}{|l|l|}
\hline \multicolumn{2}{|l|}{ Parameter [Vol. II, Chapter 6, P.6.3-7] } \\
\hline N & Nuclear \\
\hline T & Temperature \\
\hline F & Flow \\
\hline P & Pressure \\
\hline L & Level \\
\hline M & Radiation \\
\hline pH & pH \\
\hline Q & Current \\
\hline
\end{tabular}




\begin{tabular}{|l|l|}
\hline V & Voltage \\
\hline KW & Power \\
\hline Va. & Volt. Amp. \\
\hline dP & Differential Pressure \\
\hline dT & Differential Temperature \\
\hline C & Conductivity \\
\hline B & Position \\
\hline Y & Leak Detector \\
\hline RH & Relative Humidity \\
\hline S & Speed \\
\hline G & Chlorine Concentration \\
\hline X & Miscellaneous \\
\hline A & Annunciator \\
\hline
\end{tabular}

Table 7 Identification Code for Function

\begin{tabular}{|l|l|}
\hline \multicolumn{2}{|l|}{ Function [Vol. II, Chapter 6, P.6.3-7] } \\
\hline R & Record \\
\hline C & Control \\
\hline I & Indicate \\
\hline A & Alarm (or electrical contact) \\
\hline S & Integrate (subletter) \\
\hline Z & Analytical \\
\hline D & Data Logging (only) \\
\hline
\end{tabular}

Table 8 Identification Code for Component

\begin{tabular}{|l|l|}
\hline \multicolumn{2}{|l|}{ Component [Vol. II, Chapter 6, P.6.3-8] } \\
\hline Q & Nuclear Detector \\
\hline TC & Thermocouple \\
\hline RT & Resistance Thermometer \\
\hline TT & Temperature Transmitter (pneumatic) \\
\hline PT & Pressure Cell \\
\hline
\end{tabular}




\begin{tabular}{|l|l|}
\hline LT & Level Transmitter \\
\hline AM & Amplifier \\
\hline pHT & pH Cell \\
\hline VT & Potential Trans. \\
\hline QT & Current Trans. \\
\hline E & Primary Element (dead ended) \\
\hline FT & Flow Transmitter (except magnetic) \\
\hline VC & Control Valve (pneumatic, hydraulic or electrical) \\
\hline VS & Solenoid Valve \\
\hline PS & Pressure Switch \\
\hline KS & Power Supply \\
\hline X & Miscellaneous \\
\hline FM & Flow Transmitter (magnetic) \\
\hline SD & Sodium Detector \\
\hline LD & Leak Detector \\
\hline
\end{tabular}




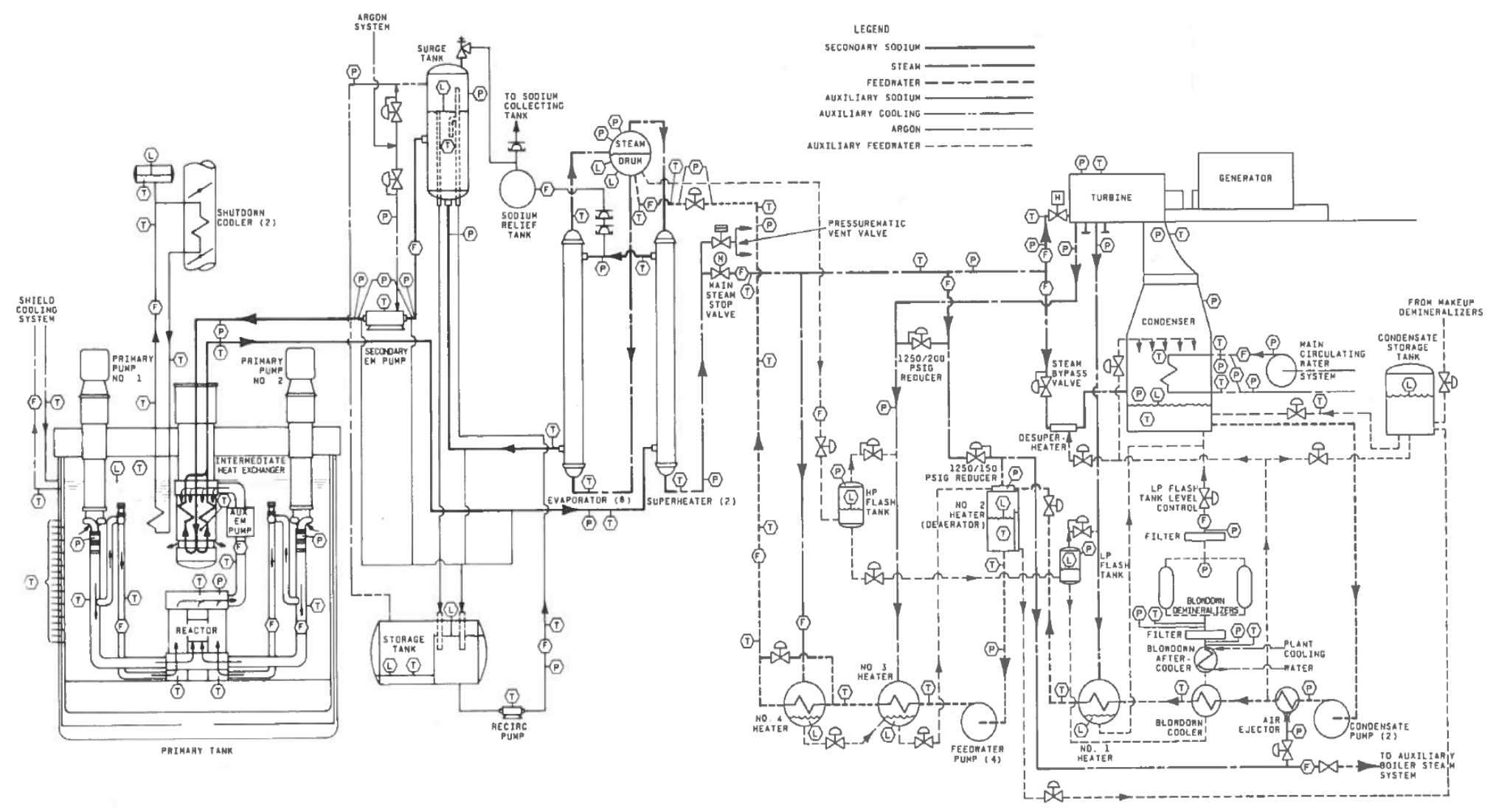

Figure 1 Figure 6.1-1 EBR-II Flow Diagram [Vol. II, Chapter 6, pp.6.1-7/8] 


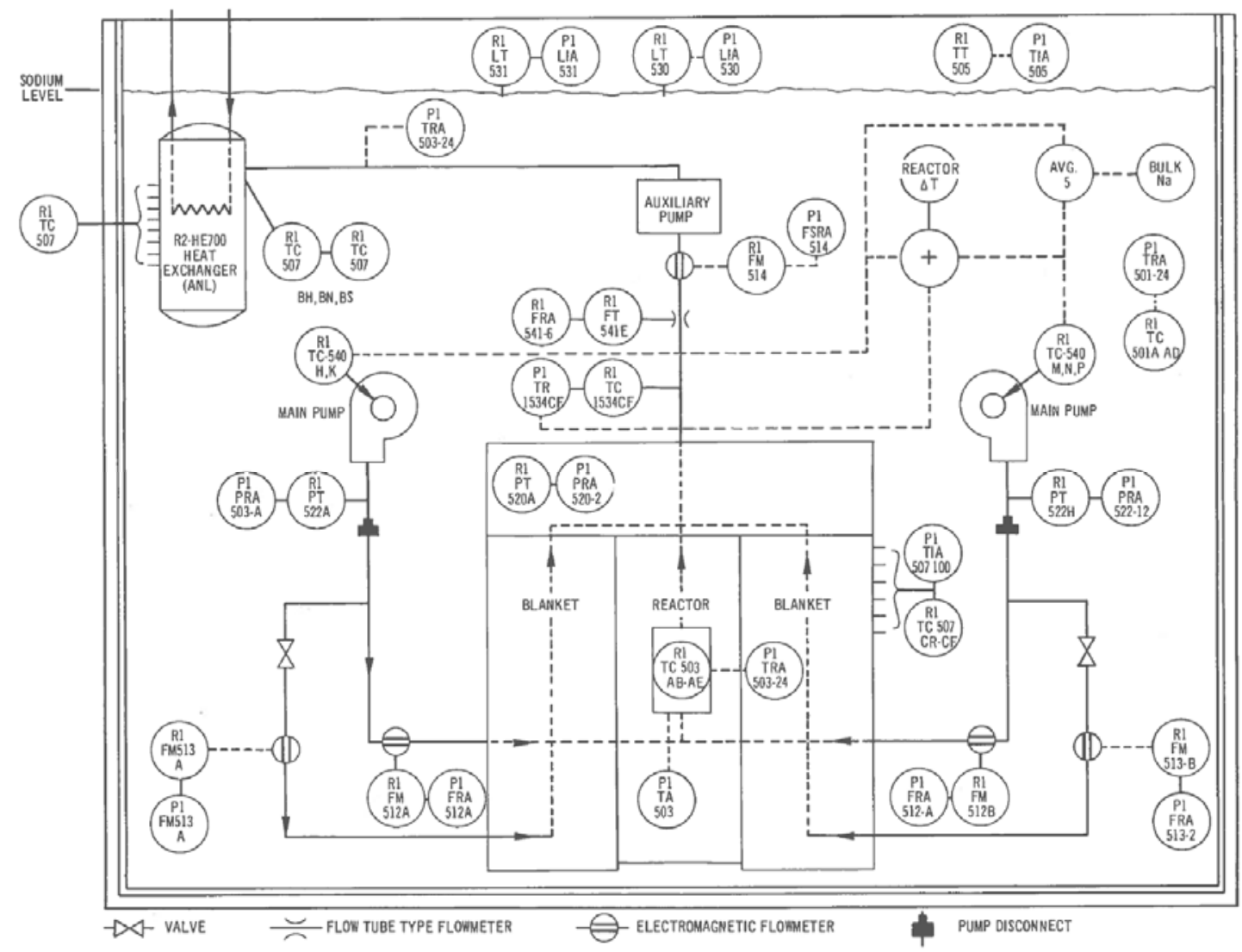

Figure 2 Figure 6.3-25 Primary Cooling System Instrumentation [Vol. II, Chapter 6, p.6.3-57] 


\subsection{Lists and Locations of Sensors}

The nuclear instrument thimbles are installed into the reactor core to perform various experiments and to detect the physical conditions of the core. Figure 3 shows the top view of the primary tank and the locations of the nuclear instrument thimbles. The detailed information of sensors in each nozzle is reported in Table 9. Drawings of nuclear instrument thimbles and thimble cooling is shown in Figure 4. The type and location of instrument shown in Figure 4 are listed in Table 10. The specific drawings of "O", "J-1", J-2", "J-3" and "J-4" thimbles are shown in Figure 5, Figure 6 and Figure 7, respectively.

Instrument locations in the reactor vessel are shown in Figure 8. In addition, the location and description of each instrument are tabulated in Table 11 and Table 12. The instrument locations in the reactor vessel and the neutron shield are shown in Figure 9 and corresponding information of those instruments is tabulated in Table 13.

Schematic diagrams of primary tanks No.1 and 2 are shown in Figure 10 and Figure 11, respectively. In these figures, the types and locations of the sensors are presented. The type, location and number of each instrument in primary tank No. 1 and No. 2 are tabulated in Table 14 and Table 15, respectively.

Figure 12 shows the bulk sodium and Argon sensors in primary tank. The detailed locations of the sensors in Figure 12 are tabulated in Table 16.

Locations of thermocouples on the primary tank are shown in Figure 13. Totally 137 thermocouples are installed in the primary tank. The location and instrument number of the sensors are tabulated in Table 17.

Figure 14 shows the cross-sectional views of intermediate heat exchanger (IHX) and locations of the thermocouples in the IHX. The detailed information of the thermocouples in Figure 14 is tabulated in Table 18.

The instruments in shutdown coolers are presented in Figure 15. The locations of the instrument in shutdown coolers are tabulated in Table 19. 


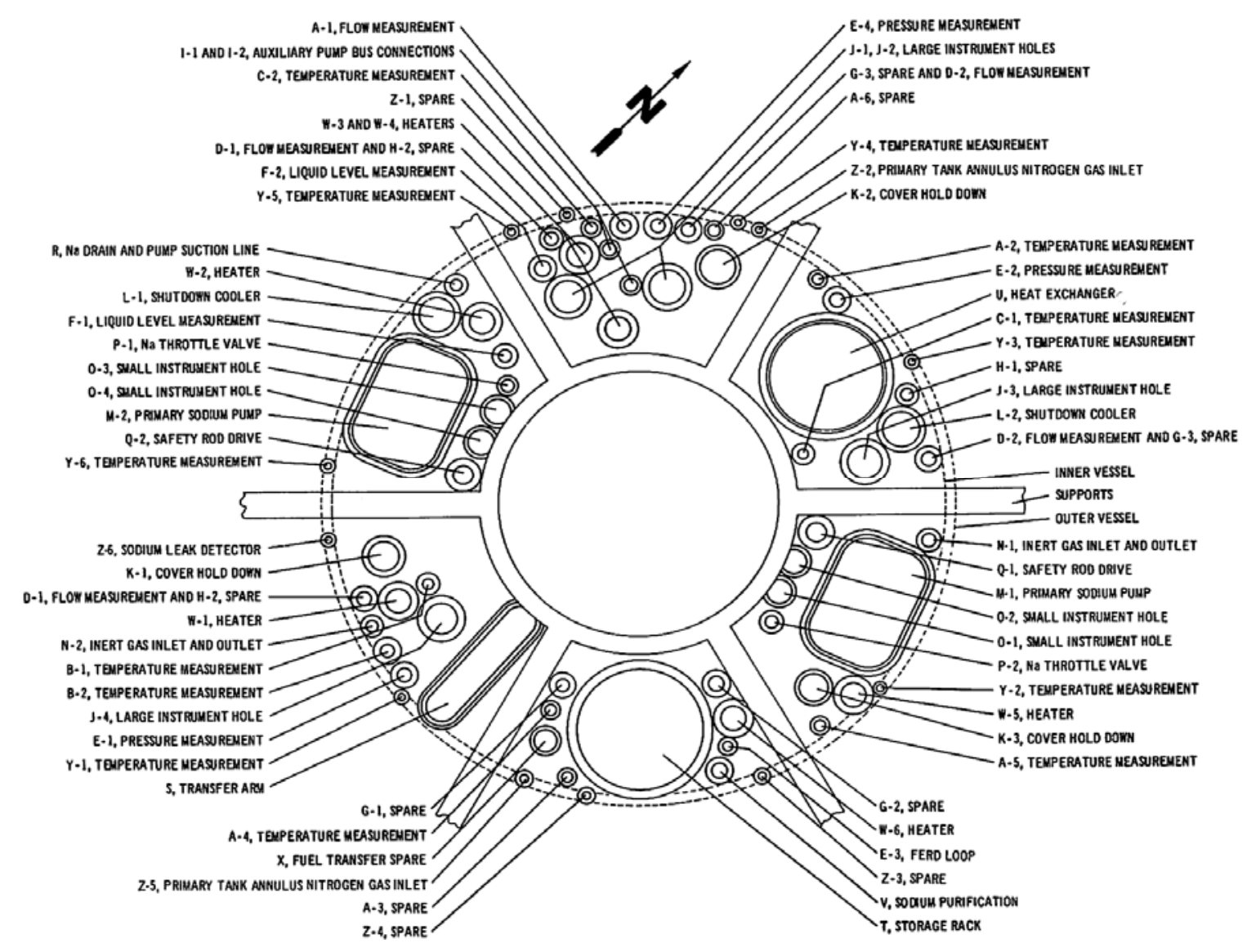

Figure 3 Figure 4.6-5 Primary Tank Nozzles (or Figure 6.3-24 Instrument Lead Penetration through Primary Tank Cover) [Vol. II, Chapter 4, p.4.6-11 or Vol. II, Chapter 6, pp.6.3-55/56] 
Table 9 Figure 4.6-5 Primary Tank Nozzles (Figure 6.3-24 Instrument Lead Penetration through Primary Tank Cover) [Vol. II, Chapter 4, p.4.6-11 or Vol. II, Chapter 6, pp.6.3-55/56]

\begin{tabular}{|c|c|c|c|c|c|c|c|c|}
\hline Nozzle & $\begin{array}{c}\text { Instruments } \\
\text { R1- }\end{array}$ & $\begin{array}{c}\text { Layout Dwg. } \\
\text { EB-1- }\end{array}$ & Nozzle & $\begin{array}{c}\text { Instruments } \\
\text { R1- }\end{array}$ & $\begin{array}{c}\text { Layout Dwg. } \\
\text { EB-1- }\end{array}$ & Nozzle & $\begin{array}{c}\text { Instruments } \\
\text { R1- }\end{array}$ & $\begin{array}{c}\text { Layout Dwg. } \\
\text { EB-1- }\end{array}$ \\
\hline \multirow[t]{6}{*}{ A-1 } & RT-502-A & 29015-E & \multirow[t]{19}{*}{$\mathrm{C}-1$} & RT-504A & $29020-\mathrm{F}$ & $\mathrm{F}-1$ & LT-531 & 29049-E \\
\hline & RT-502B & 29015-E & & RT-504B & $29020-\mathrm{F}$ & $\mathrm{F}-2$ & LT-530 & 29049-E \\
\hline & RT-506 & 29016-E & & TC-507CX & 29022-E & \multirow[t]{6}{*}{ Y-1 } & TC-507A & 25260-D \\
\hline & RT-506X & \multirow[b]{3}{*}{$\downarrow$} & & TC-507CY & \multirow{8}{*}{$\frac{\gamma}{29020-\mathrm{F}}$} & & TC-507B & \\
\hline & FM-514 & & & TC-507CZ & & & TC-507C & \\
\hline & FT-541E & & & TC-507DF & & & TC-507D & \\
\hline \multirow[t]{13}{*}{ A-2 } & TC-530 & \multirow[t]{11}{*}{$29020-\mathrm{F}$} & & TC-507DG & & & TC-507E & \\
\hline & TC-540Q & & & TC-507DH & & & TC-507F & \\
\hline & TC-540R & & & TC-507DP & & \multirow[t]{10}{*}{$\mathrm{Y}-2$} & TC-507G & 25266-D \\
\hline & TC-540S & & & TC-507DT & & & TC-507H & \\
\hline & TC-540T & & & TC-507AB & & & TC-507K & \\
\hline & TC-540V & & & TC-507AC & & & TC-507M & \\
\hline & TC-540W & & & TC-507AD & & & TC-507N & \\
\hline & TC-540X & & & TC-507AE & & & TC-507P & \\
\hline & TC-540Y & & & TC-507AM & & & TC-507Q & \\
\hline & TC-540Z & & & TC-507AN & & & TC-507R & \\
\hline & TC-540AA & & & TC-507AP & & & TC-507S & \\
\hline & TC-1534CF & 29016-E & & TC-507AQ & 7 & & $\mathrm{TC}-507 \mathrm{~T}$ & \\
\hline & TC-1534CG & 29016-E & & TC-507AT & 29019-E & \multirow[t]{10}{*}{$\mathrm{Y}-3$} & TC-507V & 25266-D \\
\hline \multirow[t]{6}{*}{ A-4 } & TC-540A & $29020-\mathrm{F}$ & \multirow[t]{18}{*}{$\mathrm{C}-2$} & TC-501R & 29079-E & & TC-507W & \\
\hline & TC-540B & & & TC-501S & & & TC-507X & \\
\hline & TC-540C & & & TC-501T & & & TC-507Y & \\
\hline & TC-540D & & & TC-501V & & & TC-507Z & \\
\hline & TC-540E & & & TC-501W & & & TC-507AA & \\
\hline & TC-540F & & & TC-501X & & & TC-507AB & \\
\hline \multirow[t]{6}{*}{ A-5 } & TC-540G & $29020-\mathrm{F}$ & & TC-501Z & & & TC-507AC & \\
\hline & TC-540H & & & TC-501AB & & & TC-507AD & \\
\hline & TC-540K & & & TC-501AC & & & $\mathrm{TC}-507 \mathrm{AE}$ & \\
\hline & TC-540AR & & & TC-507CV & 29021-E & $\mathrm{Y}-4$ & TC-507AF & 25260-D \\
\hline & TC-540AS & & & TC-507CW & & & TC-507AG & \\
\hline & TC-540AV & $\downarrow$ & & TC-507DD & & & TC-507AH & \\
\hline \multirow[t]{16}{*}{ B-1 } & TC-501A & 29079-E & & TC-507DE & & & TC-507AK & \\
\hline & TC-501B & & & TC-507DN & & & TC-507AM & \\
\hline & TC-501C & & & TC-507DS & & & TC-507AN & \\
\hline & TC-501D & & & TC-540M & 29020-E & Y-5 & TC-507AP & 25266-D \\
\hline & TC-501E & & & TC-540N & & & TC-507AQ & \\
\hline & TC-501F & & & TC-40P & & & TC-507AR & \\
\hline & TC-501G & & $\mathrm{H}-2$ & FM-512B & 29017-E & & TC-507AS & \\
\hline & TC-501H & & & FM-513B & & & TC-507AT & \\
\hline & TC-501K & & & FT-541B & & & TC-507AV & \\
\hline & TC-501M & & & FT-541D & $\downarrow$ & & TC-507AW & \\
\hline & TC-501N & & G-3 & FM-512A & 29018-E & & TC-507AX & \\
\hline & TC-501P & & & FM-513A & & & TC-507AY & \\
\hline & TC-501Q & & & FT-541A & & Y-6 & TC-507AZ & 25266-D \\
\hline & TC-501Y & & & FT-541C & 7 & & TC-507BA & \\
\hline & TC-501AA & & E-1 & PT-540A & 29015-E & & TC-507BB & \\
\hline & TC-501AD & & & PT-522E & 29017-E & & TC-507BC & \\
\hline B-2 & TC-507CR & 29021-E & & PT-522F & & & TC-507BD & \\
\hline & TC-507CS & & & PT-522G & & & TC-507BE & \\
\hline & TC-507CT & & & PT-522Q & 7 & & TC-507BF & \\
\hline & TC-507DA & & E-2 & PT-522B & 29018-E & & TC-507BG & \\
\hline & TC-507DB & & & PT-522C & & & TC-507BH & \\
\hline & TC-507DC & & & PT-522D & & & TC-507FE & \\
\hline & TC-507DK & & & PT-522K & $\downarrow$ & M-1 & PT-522A & 27564-D \\
\hline & TC-507DM & & & PT-522P & 29015-E & M-2 & PT-522H & 27564-D \\
\hline & TC-507DQ & & E-4 & TC-505 & 29015-E & & & \\
\hline & TC-507DR & & & PT-520B & & & & \\
\hline & TC-507FG & 29079-E & & PT-522M & 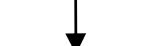 & & & \\
\hline & TC-507FH & & & PT-522N & & & & \\
\hline & TC-507FK & $\nabla$ & & PT-522R & & & & \\
\hline & & & & PT-522S & & & & \\
\hline
\end{tabular}



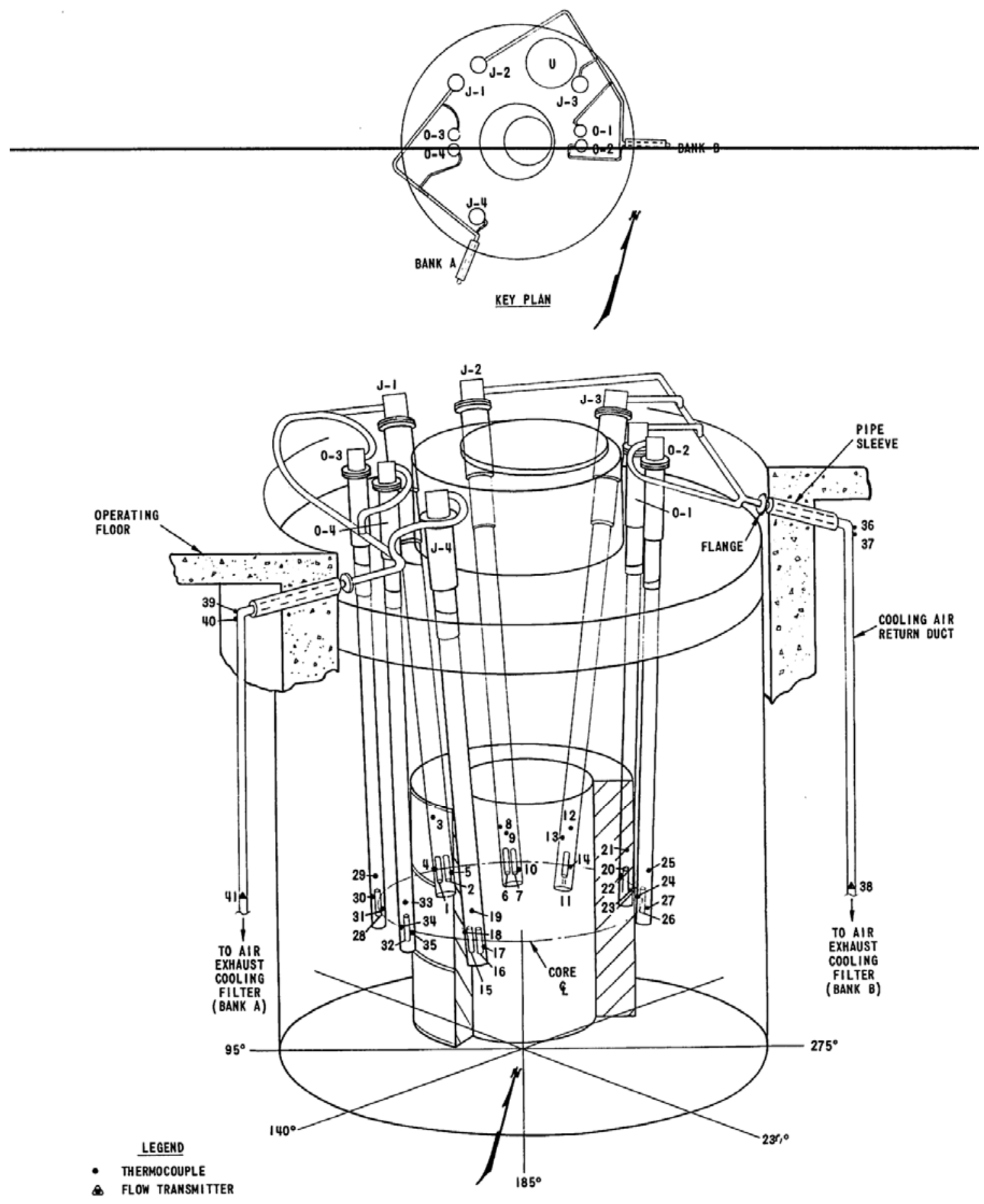

Figure 4 Figure 6.3-1 Nuclear Instrument Thimbles and Thimble Cooling [Vol. II, Chapter 6, pp.6.313/14] 
Table 10 Figure 6.3-1 Nuclear Instrument Thimbles and Thimble Cooling [Vol. II, Chapter6, pp.6.3-13/14]

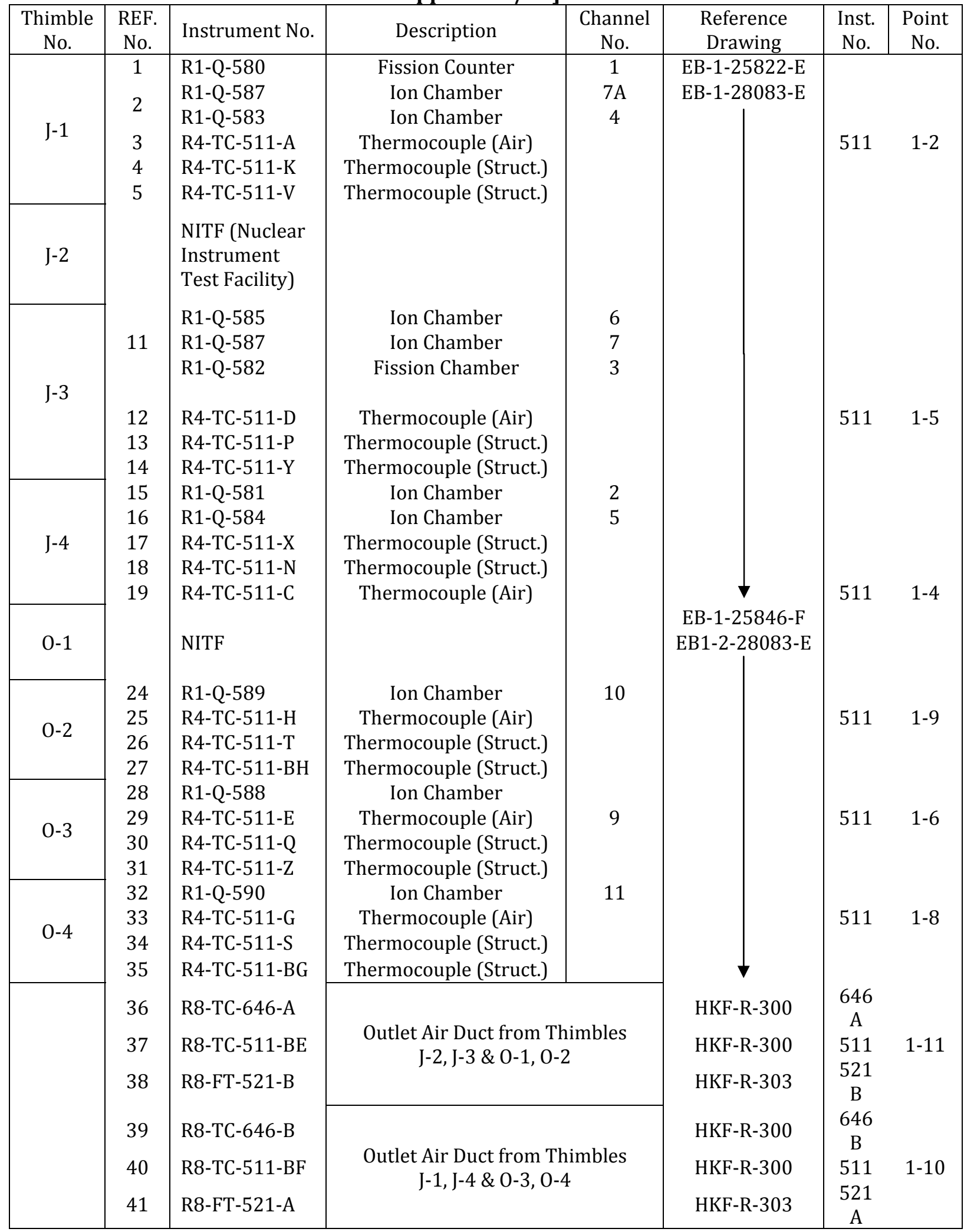




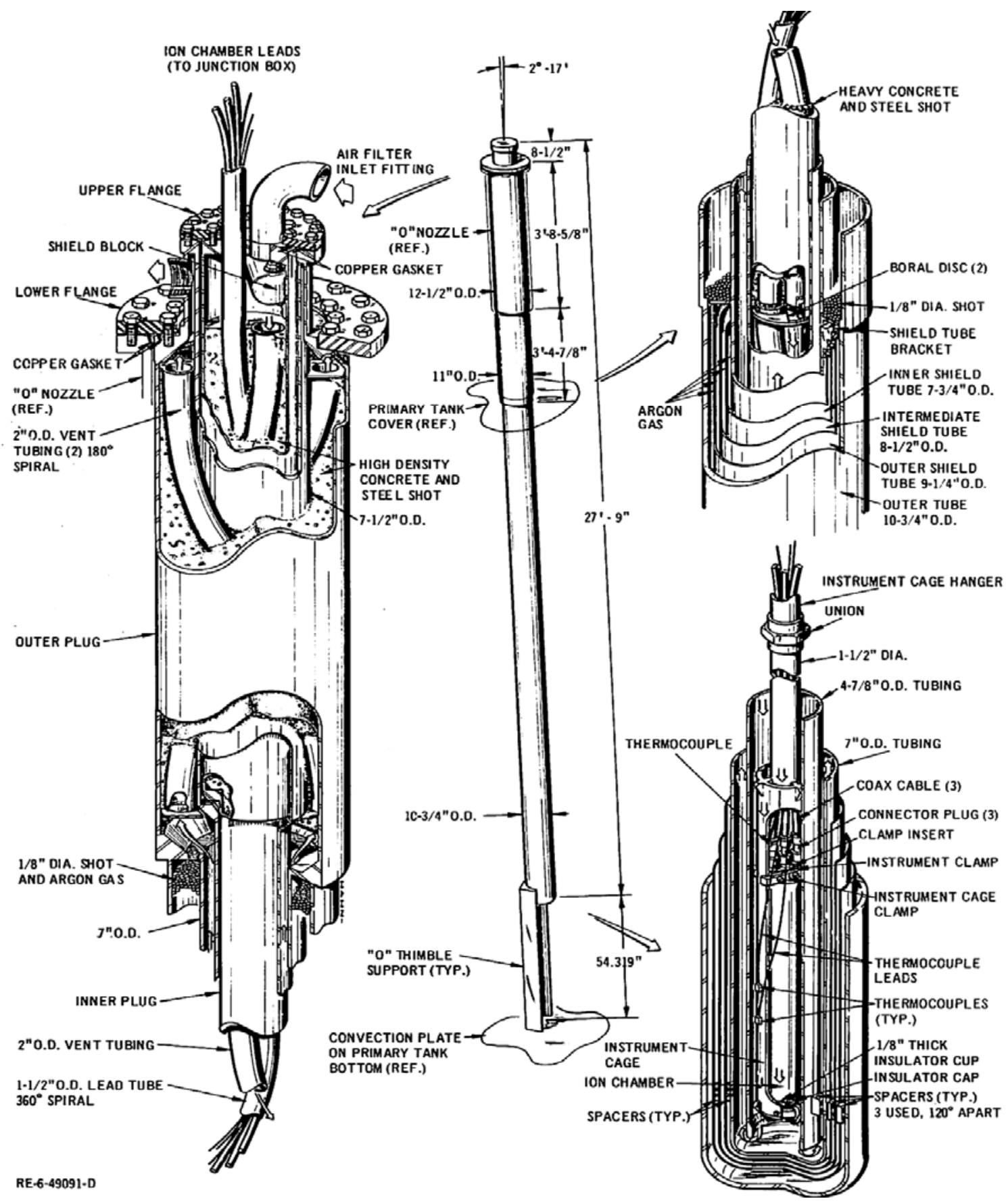

Figure 5 Figure 7.4-5 “O” Instrument Thimble Assembly (showing Original Instrumentation Arrangement) [Vol. II, Chapter 7, p.7.4-11] 


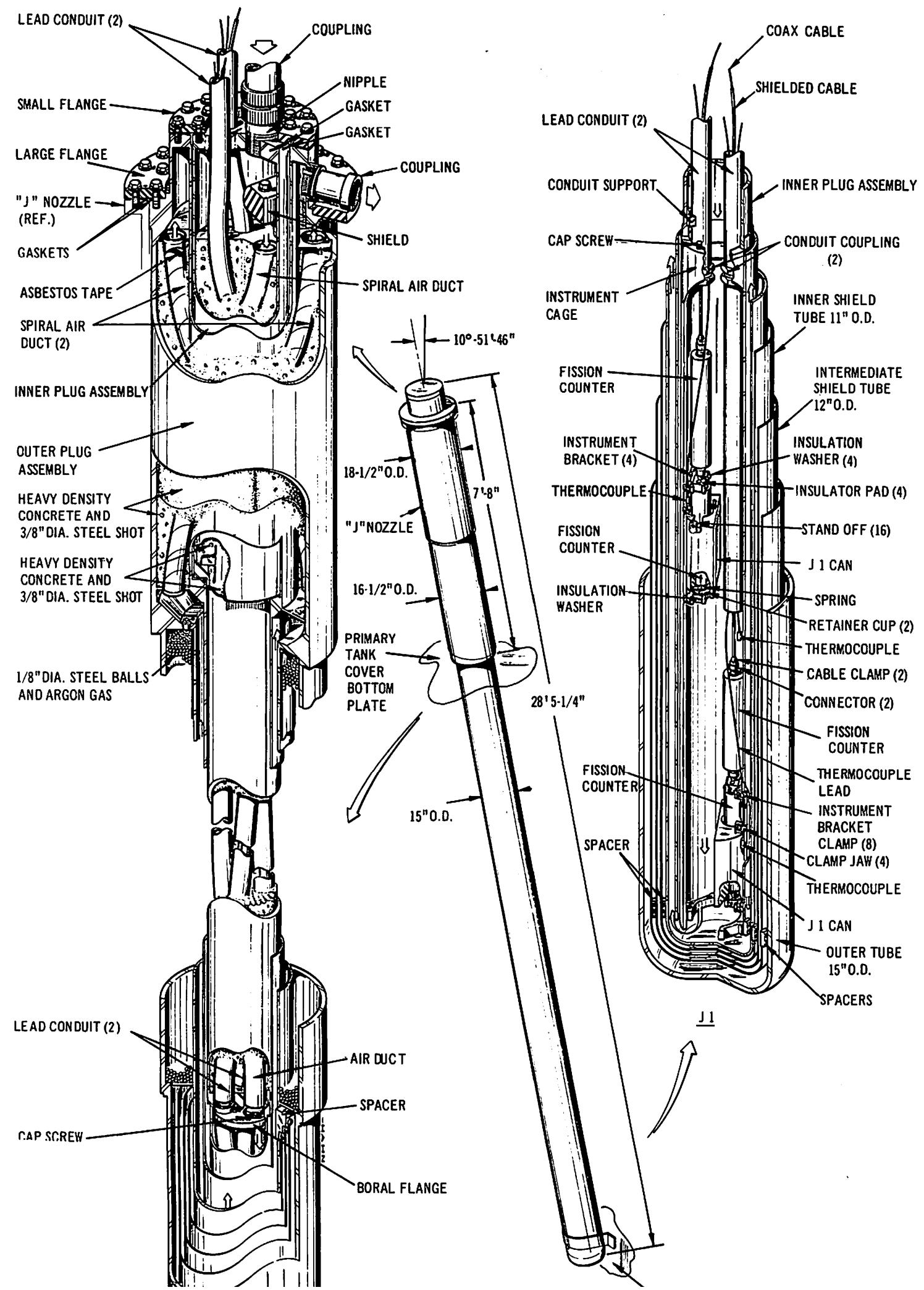

Figure 6 Figure 7.4-6 "J-1" Instrument Thimble Assembly (showing Original Instrumentation Arrangement) [Vol. II, Chapter 7, p.7.4-14] 


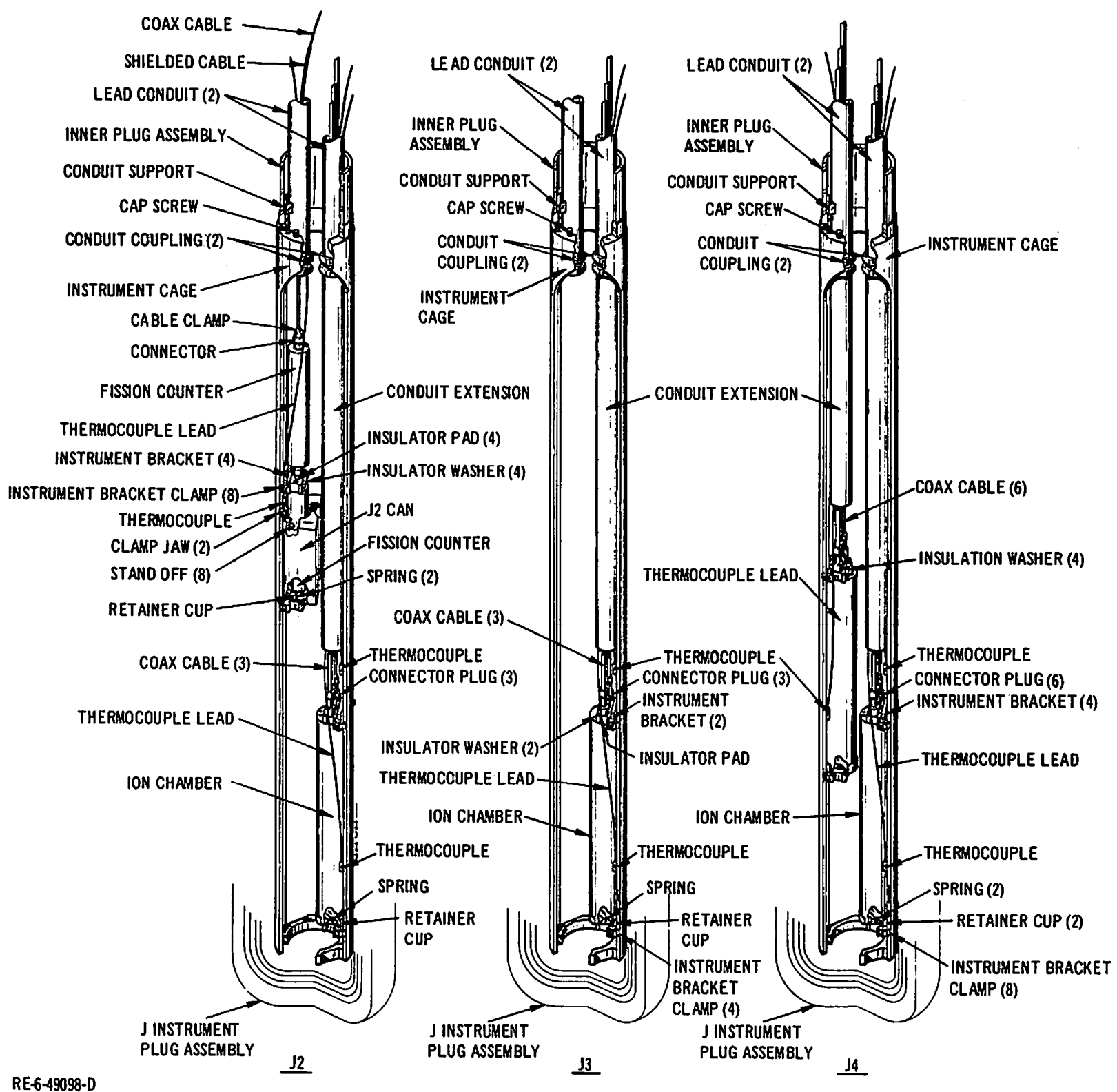

Figure 7 Figure 7.4-7 Original Instrument Cage Area Arrangement J-2, J-3 and J-4 Thimbles [Vol. II, Chapter 7, p.7.4-16] 


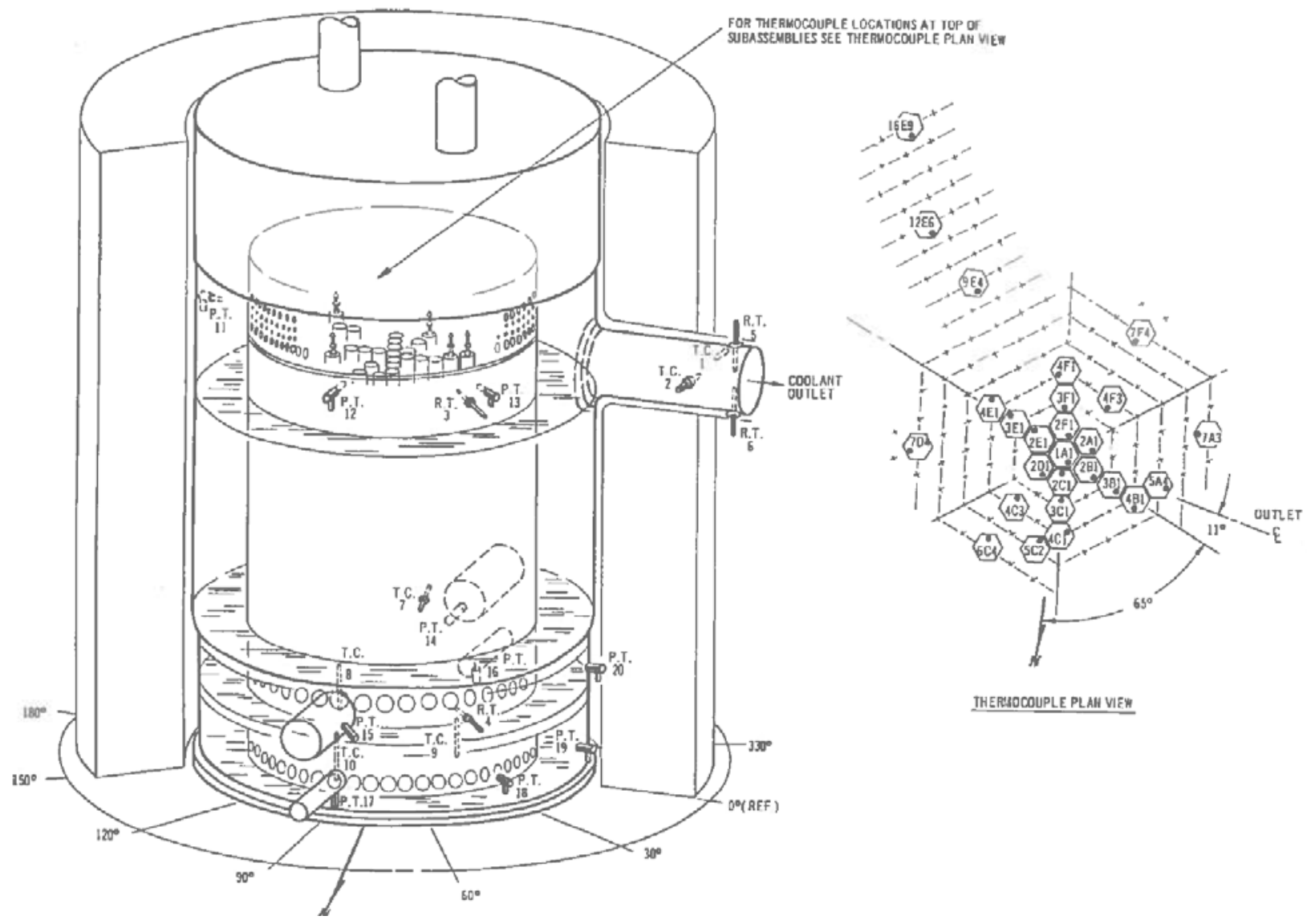

Figure 8 Figure 6.3-5 Instrument Locations in Reactor Vessel [Vol. II, Chapter 6, pp.6.3-19/20] 
Table 11 Figure 6.3-5 Instrument Locations in Reactor Vessel [Vol. II, Chapter 6, pp. 6.3-19/20]

\begin{tabular}{|c|c|c|c|c|}
\hline $\begin{array}{l}\text { Ref. } \\
\text { No. }\end{array}$ & Location & Instrument No. & $\begin{array}{l}\text { Reference } \\
\text { Drawing }\end{array}$ & Description \\
\hline 1 & Na Outlet Pipe Jacket & $\begin{array}{l}\text { R1-TC-1534- } \\
\text { CG }\end{array}$ & EB-1-29016-E & Thermocouple \\
\hline 2 & Reactor Coolant Outlet & $\begin{array}{l}\text { R1-TC-1534- } \\
\text { CF }\end{array}$ & EB-1-29016-E & Thermocouple \\
\hline 3 & Reactor Coolant Outlet Annular Plenum & R1-RT-502-B & EB-1-29015-E & Res. Thermo. \\
\hline 4 & Reactor Coolant High Press. Annular Plenum & R1-RT-502-A & EB-1-29015-E & \\
\hline 5 & Reactor Coolant Outlet & R1-RT-506 & EB-1-29016-E & \\
\hline 6 & Reactor Coolant Outlet & R1-RT-506X & EB-1-29016-E & $\downarrow$ \\
\hline 7 & Reactor Coolant High Press. Annular Plenum & R1-TC-540-At & EB-1-29016-E & Thermocouple \\
\hline 8 & Reactor Coolant Low Press. Plenum & R1-TC-540-AV & EB-1-29016-E & \\
\hline 9 & Reactor Coolant Low Press. Plenum & R1-TC-540-AR & EB-1-29016-E & \\
\hline 10 & Reactor Coolant Low Press. Plenum & R1-TC-540-AS & EB-1-29016-E & 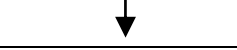 \\
\hline 11 & Reactor Coolant Outlet Annular Plenum & R1-PT-520-A & EB-1-27312-E & Press. Transm. \\
\hline 12 & Reactor Coolant Outlet Annular Plenum & R1-PT-520-B & EB-1-27312-E & \\
\hline 13 & Reactor Coolant Outlet Annular Plenum & R1-PT-522-R & EB-1-27312-E & \\
\hline 14 & Reactor Vessel High Press. Inlet Pipe & R1-PT-522-F & EB-1-25231-F & \\
\hline 15 & Reactor Vessel High Press. Inlet Pipe & R1-PT-522-C & EB-1-25231-F & \\
\hline 16 & Reactor Vessel Low Press. Inlet Pipe & R1-PT-522-E & EB-1-25231-F & \\
\hline 17 & Reactor Vessel Low Press. Inlet Pipe & R1-PT-522-D & EB-1-25231-F & \\
\hline 18 & Reactor Coolant Low Press. Annular Plenum & R1-PT-522-S & EB-1-27312-E & \\
\hline 19 & Reactor Coolant Low Press. Annular Plenum & R1-PT-522-M & EB-1-27312-E & \\
\hline 20 & Reactor Coolant High Press. Annular Plenum & R1-PT-522-N & EB-1-27312-E & $\nabla$ \\
\hline
\end{tabular}

Table 12 Figure 6.3-5 Instrument Locations in Reactor Vessel [Vol. II, Chapter 6, pp. 6.3-19/20]

\begin{tabular}{|c|l|}
\hline Ref. No & \multicolumn{1}{|c|}{ Instrument No. } \\
\hline 1A1 & R1-TC-503-AA \\
\hline 2C1 & R1-TC-503-D \\
\hline 2D1 & R1-TC-503-A \\
\hline $2 \mathrm{E} 1$ & R1-TC-503-P \\
\hline $2 \mathrm{~F} 1$ & R1-TC-503-K \\
\hline $2 \mathrm{~A} 1$ & R1-TC-503-H \\
\hline 2B1 & R1-TC-503-E \\
\hline 3C1 & R1-TC-503-C \\
\hline 3E1 & R1-TC-503-M \\
\hline
\end{tabular}

\begin{tabular}{|c|l|}
\hline Ref. No & \multicolumn{1}{|c|}{ Instrument No. } \\
\hline 3F1 & R1-TC-503-T \\
\hline 3B1 & R1-TC-503-F \\
\hline 4C1 & R1-TC-503-B \\
\hline 4C3 & R1-TC-503-AC \\
\hline 4E1 & R1-TC-503-Z \\
\hline 4F1 & R1-TC-503-V \\
\hline 4F3 & R1-TC-503-S \\
\hline 4B1 & R1-TC-503-G \\
\hline 7D4 & R1-TC-503-AD \\
\hline
\end{tabular}

\begin{tabular}{|c|l|}
\hline Ref. No & \multicolumn{1}{|c|}{ Instrument No. } \\
\hline $5 A 4$ & R1-TC-503-Q \\
\hline 5C2 & R1-TC-503-Y \\
\hline 6C4 & R1-TC-503-N \\
\hline 7F4 & R1-TC-503-AE \\
\hline 7A3 & R1-TC-503-AB \\
\hline 9E4 & R1-TC-503-R \\
\hline 12E6 & R1-TC-503-X \\
\hline 16E9 & R1-TC-503-W \\
\hline & \\
\hline
\end{tabular}




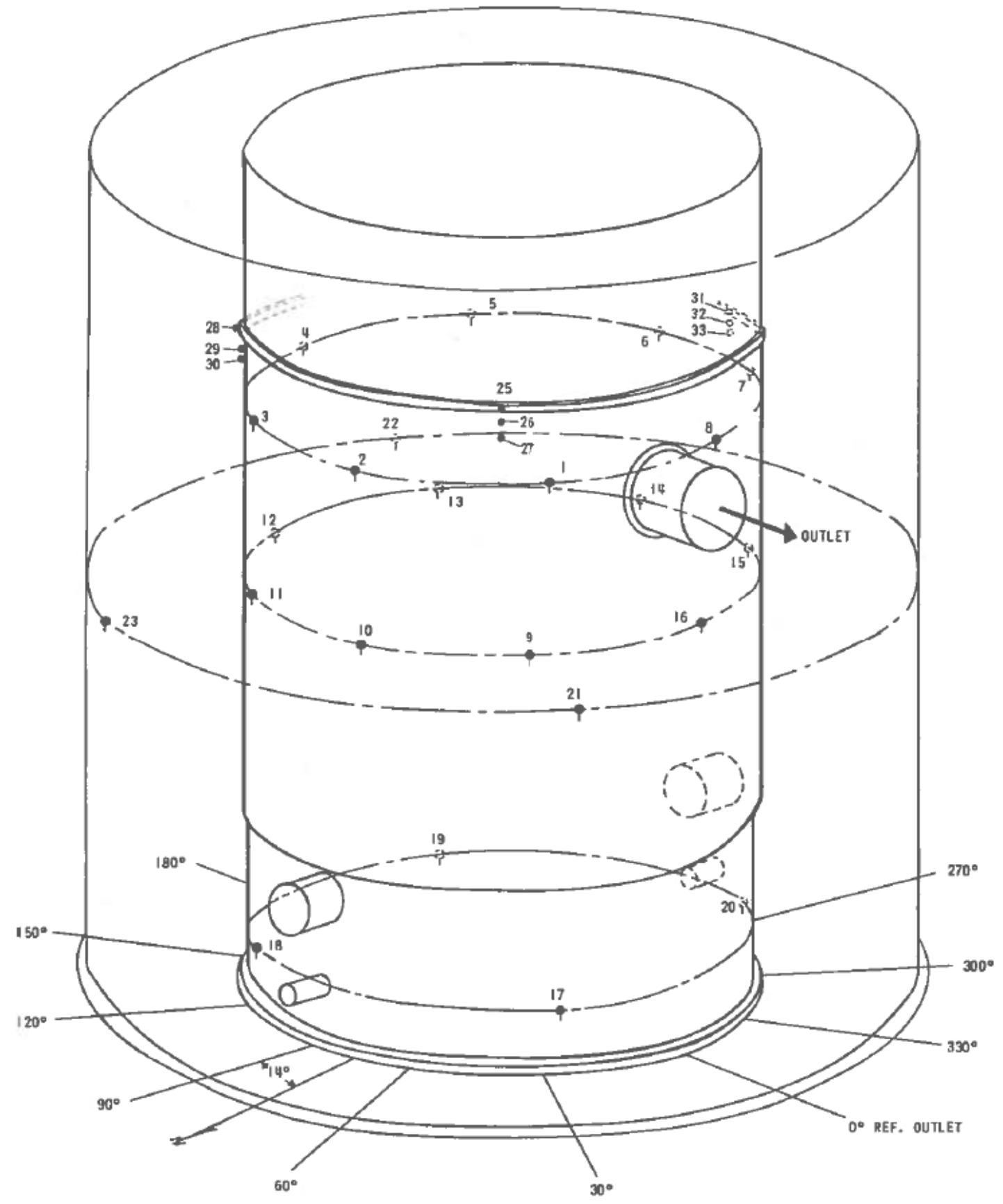

Figure 9 Figure 6.3-6 Reactor Vessel and Neutron Shield [Vol. II, Chapter 6, pp.6.3-21/22] 
Table 13 Figure 6.3-6 Reactor Vessel and Neutron Shield [Vol. II, Chapter 6, pp.6.3-21/22]

\begin{tabular}{|c|c|c|c|c|}
\hline $\begin{array}{l}\text { Ref. } \\
\text { No. }\end{array}$ & Location & Instrument No. & Reference Drawing & $\begin{array}{c}\text { Point No. } \\
\text { P1-TRA-507 }\end{array}$ \\
\hline $1^{*}$ & Reactor Vessel Outside & R1-TC-507-DE & EB-1-29021-E & 89 \\
\hline 2 & Wall & R1-TC-507-DF & EB-1-29022-E & 90 \\
\hline $3^{*}$ & & R1-TC-507-DG & EB-1-29022-E & 91 \\
\hline 4 & & R1-TC-507-DH & EB-1-29022-E & 92 \\
\hline $5^{*}$ & & R1-TC-507-DA & EB-1-29021-E & 85 \\
\hline 6 & & R1-TC-507-DB & EB-1-29021-E & 86 \\
\hline $7^{*}$ & & R1-TC-507-DC & EB-1-29021-E & 87 \\
\hline 8 & & R1-TC-507-DD & EB-1-29021-E & 88 \\
\hline $9 *$ & & R1-TC-507-CW & EB-1-29021-E & 81 \\
\hline 10 & & R1-TC-507-CX & EB-1-29022-E & 82 \\
\hline $11^{*}$ & & R1-TC-507-CY & EB-1-29022-E & 83 \\
\hline 12 & & R1-TC-507-CZ & EB-1-29022-E & 84 \\
\hline $13^{*}$ & & R1-TC-507-CR & EB-1-29021-E & 77 \\
\hline 14 & & R1-TC-507-CS & EB-1-29021-E & 78 \\
\hline $15^{*}$ & & R1-TC-507-CT & EB-1-29021-E & 79 \\
\hline 16 & & R1-TC-507-CV & EB-1-29021-E & 80 \\
\hline $17^{*}$ & & R1-TC-507-DN & EB-1-29021-E & 95 \\
\hline 18 & & R1-TC-507-DP & EB-1-29022-E & 96 \\
\hline $19 *$ & & R1-TC-507-DK & EB-1-29021-E & 93 \\
\hline 20 & & R1-TC-507-DM & EB-1-29021-E & 94 \\
\hline 21 & Reactor Vessel Neutron & R1-TC-507-DS & EB-1-29021-E & 99 \\
\hline 22 & Shield Outer Wall & R1-TC-507-DQ & EB-1-29022-E & 97 \\
\hline 23 & & R1-TC-507-DT & EB-1-29021-E & 100 \\
\hline 24 & $\nabla$ & & & \\
\hline 25 & *Reactor Vessel Outside & R1-TC-540XX3 & EB-1-29021-E & 18 \\
\hline 26 & Wall Top Ring & R1-TC-540XX1 & EB-1-29021-E & 16 \\
\hline 27 & & R1-TC-540XX2 & EB-1-29021-E & 17 \\
\hline 28 & & R1-TC-540XX6 & EB-1-29021-E & 21 \\
\hline 29 & & R1-TC-540XX4 & EB-1-29021-E & 19 \\
\hline 30 & & R1-TC-540XX5 & EB-1-29021-E & 20 \\
\hline 31 & & R1-TC-540XX9 & EB-1-29022-E & 24 \\
\hline 32 & & R1-TC-540XX7 & EB-1-29022-E & 22 \\
\hline 33 & $\downarrow$ & R1-TC-540XX8 & EB-1-29022-E & 23 \\
\hline
\end{tabular}

* ON RX-T1-1535 


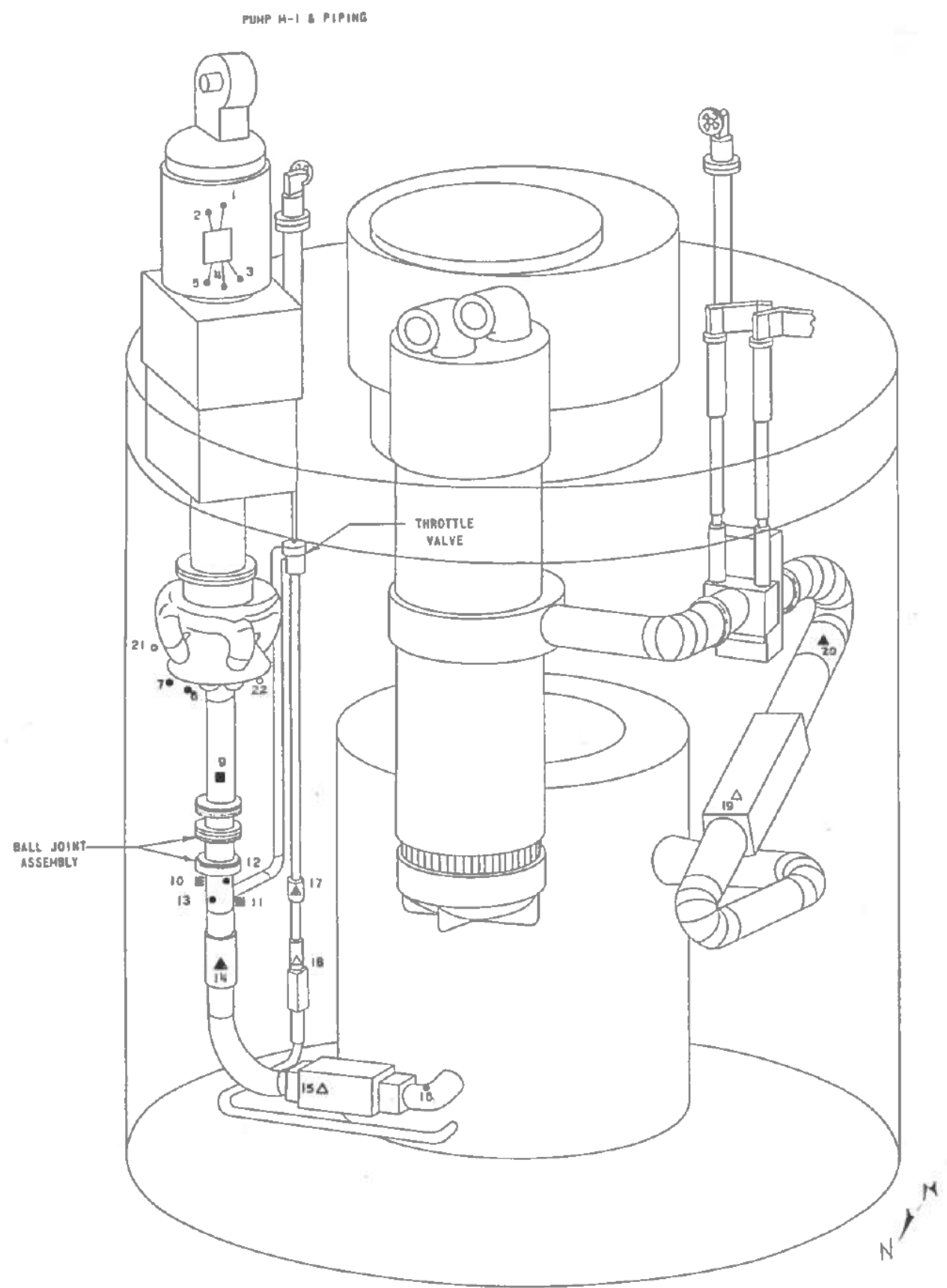

Figure 10 Figure 6.3-7 Primary Pump No.1 [Vol. II, Chapter 6, pp.6.3-23/24] 
Table 14 Figure 6.3-7 Primary Pump No.1 [Vol. II, Chapter 6, pp.6.3-23/24]

\begin{tabular}{|c|c|c|c|c|c|}
\hline $\begin{array}{l}\text { Ref. } \\
\text { No. }\end{array}$ & $\begin{array}{l}\text { Instrument } \\
\text { No. }\end{array}$ & Readout Inst. & $\begin{array}{c}\text { Point } \\
\text { No. }\end{array}$ & Location & Ref. Dwg. No. \\
\hline 1 & & Pump \#1 Control Panel & 11 & Pump Motor upper Bearing & \multirow{5}{*}{$\begin{array}{c}\text { BYRON } \\
\text { JACKSON 2E- } \\
1571\end{array}$} \\
\hline 2 & & Pump \#1 Control Panel & 3 & Pump Motor Winding & \\
\hline 3 & & Pump \#1 Control Panel & 9 & Pump Flange & \\
\hline 4 & & Pump \#1 Control Panel & 10 & Pump Motor Lower Bearing & \\
\hline 5 & & Pump \#1 Control Panel & 4 & Pump Motor Air Outlet & \\
\hline 6 & R1-TC-540-H & 540 & 8 & Pump Inlet & EB-1-29020-F \\
\hline 7 & R1-TC-540-K & 540 & 9 & Pump Inlet & EB-1-29020-F \\
\hline 8 & DELETED & & & & \\
\hline 9 & R1-PT-522-A & 522 & 1 & Pump Outlet Before Disc. Joint & EB-1-27564-D \\
\hline 10 & R1-PT-522-B & 522 & 2 & Pump Outlet After Disc. Joint & EB-1-29018-E \\
\hline 11 & R1-PT-522-P & SPARE & & Pump Outlet After Disc. Joint & EB-1-29018-E \\
\hline 12 & R1-TC-540-C & 540 & 3 & Pump Outlet After Disc. Joint & EB-1-29020-F \\
\hline 13 & R1-TC-540-D & 540 & 4 & Pump Outlet After Disc. Joint & EB-1-29020-F \\
\hline 14 & R1-FT-541-C & 541 & 2 & Pump Outlet High Press. Pipe & EB-1-29018-E \\
\hline 15 & R1-FM-512-A & $512 \mathrm{~A}$ & -- & Pump Outlet High Press. Pipe & EB-1-29018-E \\
\hline 16 & R1-TC-540-B & 540 & 1 & Pump Outlet High Press. Pipe & EB-1-29020-F \\
\hline 17 & R1-FT-541-A & 541 & 1 & Pump Outlet Low Press. Pipe & EB-1-29018-E \\
\hline 18 & R1-FM-513-A & 513 & 1 & Pump Outlet Low Press. Pipe & EB-1-29018-E \\
\hline 19 & R1-FM-514 & P1-FsRA-514 & & Reactor Outlet Pipe & EB-1-29016-E \\
\hline 20 & R1-FT-541-E & R1-FRA-541 & 5 & Reactor Outlet Pipe & EB-1-29016-E \\
\hline 21 & R1-RT-504-D & 504 & -- & Pump Inlet & -- \\
\hline 22 & R1-RT-504-B & 504 & -- & Pump Inlet & -- \\
\hline 0 & & \multicolumn{4}{|l|}{ Resistance Thermometer } \\
\hline & & \multicolumn{4}{|l|}{ Thermocouple } \\
\hline & & \multicolumn{4}{|l|}{ Pressure Sensor } \\
\hline $\bar{\Delta}$ & & \multicolumn{4}{|l|}{ EM Flow Meter } \\
\hline$\vec{\Delta}$ & & \multicolumn{4}{|c|}{ Foster Flowmeter (Flow Tube) } \\
\hline
\end{tabular}




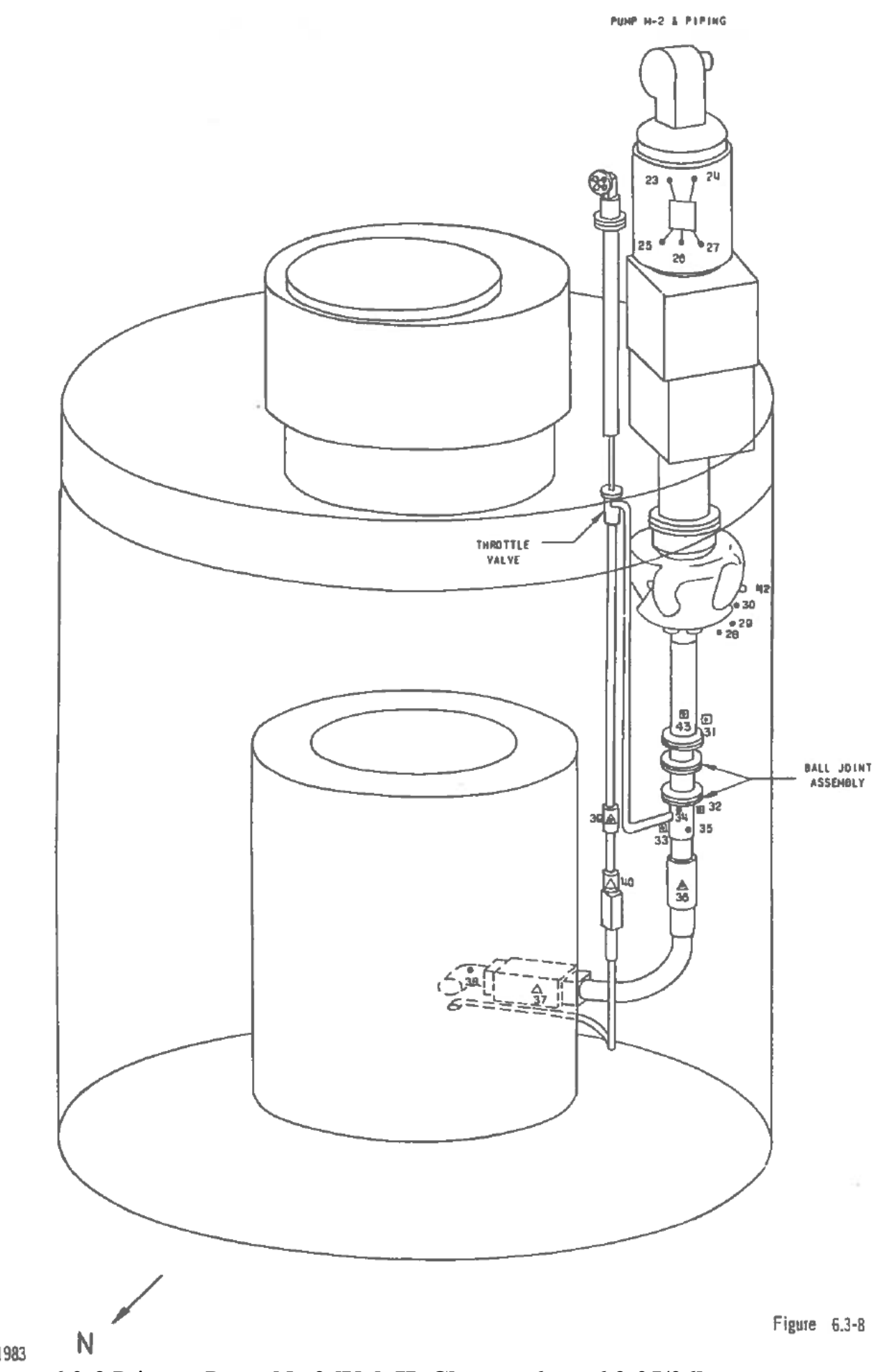

Figure 11 Figure 6.3-8 Primary Pump No.2 [Vol. II, Chapter 6, pp.6.3-25/26] 
Table 15 Figure 6.3-8 Primary Pump No.2 [Vol. II, Chapter 6, pp.6.3-25/26]

\begin{tabular}{|c|c|c|c|c|c|}
\hline $\begin{array}{l}\text { Ref. } \\
\text { No. }\end{array}$ & $\begin{array}{l}\text { Instrument } \\
\text { No. }\end{array}$ & Readout Inst. & $\begin{array}{l}\text { Point } \\
\text { No. }\end{array}$ & Location & Ref. Dwg. No. \\
\hline 23 & & Pump \#2 Control Panel & 11 & Pump Motor upper Bearing & \multirow{5}{*}{$\begin{array}{l}\text { BYRON } \\
\text { JACKSON } \\
2 \mathrm{E}-1571\end{array}$} \\
\hline 24 & & Pump \#2 Control Panel & 3 & Pump Motor Winding & \\
\hline 25 & & Pump \#2 Control Panel & 9 & Pump Flange & \\
\hline 26 & & Pump \#2 Control Panel & 10 & Pump Motor Lower Bearing & \\
\hline 27 & & Pump \#2 Control Panel & 4 & Pump Motor Air Outlet & \\
\hline 28 & R1-TC-540-M & 540 & 10 & Pump Inlet & EB-1-29020-F \\
\hline 29 & R1-TC-540-N & 540 & 11 & Pump Inlet & EB-1-29020-F \\
\hline 30 & R1-TC-540-P & 540 & 12 & Pump Inlet & EB-1-29020-F \\
\hline 31 & R1-PT-522-H & 522 & 8 & $\begin{array}{l}\text { Pump Outlet Before Disc. } \\
\text { Joint }\end{array}$ & EB-1-27564-D \\
\hline 32 & R1-PT-522-G & 522 & 7 & Pump Outlet After Disc. Joint & EB-1-29017-E \\
\hline 33 & R1-PT-522-0 & SPARE & & Pump Outlet After Disc. Joint & EB-1-29017-E \\
\hline 34 & R1-TC-540-F & 540 & 6 & Pump Outlet After Disc. Joint & EB-1-29020-F \\
\hline 35 & R1-TC-540-E & 540 & 5 & Pump Outlet After Disc. Joint & EB-1-29020-F \\
\hline 36 & R1-FT-541-D & 541 & 4 & $\begin{array}{l}\text { Pump Outlet High Press. } \\
\text { Pipe }\end{array}$ & EB-1-29017-E \\
\hline 37 & R1-FM-512-B & 512B & - & $\begin{array}{l}\text { Pump Outlet High Press. } \\
\text { Pipe }\end{array}$ & EB-1-29017-E \\
\hline 38 & R1-TC-540-A & 540 & 2 & $\begin{array}{l}\text { Pump Outlet High Press. } \\
\text { Pipe }\end{array}$ & EB-1-29020-F \\
\hline 39 & R1-FT-541-B & 541 & 2 & Pump Outlet Low Press. Pipe & EB-1-29020-F \\
\hline 40 & R1-FM-513-B & 513 & 2 & Pump Outlet Low Press. Pipe & EB-1-29020-F \\
\hline 41 & DELETED & -- & -- & & -- \\
\hline 42 & R1-RT-504-E & 504 & -- & Pump Inlet & -- \\
\hline 43 & $\begin{array}{l}\text { R1-PT-522- } \\
\text { HX }\end{array}$ & DAS & & $\begin{array}{l}\text { Pump Outlet Before Disc. } \\
\text { Joint }\end{array}$ & $\begin{array}{l}\text { 1982-0118- } \\
\text { ED-00 }\end{array}$ \\
\hline \multirow{5}{*}{\multicolumn{2}{|c|}{0}} & \multicolumn{4}{|l|}{ Resistance Thermometer } \\
\hline & & \multicolumn{4}{|l|}{ Thermocouple } \\
\hline & & \multicolumn{4}{|l|}{ Pressure Sensor } \\
\hline & & \multicolumn{4}{|l|}{ EM Flow Meter } \\
\hline & & \multicolumn{4}{|c|}{ Foster Flowmeter (Flow Tube) } \\
\hline
\end{tabular}




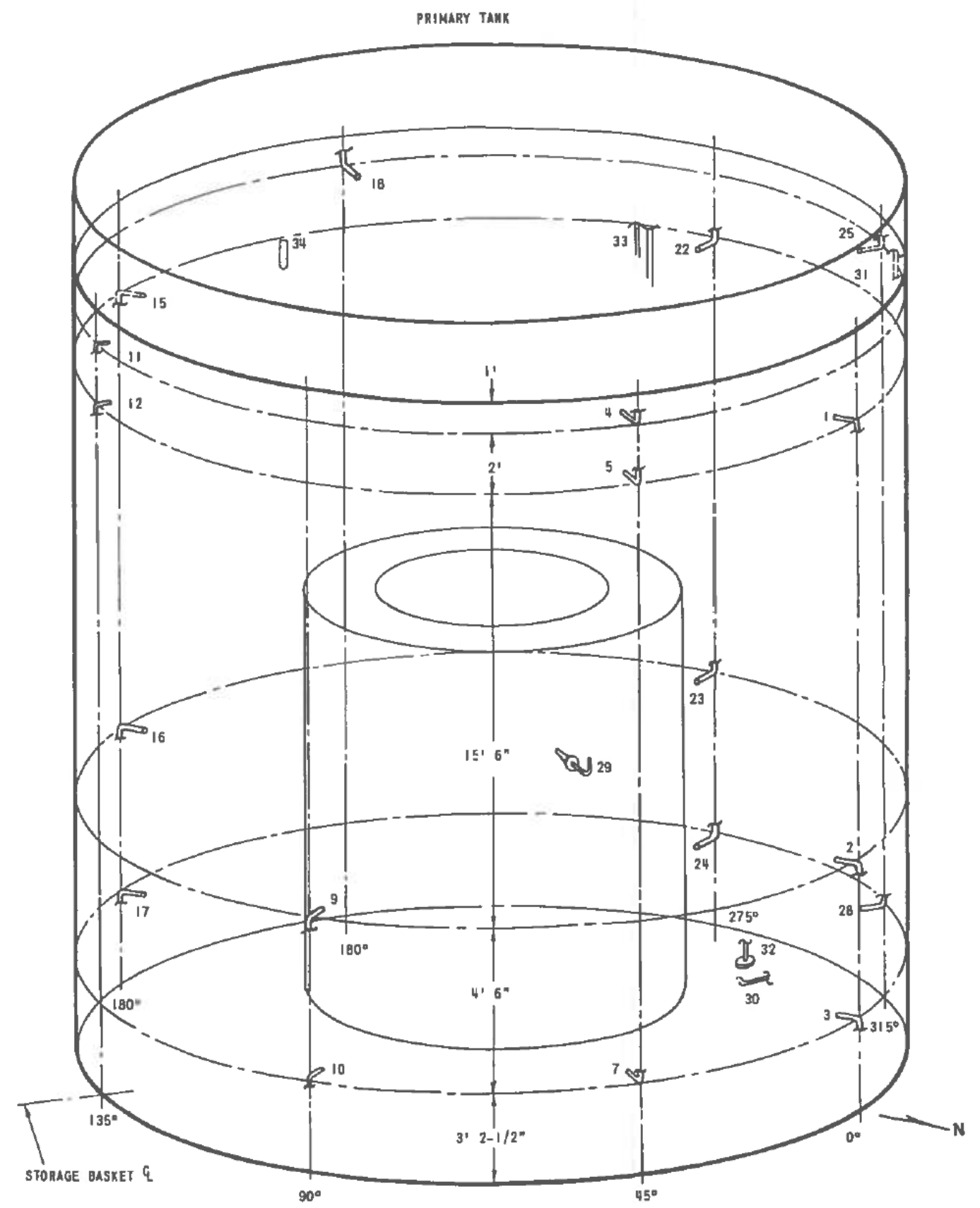

Figure 12 Figure 6.3-9 Bulk Sodium and Argon Sensors in Primary Tank [Vol. II, Chapter 6, pp.6.327/28] 
Table 16 Figure 6.3-9 Bulk Sodium and Argon Sensors in Primary Tank [Vol. II, Chapter 6, pp.6.327/28]

\begin{tabular}{|c|c|c|c|c|}
\hline $\begin{array}{l}\text { Ref. } \\
\text { No. }\end{array}$ & Location & Instrument No. & $\begin{array}{c}\text { Reference } \\
\text { Drawing }\end{array}$ & $\begin{array}{c}\text { Point } \\
\text { P1-TRA-501 }\end{array}$ \\
\hline 1 & Bulk Sodium & R1-TC-501-T & $\begin{array}{l}\text { EB1-2-29079- } \\
\text { E }\end{array}$ & \\
\hline 2 & Bulk Sodium & R1-TC-501-V & & \\
\hline 3 & Bulk Sodium & R1-TC-501-W & & \\
\hline 4 & Argon Blanket (Spare) & R1-TC-501-EX & & \\
\hline 5 & Argon Blanket (Spare) & R1-TC-501-X & & 18 \\
\hline 7 & Argon Blanket (Spare) & R1-TC-501-Z & & 21 \\
\hline 9 & Bulk Sodium & R1-TC-501-H & & 8 \\
\hline 10 & Bulk Sodium & R1-TC-501-Q & & 13 \\
\hline 11 & $\begin{array}{l}\text { Argon Blanket in Scram Circuit } \\
\text { (Gas Blanket Scram) }\end{array}$ & R1-TC-501-Y & & \\
\hline 12 & Bulk Sodium & R1-TC-501-AA & & \\
\hline 15 & Bulk Sodium & R1-TC-501-D & & 4 \\
\hline 16 & Bulk Sodium & R1-TC-501-E & & 5 \\
\hline 17 & Bulk Sodium & R1-TC-501-F & & 6 \\
\hline 18 & Argon Blanket (Spare) & R1-TC-501-FG & & \\
\hline 22 & Argon Blanket (Spare) & R1-TC-501-M & & \\
\hline 23 & Bulk Sodium & R1-TC-501-N & & 16 \\
\hline 24 & Bulk Sodium & R1-TC-501-P & & 11 \\
\hline 25 & Argon Blanket (Spare) & R1-TC-501-FH & & 12 \\
\hline 29 & Argon Blanket (Spare) & & & \\
\hline 30 & Temperature Comp. For R1-LT-530 & R1-TC-530 & EB-1-29020-F & \\
\hline 31 & $\begin{array}{l}\text { Argon Blanket (In T/C Well) No } \\
\text { Readout }\end{array}$ & R1-TC-505 & EB-1-29015-E & \\
\hline 32 & Bulk Sodium (Level dP Type) & R1-LT-530 & EB-1-25584-D & \\
\hline 33 & Bulk Sodium (Level Probe Type) & R1-LT-531 & EB-1-25582-D & \\
\hline 34 & Bulk Sodium (Level Float Type) & R1-LT-530R & & \\
\hline
\end{tabular}




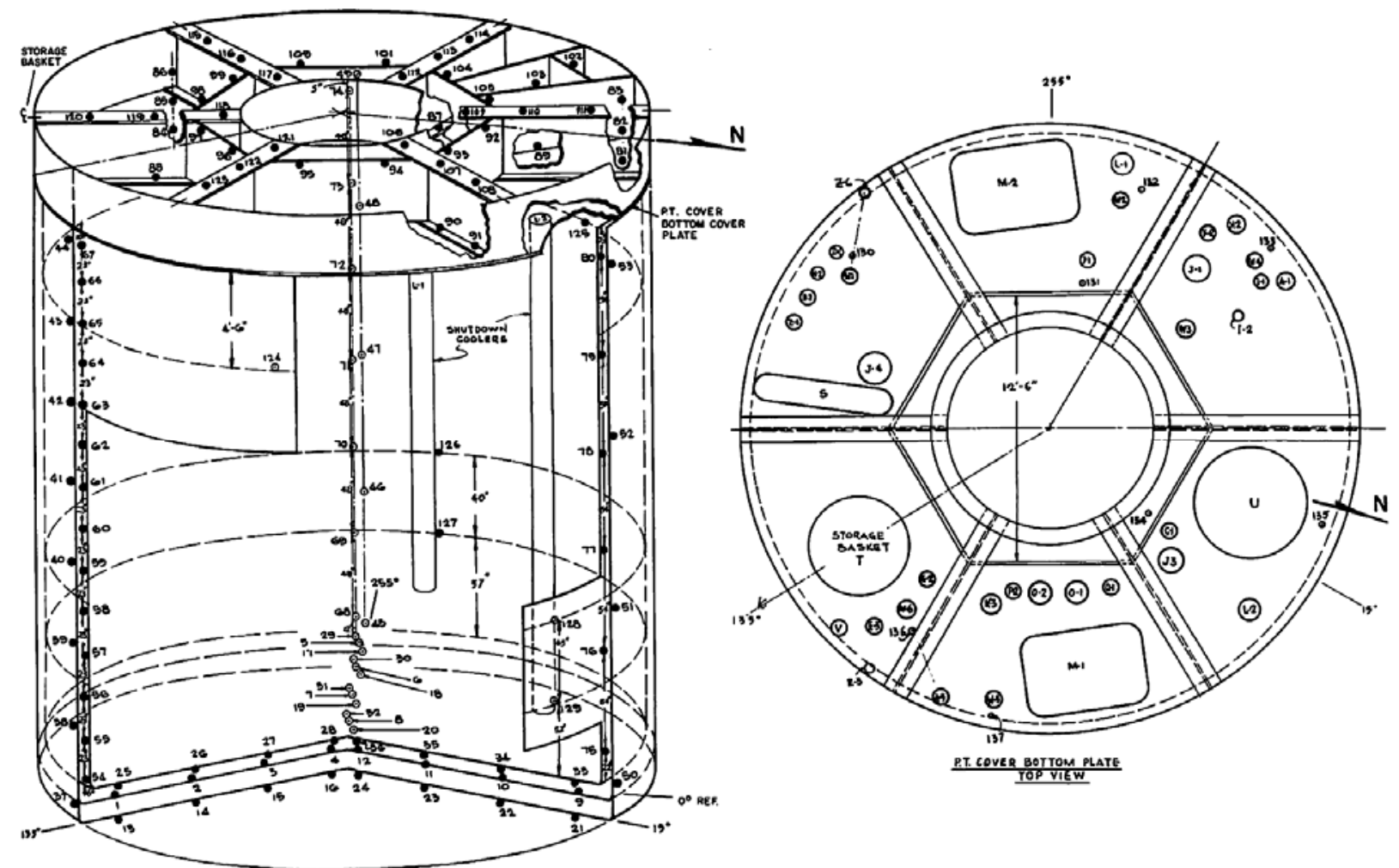

Figure 13 Figure 6.3-10 Thermocouples on Primary Tank [Vol. II, Chapter 6, pp.6.3-29/30] 
Table 17 Figure 6.3-10 Thermocouples on Primary Tank [Vol. II, Chapter 6, pp.6.3-29/30] (1/4)

\begin{tabular}{|c|c|c|c|c|c|}
\hline $\begin{array}{l}\text { Ref. } \\
\text { No. }\end{array}$ & Location & Instrument No. & & Ref. Dwg. & $\begin{array}{l}\text { Readout Inst. } \\
\text { Point Numbers }\end{array}$ \\
\hline 1 & Outer Vessel Inside Bottom & R1-TC-507A & $\square$ & EB-1-25272-D & 1 \\
\hline 2 & & R1-TC-507B & $\square$ & & 2 \\
\hline 3 & & R1-TC-507C & $\square$ & & 3 \\
\hline 4 & & R1-TC-507D & $\square$ & & 4 \\
\hline 5 & & R1-TC-507E & $\square$ & & 27 \\
\hline 6 & & R1-TC-507F & $\square$ & & 28 \\
\hline 7 & & R1-TC-507DV & $\square$ & & 5 \\
\hline 8 & & R1-TC-507DW & $\square$ & & 6 \\
\hline 9 & & R1-TC-507DX & $\square$ & & 29 \\
\hline 10 & & R1-TC-507DY & $\square$ & & 30 \\
\hline 11 & & R1-TC-507DZ & $\square$ & & 31 \\
\hline 12 & $\downarrow$ & R1-TC-507EA & $\square$ & & 32 \\
\hline 13 & Outer Vessel Outside Bottom & R1-TC-540FR & $\Delta$ & & 72 \\
\hline 14 & & R1-TC-540FS & $\Delta$ & & 73 \\
\hline 15 & & R1-TC-540FT & $\Delta$ & & 74 \\
\hline 16 & & R1-TC-540FV & $\Delta$ & & 75 \\
\hline 17 & & R1-TC-540FW & $\Delta$ & & 76 \\
\hline 18 & & R1-TC-540FX & $\Delta$ & & 77 \\
\hline 19 & & R1-TC-540FY & $\Delta$ & & 78 \\
\hline 20 & & R1-TC-540FZ & $\Delta$ & & 79 \\
\hline 21 & & R1-TC-540GA & $\Delta$ & & 80 \\
\hline 22 & & R1-TC-540GB & $\Delta$ & & 81 \\
\hline 23 & & R1-TC-540GC & $\Delta$ & & 82 \\
\hline 24 & $\downarrow$ & R1-TC-540GD & $\Delta$ & $\downarrow$ & 83 \\
\hline 25 & Inner Vessel Outside Bottom & R1-TC-507BB & $\square$ & EB-1-25260-D & 44 \\
\hline 26 & & R1-TC-507BC & $\square$ & & 45 \\
\hline 27 & & R1-TC-507BD & $\square$ & & 46 \\
\hline 28 & & R1-TC-507BE & $\square$ & & 47 \\
\hline 29 & & R1-TC-507AP & $\square$ & & 33 \\
\hline 30 & & R1-TC-507AQ & $\square$ & & 34 \\
\hline 31 & & R1-TC-507AR & $\square$ & & 35 \\
\hline 32 & & R1-TC-507AS & $\square$ & & 36 \\
\hline 33 & & R1-TC-507AB & $\square$ & & 23 \\
\hline 34 & & R1-TC-507AC & $\square$ & & 24 \\
\hline 35 & & R1-TC-507AD & $\square$ & & 25 \\
\hline 36 & $\downarrow$ & R1-TC-507AE & $\square$ & $\nabla$ & 26 \\
\hline 37 & Outer Vessel Shell Outside & R1-TC-540GE & $\Delta$ & EB-1-25272-D & 84 \\
\hline 38 & Wall & R1-TC-540GF & $\Delta$ & & 85 \\
\hline 39 & & R1-TC-540GG & $\Delta$ & & 86 \\
\hline 40 & & R1-TC-540GH & $\Delta$ & & 87 \\
\hline 41 & $\downarrow$ & R1-TC-540GK & $\Delta$ & $\downarrow$ & 88 \\
\hline
\end{tabular}


Table 17. Figure 6.3-10 Thermocouples on Primary Tank [Vol. II, Chapter 6, pp.6.3-29/30] (2/4)

\begin{tabular}{|c|c|c|c|c|c|}
\hline $\begin{array}{l}\text { Ref. } \\
\text { No. }\end{array}$ & Location & Instrument No. & & Ref. Dwg. & $\begin{array}{c}\text { Readout Inst. } \\
\text { Point Numbers }\end{array}$ \\
\hline 42 & $\begin{array}{c}\text { Outer Vessel Shell Outside } \\
\text { Wall }\end{array}$ & R1-TC-540GM & $\bar{\Delta}$ & EB-1-25272-D & 89 \\
\hline 43 & & R1-TC-540GN & $\Delta$ & & 90 \\
\hline 44 & & R1-TC-540GP & $\Delta$ & & 91 \\
\hline 45 & & R1-TC-540GQ & $\Delta$ & & 92 \\
\hline 46 & & R1-TC-540GR & $\Delta$ & & 93 \\
\hline 47 & & R1-TC-540GS & $\Delta$ & & 94 \\
\hline 48 & & R1-TC-540GT & $\Delta$ & & 95 \\
\hline 49 & & R1-TC-540GV & $\Delta$ & & 96 \\
\hline 50 & & R1-TC-540GW & $\Delta$ & & 97 \\
\hline 51 & & R1-TC-540GX & $\Delta$ & & 98 \\
\hline 52 & & R1-TC-540GY & $\Delta$ & & 99 \\
\hline 53 & $\downarrow$ & R1-TC-540GZ & $\Delta$ & $\downarrow$ & 100 \\
\hline 54 & Inner Vessel Shell Outside & R1-TC-507BF & $\square$ & EB-1-25266-D & 48 \\
\hline 55 & Wall & R1-TC-507BG & $\square$ & & 49 \\
\hline 56 & & R1-TC-507BH & $\square$ & & 50 \\
\hline 57 & & R1-TC-507BK & $\square$ & & 51 \\
\hline 58 & & R1-TC-507G & $\square$ & & 7 \\
\hline 59 & & R1-TC-507H & $\square$ & & 8 \\
\hline 60 & & R1-TC-507K & $\square$ & & 9 \\
\hline 61 & & R1-TC-507M & $\square$ & & 10 \\
\hline 62 & & R1-TC-507N & $\square$ & & 11 \\
\hline 63 & & R1-TC-507P & $\square$ & & 12 \\
\hline 64 & & R1-TC-507Q & $\square$ & & 13 \\
\hline 65 & & R1-TC-507R & $\square$ & & 14 \\
\hline 66 & & R1-TC-507S & $\square$ & & 15 \\
\hline 67 & & R1-TC-507T & $\square$ & & 16 \\
\hline 68 & & R1-TC-507AT & $\square$ & & 37 \\
\hline 69 & & R1-TC-507AV & $\square$ & & 38 \\
\hline 70 & & R1-TC-507AW & $\square$ & & 39 \\
\hline 71 & & R1-TC-507AX & $\square$ & & 40 \\
\hline 72 & & R1-TC-507AY & $\square$ & & 41 \\
\hline 73 & & R1-TC-507AZ & $\square$ & & 42 \\
\hline 74 & & R1-TC-507BA & $\square$ & & 43 \\
\hline 75 & & R1-TC-507V & $\square$ & & 17 \\
\hline 76 & & R1-TC-507W & $\square$ & & 18 \\
\hline 77 & & R1-TC-507X & $\square$ & & 19 \\
\hline 78 & & R1-TC-507Y & $\square$ & & 20 \\
\hline 79 & & R1-TC-507Z & $\square$ & & 21 \\
\hline 80 & $\nabla$ & R1-TC-507AA & $\square$ & $\downarrow$ & 22 \\
\hline 81 & Cover SHLDG Balls & R1-TC-540AW & $\triangle$ & EB-1-25179-F & 39 \\
\hline 82 & $\downarrow$ & R1-TC-540AX & $\triangle$ & $\downarrow$ & 40 \\
\hline
\end{tabular}


Table 17. Figure 6.3-10 Thermocouples on Primary Tank [Vol. II, Chapter 6, pp.6.3-29/30] (3/4)

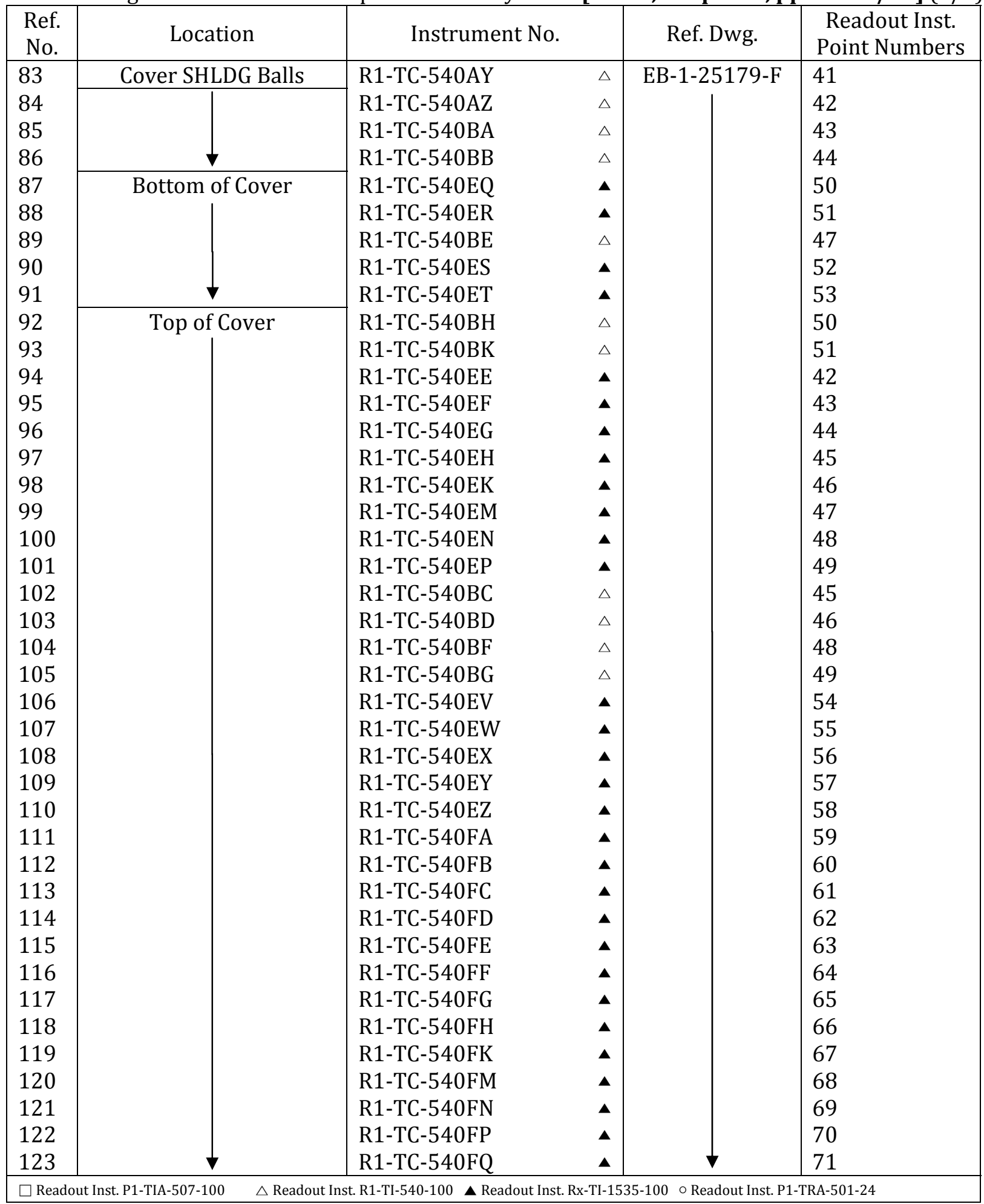


Table 17. Figure 6.3-10 Thermocouples on Primary Tank [Vol. II, Chapter 6, pp.6.3-29/30] (4/4)

\begin{tabular}{|c|c|c|c|c|}
\hline $\begin{array}{l}\text { Ref. } \\
\text { No. }\end{array}$ & Location & Instrument No. & Ref. Dwg. & $\begin{array}{l}\text { Readout Inst. } \\
\text { Point Numbers }\end{array}$ \\
\hline 124 & $\begin{array}{l}\text { P.T. Wall at Electric HTR. Plug } \\
\text { W-5 4'-6" Below P.T. Cover } \\
\text { Bottom Plate }\end{array}$ & R1-TC-501A & EB-1-29079-E & 1 \\
\hline 125 & $\begin{array}{l}\text { P.T. Wall at Electric HTR. Plug } \\
\text { W-4 4'-6" Below P.T. Cover } \\
\text { Bottom Plate }\end{array}$ & R1-TC-501R & EB-1-29079-E & 14 \\
\hline 126 & $\begin{array}{l}\text { P.T. Wall 97" Above Bottom } \\
\text { Plate of Bottom Structure to } \\
\text { Shutdown Cooler Plug L-1 }\end{array}$ & R1-TC-540T & EB-1-29020-F & 16 \\
\hline 127 & $\begin{array}{l}\text { P.T. Wall 57" Above Bottom } \\
\text { Plate of Bottom Structure, } \\
\text { close to Shutdown Cooler } \\
\text { Plug L-1 }\end{array}$ & R1-TC-540V & EB-1-29020-F & 17 \\
\hline 128 & $\begin{array}{l}\text { P.T. Wall 97" Above Bottom } \\
\text { Plate of Bottom Structure to } \\
\text { Shutdown Cooler Plug L-2 }\end{array}$ & R1-TC-540Z & EB-1-29020-F & 21 \\
\hline 129 & $\begin{array}{l}\text { P.T. Wall 52" Above Bottom } \\
\text { Plate of Bottom Structure, } \\
\text { close to Shutdown Cooler } \\
\text { Plug L-2 }\end{array}$ & R1-TC-540AA & EB-1-29020-F & 22 \\
\hline 130 & $\begin{array}{l}\text { P.T. Cover Bottom Plate 8" out } \\
\text { from Side of W-1 HTR. Plug } \\
\text { on Line between CTRS of W-1 } \\
* \text { Z-6 Nozzles }\end{array}$ & R1-TC-501G & EB-1-29079-E & 7 \\
\hline 131 & $\begin{array}{l}\text { P.T. Cover Bottom Plate 12" } \\
\text { from Center of P-1 Nozzle } \\
\text { toward Center of P.T. }\end{array}$ & R1-TC-540R & EB-1-29020-F & 14 \\
\hline 132 & $\begin{array}{l}\text { P.T. Cover Bottom Plate near } \\
\text { W-2 HTR. }\end{array}$ & R1-TC-540Q & EB-1-29020-F & 13 \\
\hline 133 & $\begin{array}{l}\text { P.T. Cover Bottom Plate } \\
\text { between W-4 HTR. and P.T. } \\
\text { Wall }\end{array}$ & R1-TC-501AB & EB-1-29079-E & 19 \\
\hline 134 & $\begin{array}{l}\text { P.T. Cover Bottom Plate at } \\
\text { Point 15" from Center of C-1 } \\
\text { Nozzle toward Center of P.T. }\end{array}$ & R1-TC-540X & EB-1-29020-F & 19 \\
\hline 135 & $\begin{array}{l}\text { P.T. Cover Bottom Plate at } \\
\text { Pointe between Heat } \\
\text { Exchanger and P.T. Wall }\end{array}$ & R1-TC-540W & EB-1-29020-F & 18 \\
\hline 136 & $\begin{array}{l}\text { P.T. Cover Bottom Plate at } \\
\text { Point 6" out from Side of W-6 } \\
\text { HTR. Plug toward A-5 Nozzle }\end{array}$ & R1-TC-501AC & EB-1-29079-E & 10 \\
\hline 137 & $\begin{array}{l}\text { P.T. Cover Bottom Plate at } \\
\text { Point between W-5 HTR. Plug } \\
\text { * P.T. Wall }\end{array}$ & R1-TC-540G & EB-1-29020-F & 7 \\
\hline
\end{tabular}




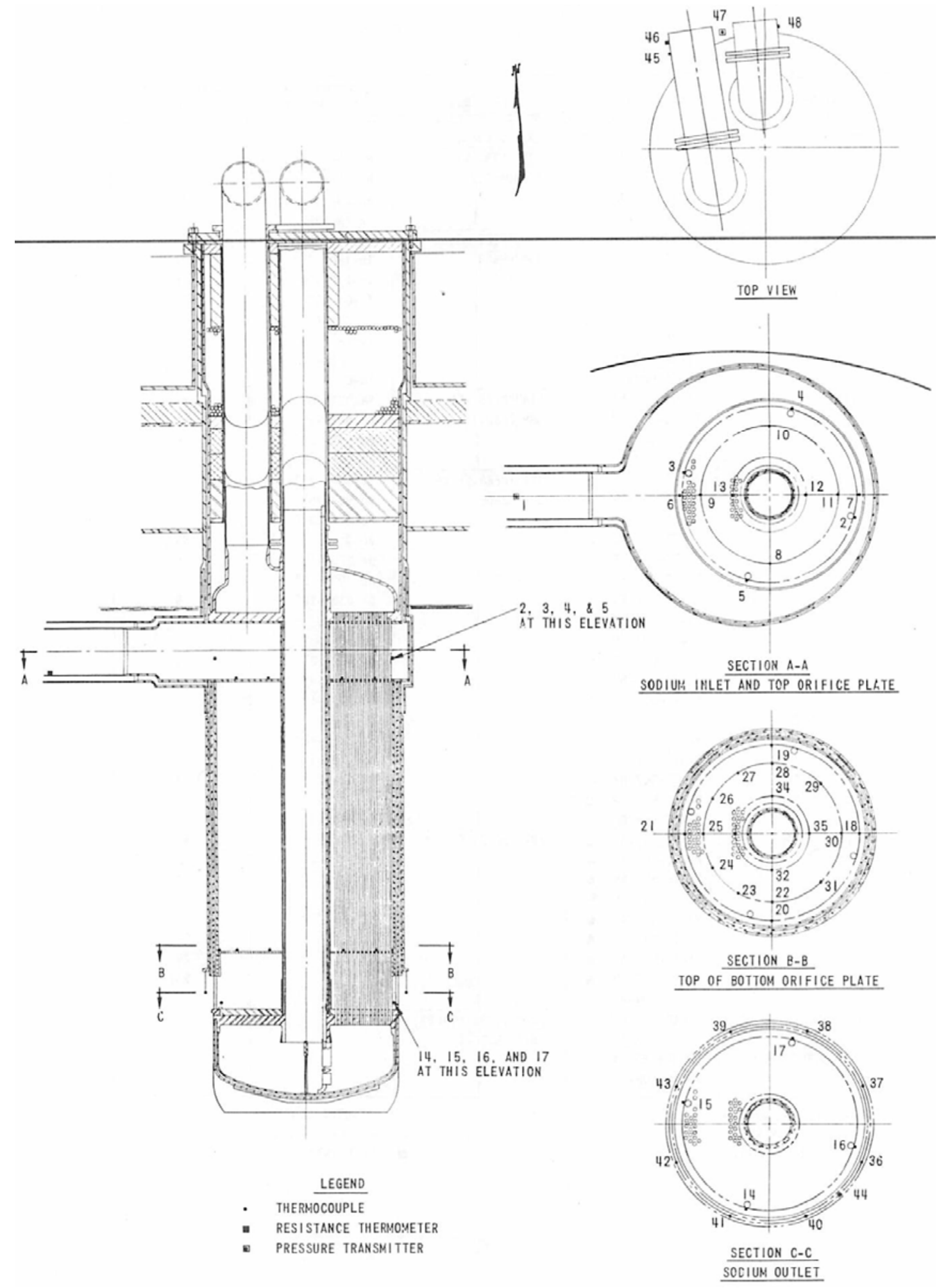

Figure 14 Figure 6.3-11 Intermediate Heat Exchanger [Vol. II, Chapter 6, pp.6.3-33/34] 
Table 18 Figure 6.3-11 Intermediate Heat Exchanger [Vol. II, Chapter 6, pp.6.3-33/34]

\begin{tabular}{|c|c|c|c|c|c|}
\hline $\begin{array}{l}\text { Ref. } \\
\text { No. }\end{array}$ & Location & Instrument No. & Ref. Dwg. & $\begin{array}{l}\text { Struthers Wells } \\
\text { T.C. MK. No. }\end{array}$ & $\begin{array}{l}\text { Readout Inst. } \\
\text { Point No. }\end{array}$ \\
\hline 1 & \multirow{5}{*}{ PRI. Na Inlet } & R1-PT-522-K & EB-1-29016-E & \multirow{5}{*}{$\begin{array}{l}\text { Na IN-195" } \\
\text { Na IN-15" } \\
\text { Na IN-105" } \\
\text { Na IN-285" }\end{array}$} & 9 \\
\hline 2 & & R1-TC-507-BQ & Struthers-Wells & & 55 \\
\hline 3 & & R1-TC-507-BKA $\square$ & 59P8088D17 & & 9 \\
\hline 4 & & R1-TC-507-BN & & & 53 \\
\hline 5 & & R1-TC-507-BS & $\nabla$ & & 57 \\
\hline 6 & \multirow{8}{*}{ Top Orifice Plate } & R1-TC-507-CP & Struthers-Wells & To- $0^{\circ}-25^{\prime \prime}$ & 75 \\
\hline 7 & & R1-TC-507-CQ & 59P8088D4 & Тo-180-25” & 76 \\
\hline 8 & & R1-TC-507-EQ & & Тo-270-19” & 8 \\
\hline 9 & & R1-TC-507-CM & & Тo- $0^{\circ}-19^{\prime \prime}$ & 73 \\
\hline 10 & & R1-TC-507-EP & & То- $90^{\circ}-19^{\prime \prime}$ & 7 \\
\hline 11 & & R1-TC-507-CN & & Тo-180-19” & 74 \\
\hline 12 & & R1-TC-507-CK & & Тo- $180^{\circ}-9^{\prime \prime}$ & 72 \\
\hline 13 & & R1-TC-507-CH & $\nabla$ & To- $0^{\circ}-9 "$ & 71 \\
\hline 14 & \multirow{4}{*}{ PRI. Na Outlet } & R1-TC-507-BT & Struthers-Wells & Na Out- $285^{\circ}$ & 58 \\
\hline 15 & & R1-TC-507-BM & 59P8088D17 & Na Out $-15^{\circ}$ & 52 \\
\hline 16 & & R1-TC-507-BR & & Na Out- $195^{\circ}$ & 56 \\
\hline 17 & & R1-TC-507-BP & $\downarrow$ & Na Out $-105^{\circ}$ & 54 \\
\hline 18 & \multirow{18}{*}{ Bottom Orifice Plate } & R1-TC-507-CF & Struthers-Wells & Bo $180^{\circ}-25^{\prime \prime}$ & 69 \\
\hline 19 & & R1-TC-507-CA & 59P8088D3 & Во $90^{\circ}-25^{\prime \prime}$ & 64 \\
\hline 20 & & R1-TC-507-CG & & Bo $270^{\circ}-25^{\prime \prime}$ & 70 \\
\hline 21 & & R1-TC-507-BZ & & Bo $0^{\circ}-25^{\prime \prime}$ & 63 \\
\hline 22 & & R1-TC-507-CE & & Bo $270^{\circ}-19^{\prime \prime}$ & 68 \\
\hline 23 & & R1-TC-507-EM & & Bo $300^{\circ}-19^{\prime \prime}$ & 5 \\
\hline 24 & & R1-TC-507-EN & & Bo $330^{\circ}-19^{\prime \prime}$ & 6 \\
\hline 25 & & R1-TC-507-BX & & Bo $0^{\circ}-19^{\prime \prime}$ & 61 \\
\hline 26 & & R1-TC-507-EF & & Вo $30^{\circ}-19^{\prime \prime}$ & 1 \\
\hline 27 & & R1-TC-507-EG & & Bo $60^{\circ}-19^{\prime \prime}$ & 2 \\
\hline 28 & & R1-TC-507-BY & & Во $90^{\circ}-19^{\prime \prime}$ & 62 \\
\hline 29 & & R1-TC-507-EH & & Bo $135^{\circ}-19^{\prime \prime}$ & 3 \\
\hline 30 & & R1-TC-507-CD & & Bo $180^{\circ}-19^{\prime \prime}$ & 67 \\
\hline 31 & & R1-TC-507-EK & & Bo $225^{\circ}-19^{\prime \prime}$ & 4 \\
\hline 32 & & R1-TC-507-CC & & Bo $270^{\circ}-9^{\prime \prime}$ & 66 \\
\hline 33 & & R1-TC-507-BV & & Bo $0^{\circ}-9^{\prime \prime}$ & 59 \\
\hline 34 & & R1-TC-507-BW & & Bo $90^{\circ}-9 "$ & 60 \\
\hline 35 & & R1-TC-507-CB & $\nabla$ & Bo $180^{\circ}-9^{\prime \prime}$ & 65 \\
\hline 36 & \multirow{9}{*}{ PRI. Na Outlet } & R1-TC-540-AQ & EB-1-29020-F & & 34 \\
\hline 37 & & R1-TC-540-AP & & & 33 \\
\hline 38 & & R1-TC-540-AN & & & 32 \\
\hline 39 & & R1-TC-540-AM & & & 31 \\
\hline 40 & & R1-TC-540-AE & & & 26 \\
\hline 41 & & R1-TC-540-AD & & & 25 \\
\hline 42 & & R1-TC-540-AC & & & 24 \\
\hline 43 & & R1-TC-540-AB & & & 23 \\
\hline 44 & & R1-RT-504-A & $\nabla$ & & 1 \\
\hline 45 & \multirow{2}{*}{ SEC. Na Outlet Pipe } & R2-TC-508-F & R2-RT-533B(Inlet) & & \\
\hline 46 & & R2-RT-533-A & EB-1-25250-C & & \\
\hline 47 & SEC. Na Inlet-Outlet Pipes & R2-dPT-525-A & & & 1 \\
\hline 48 & SEC. Na Inlet Pipe & R2-TC-508-G C & $\downarrow$ & & \\
\hline $\begin{array}{l}\Delta \mathrm{Re} \\
\Delta \mathrm{R} \\
\mathrm{OP} 1\end{array}$ & $\begin{array}{l}\text { out Inst. P1-TIA-507-100 } \\
\text { lout Inst. RX-TI-1535-100 } \\
\text { TRA-504 } \square \text { P2-PRA525- }\end{array}$ & $\begin{array}{l}\text { A Readout Inst. R1-' } \\
\text { Readout Inst. DAS } \\
2 \text { \& S2-PRA525-12 }\end{array}$ & P1-PRA522-12 & & \\
\hline
\end{tabular}




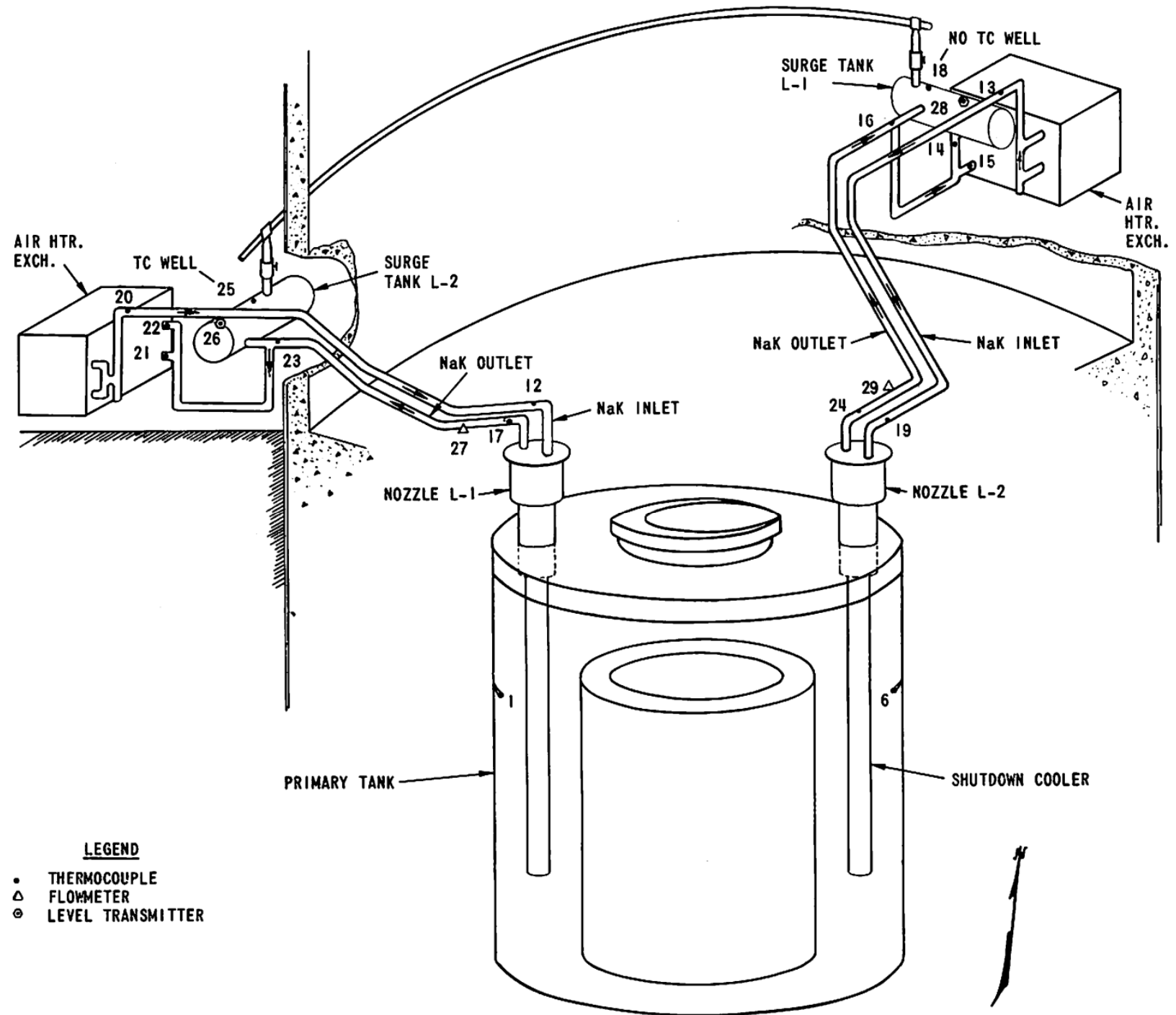

Figure 15 Figure 6.3-18 Shutdown Coolers [Vol. II, Chapter 6, pp.6.3-43/44] 
Table 19 Figure 6.3-18 Shutdown Coolers [Vol. II, Chapter 6, pp.6.3-43/44]

\begin{tabular}{|c|c|c|c|c|c|}
\hline $\begin{array}{l}\text { Ref. } \\
\text { No. }\end{array}$ & Location & Instrument No. & Reference Drawing & $\begin{array}{l}\text { Inst. } \\
\text { No. }\end{array}$ & $\begin{array}{c}\text { Point. } \\
\text { No }\end{array}$ \\
\hline 1 & Near L1- Shutdown Cooler & R1-TC-540-S & EB-1-29020-F & 540 & 15 \\
\hline 6 & Near L-2 Shutdown Cooler & R1-TC-540-Y & EB-1-29020-F & 540 & 20 \\
\hline 12 & NaK Inlet to L-1 Shutdown Cooler & R4-TC-511-AG & HKF-R803, R806 & 511 & 3,2 \\
\hline 13 & NaK Outlet from L-2 Air HTR Exch. & R4-TC-511-BD & & & 3,15 \\
\hline 14 & NaK Inlet to L-2 Air HTR Exch. & R4-TC-511-AR & & $\downarrow$ & 3,10 \\
\hline 15 & NaK Inlet to L-2 Air HTR Exch. & R4-TC-511-AS & & & \\
\hline 16 & NaK Inlet to L-2 Air HTR Exch. & R4-TC-511-AV & & & \\
\hline 17 & NaK Outlet from L-1 Shutdown Cooler & R4-TC-511-AK & & 511 & 3,5 \\
\hline 18 & Loose in Cooler \#2 & R4-TC-511-AN & & & 3,7 \\
\hline 19 & NaK Inlet to L-2 Shutdown Cooler & R4-TC-511-AF & & & 3,1 \\
\hline 20 & NaK Outlet from L-1 Air HTR Exch. & R4-TC-511-BC & & $\nabla$ & 3,14 \\
\hline 21 & NaK Inlet to L-1 Air HTR Exch. & R4-TC-511-AQ & & & \\
\hline 22 & NaK Inlet to L-1 Air HTR Exch. & R4-TC-511-AP & & 511 & 3,8 \\
\hline 23 & NaK Inlet to L-1 Air HTR Exch. & R4-TC-511-AT & & & \\
\hline 24 & NaK Outlet from L-2 Shutdown cooler & R4-TC-511-AH & & 511 & 3,4 \\
\hline 25 & Loose in Cooler \#1 & R4-TC-511-AM & & $\downarrow$ & 3,6 \\
\hline 26 & On Surge Tank L-1 Air HTR Exch. & R4-LT-529-A & & $529 \mathrm{~A}$ & \\
\hline 27 & NaK Outlet from L-1 Shutdown Cooler & R4-FM-518-A & & $518 \mathrm{~A}, 5$ & \\
\hline 28 & Surge Tank Level L-2 Air HTR Exch. & R4-LT-529-B & & $529 B$ & \\
\hline 29 & NaK Outlet from L-2 Shutdown Cooler & R4-FM-518-B & $\nabla$ & 518B, 5 & \\
\hline
\end{tabular}

\section{Drawings of Sensors in EBR-II System}

To measure the reactor conditions, five types of instruments are employed. Thermocouples and resistance thermometers were used to measure the temperatures in the reactor system. The flow rate of the reactor coolant was measured by the magnetic flowmeters. Volumetric pressure sensing elements and differential pressure sensing elements were used to detect the system pressures. Fission-counter and ionchamber were installed in the thimble to detector the neutron flux. The physical drawings of each instrument are shown in following sections.

\subsection{Thermocouples and Thermometers}

Figure 16 and Figure 17 show the pipe surface thermocouple assembly and pipe surface thermocouple mounting, respectively.

Figure 18 shows thermocouple assembly for the thermowell. Figure 19 and Figure 20 show the schematic of typical thermowell and typical thermowell type thermocouple mounting, respectively.

Figure 21 shows the special thermocouple assembly. Figure 22 shows the "type A" and the "type B" of special thermocouple, respectively.

Resistance thermometers were used to measure the temperatures of Sodium coolant and air. The thermometers for the Sodium and the air are shown in Figure 23 and Figure 24, respectively. 


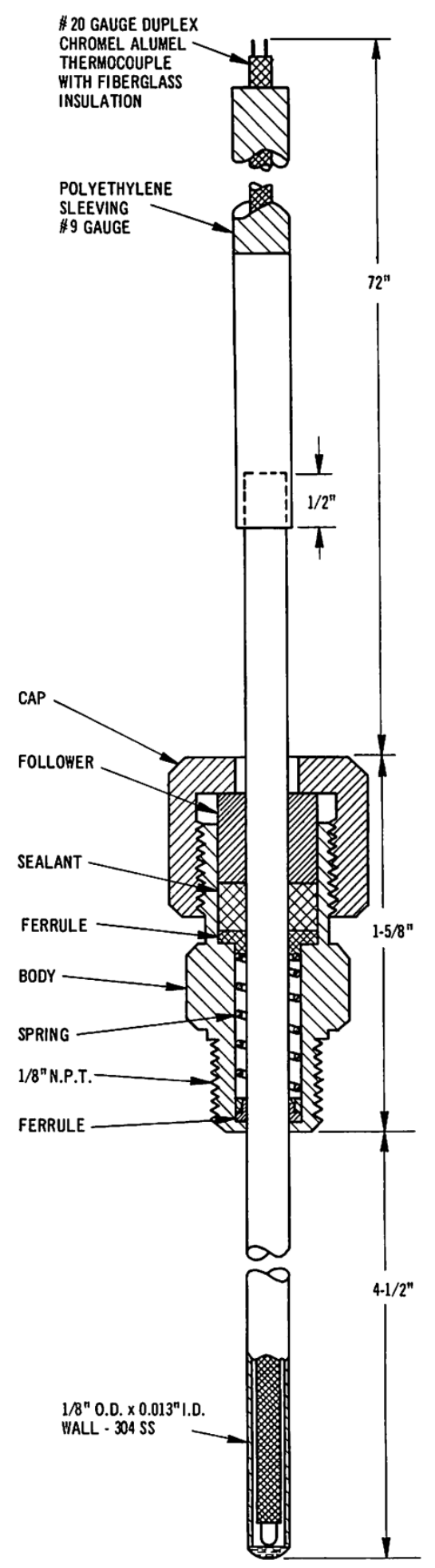

Figure 16 Figure 6.9-2 Pipe Surface Thermocouple Assembly [Vol. II, Chapter 6, p.6.9-5] 


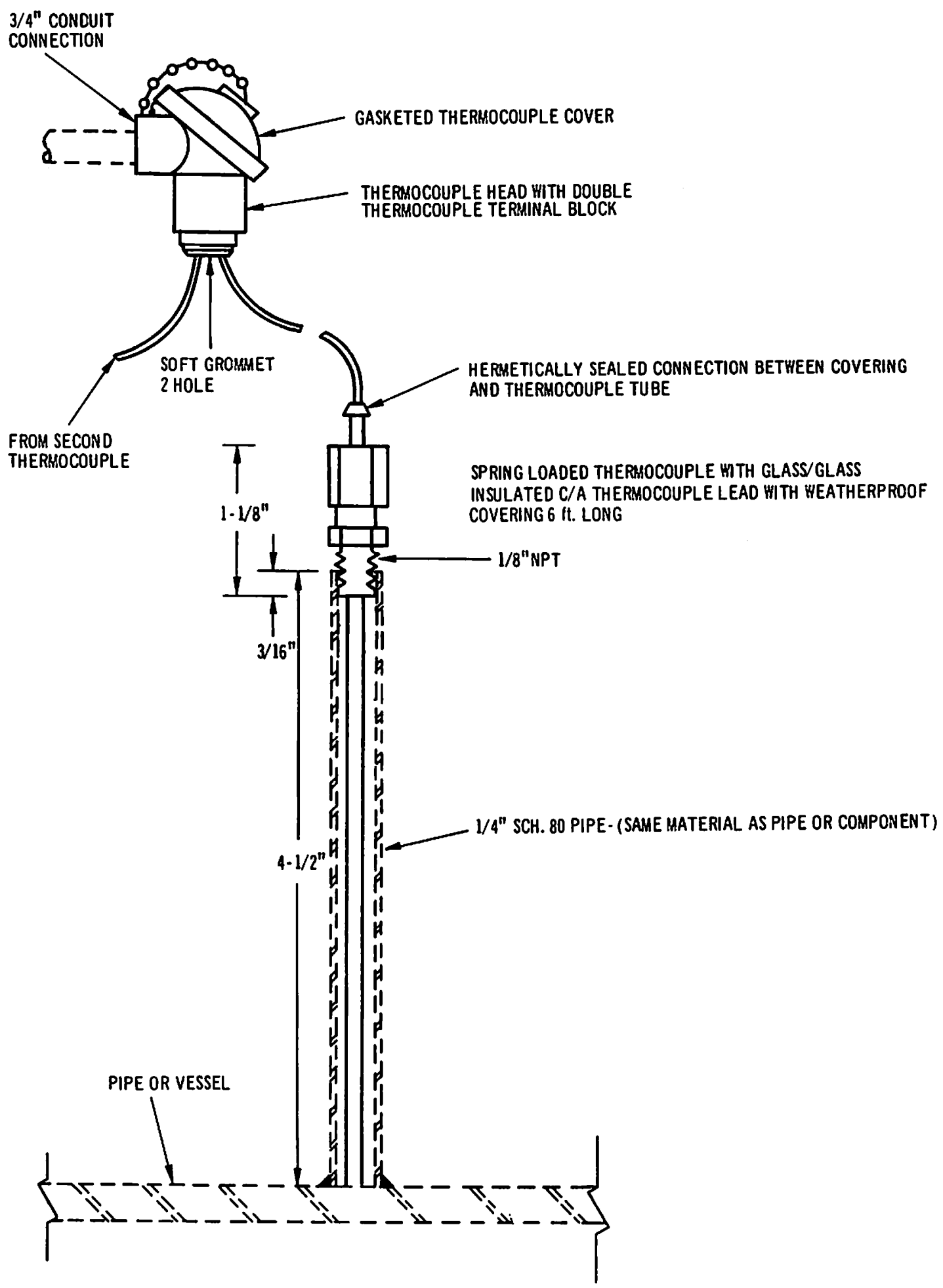

Figure 17 Figure 6.9-3 Pipe Surface Thermocouple Mounting [Vol. II, Chapter 6, p.6.9-6] 


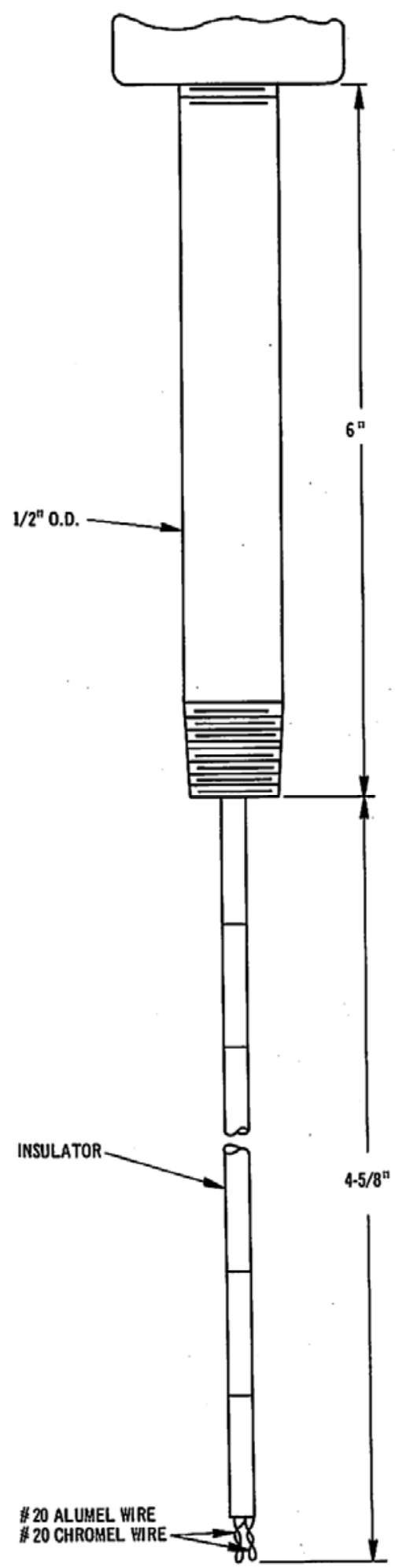

Figure 18 Figure 6.9-4 Thermocouple Assembly for Thermowell [Vol. II, Chapter 6, p.6.9-7] 


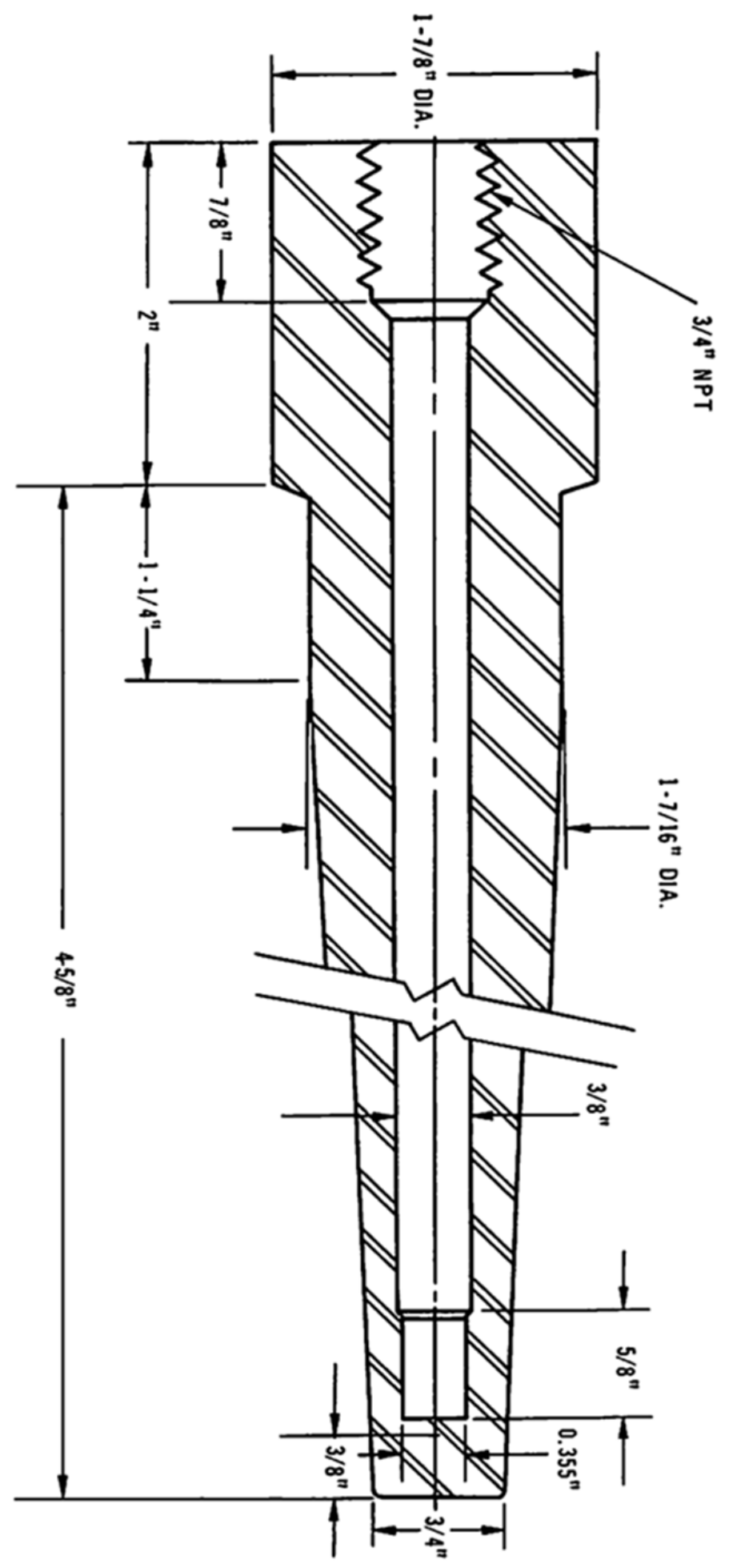

Figure 19 Figure 6.9-5 Typical Thermowell [Vol. II, Chapter 6, p.6.9-9] 


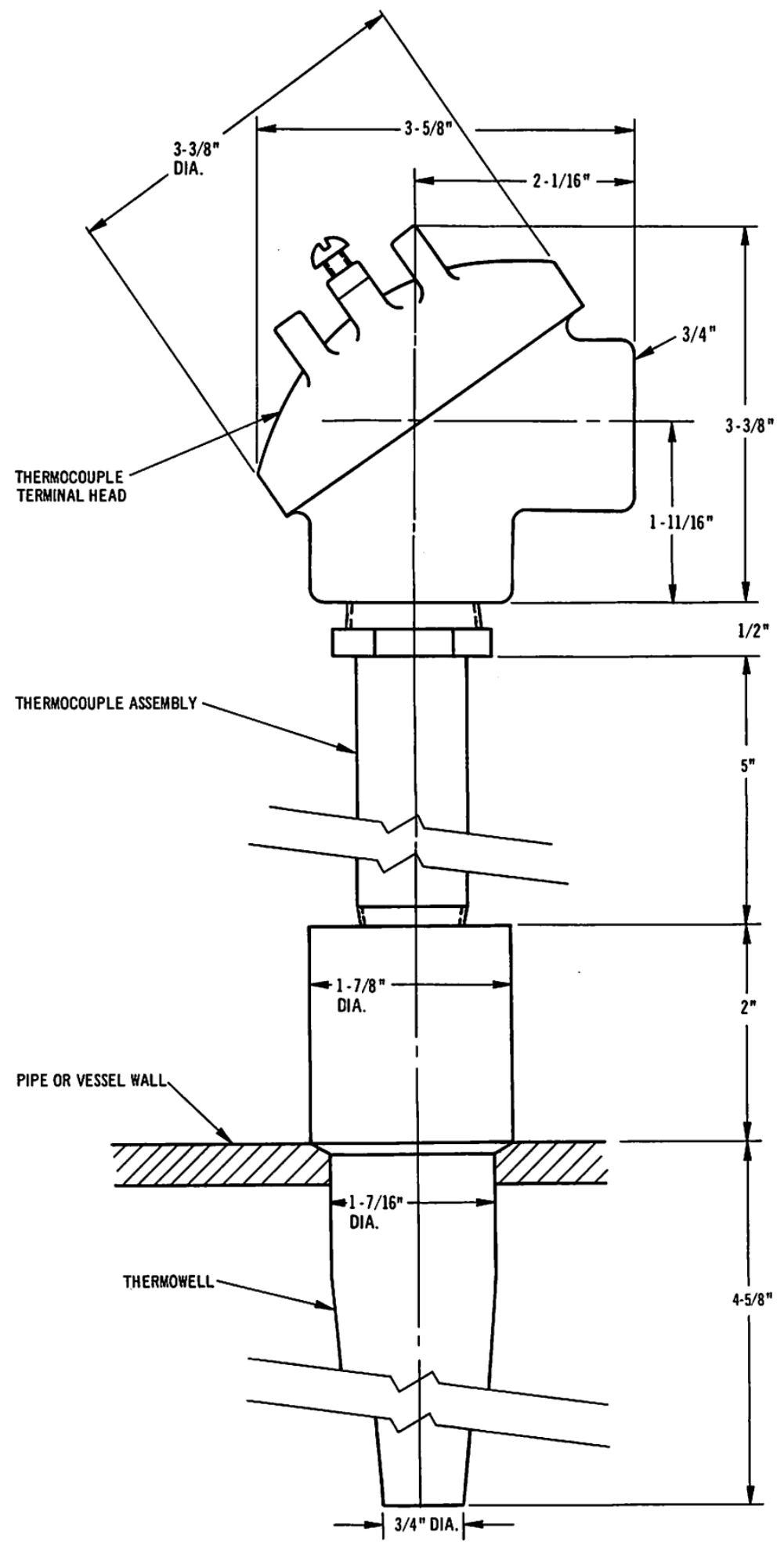

Figure 20 Figure 6.9-6 Typical Thermowell Type Thermocouple Mounting [Vol. II, Chapter 6, p.6.9-10] 


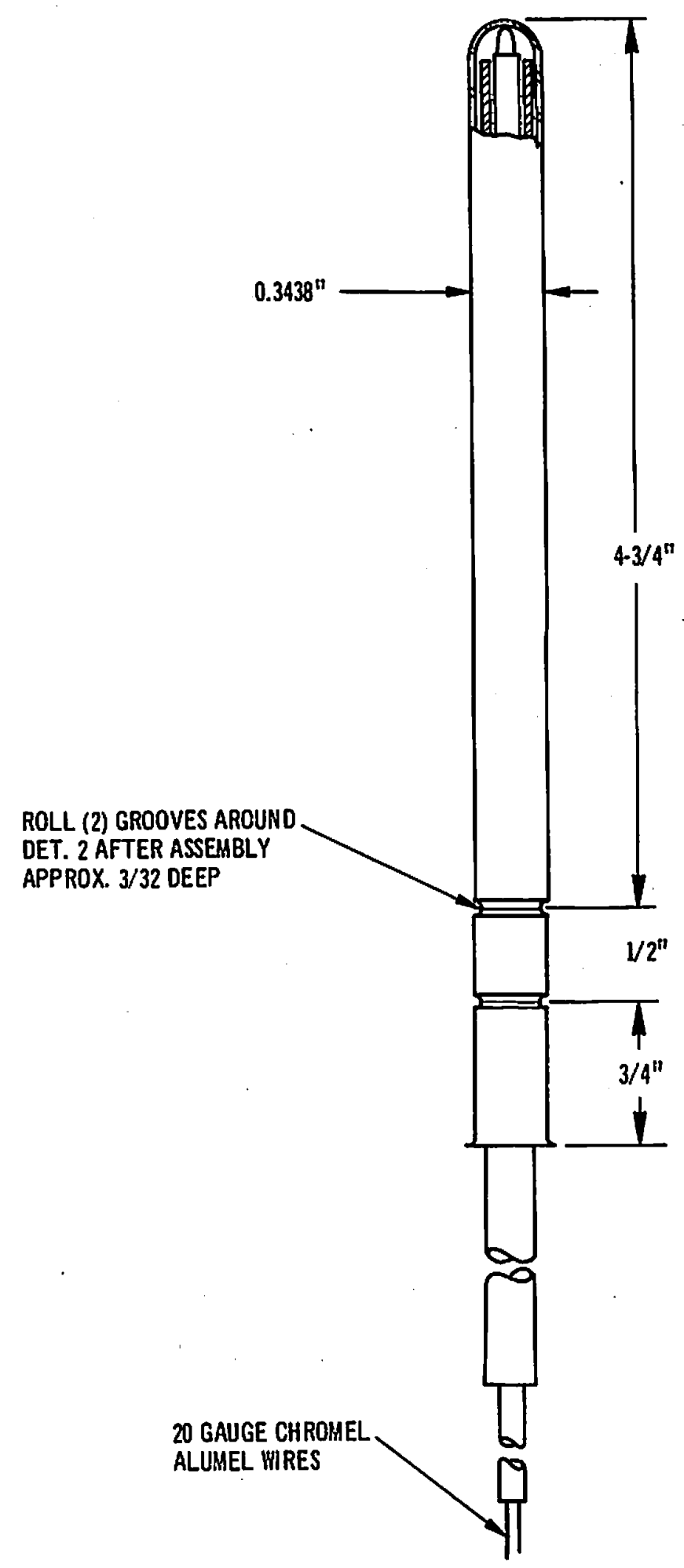

Figure 21 Figure 6.9-7 Special Thermocouple Assembly [Vol. II, Chapter 6, p.6.9-11] 


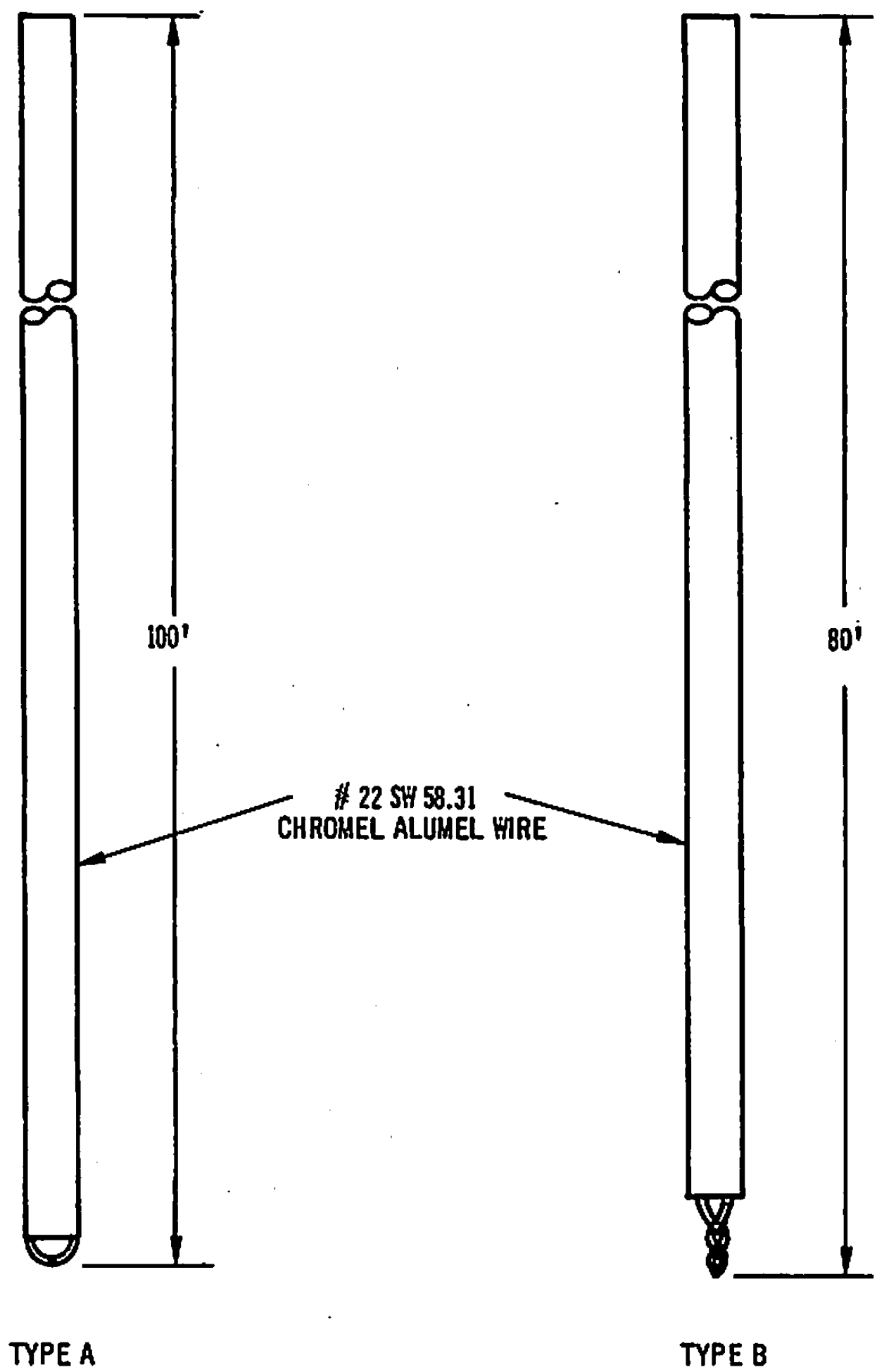

Figure 22 Figure 6.9-8 Special Thermocouple [Vol. II, Chapter 6, p.6.9-13] 


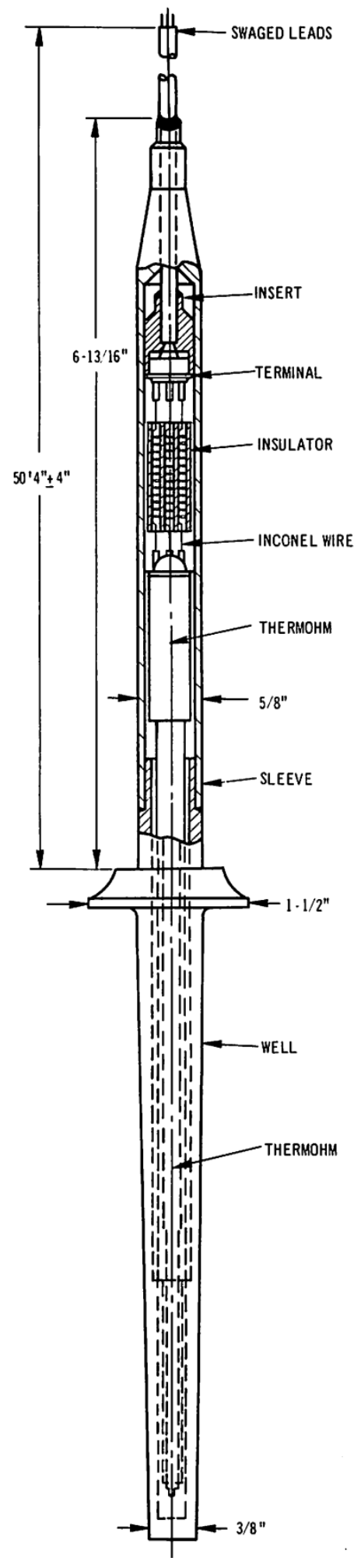

Figure 23 Figure 6.9-35 Typical Resistance Thermometer for High Temperature Use in Sodium [Vol. II, Chapter 6, p.6.9-103] 


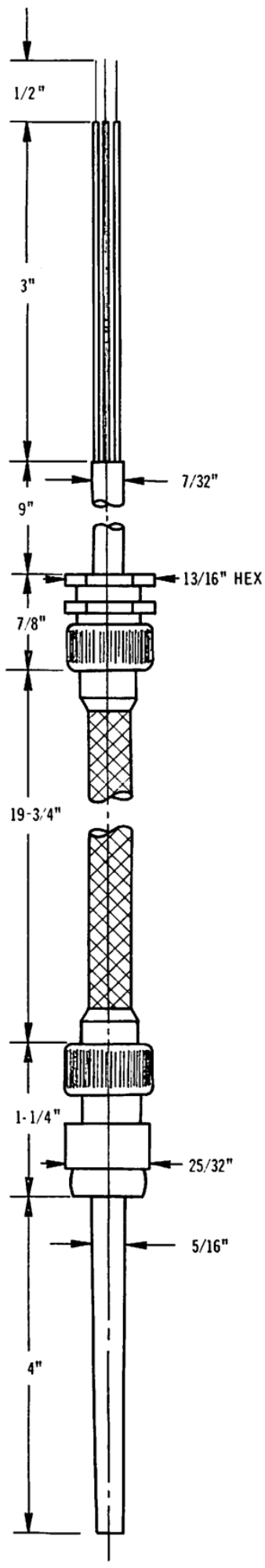

Figure 24 Figure 6.9-36 Resistance Thermometer for Sensing Air Temperatures [Vol. II, Chapter 6, p.6.9-103] 


\subsection{Pressure Sensors}

NaK filled volumetric pressure sensing element manufactured by Taylor Instrument Co. was used to measure the local pressures in the reactor system. The differential pressure sensing head was used to detect the differential pressure in the primary system components. NaK filled volumetric pressure sensing elements and differential pressure sensing head are presented in Figure 25and Figure 26, respectively.

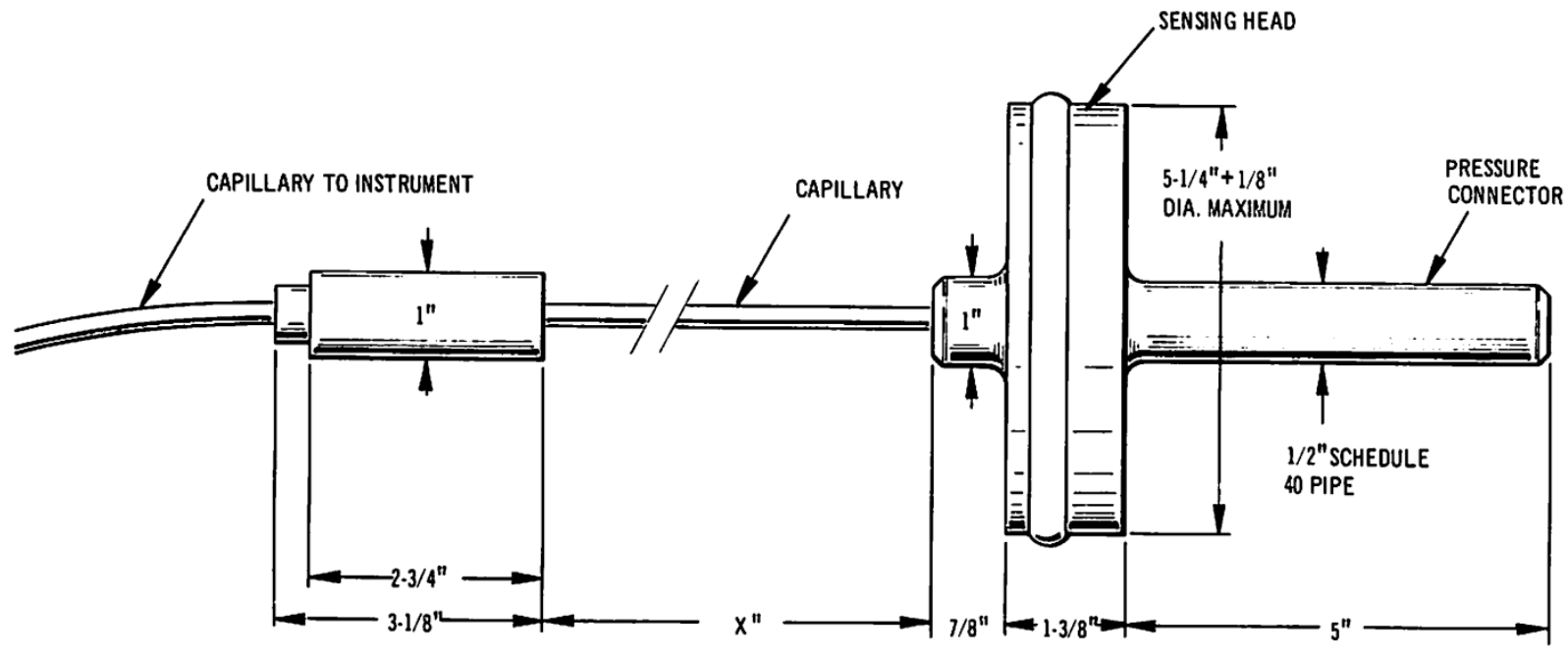

Figure 25 Figure 6.10-2 NaK Filled Volumetric Pressure Sensing Element [Vol. II, Chapter 6, p.6.10-5]

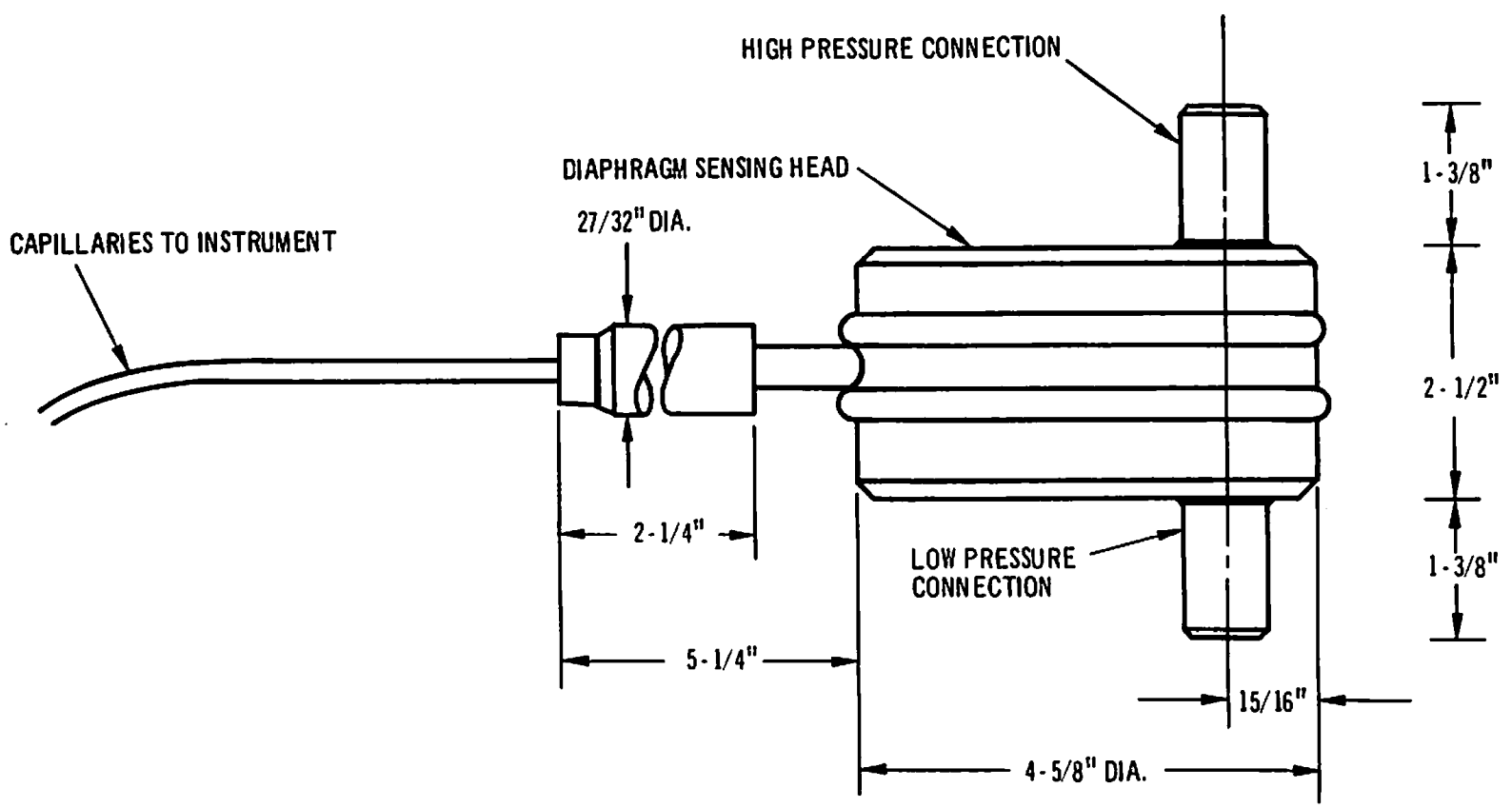

Figure 26 Figure 6.10-5 Differential Pressure Sensing Head [Vol. II, Chapter 6, p.6.10-13] 


\subsection{Flowmeters}

Magnetic flowmeter and flow tube are used to measure the flow rate of Sodium coolant in primary system. Figure 27 shows the magnetic flowmeter installation for large sodium cooling pipe. Small magnetic flowmeter was installed in the sodium purification system as shown in Figure 28. Figure 29 shows the magnetic flowmeter installed below the capsule bundle to measure Sodium flow through the subassembly.

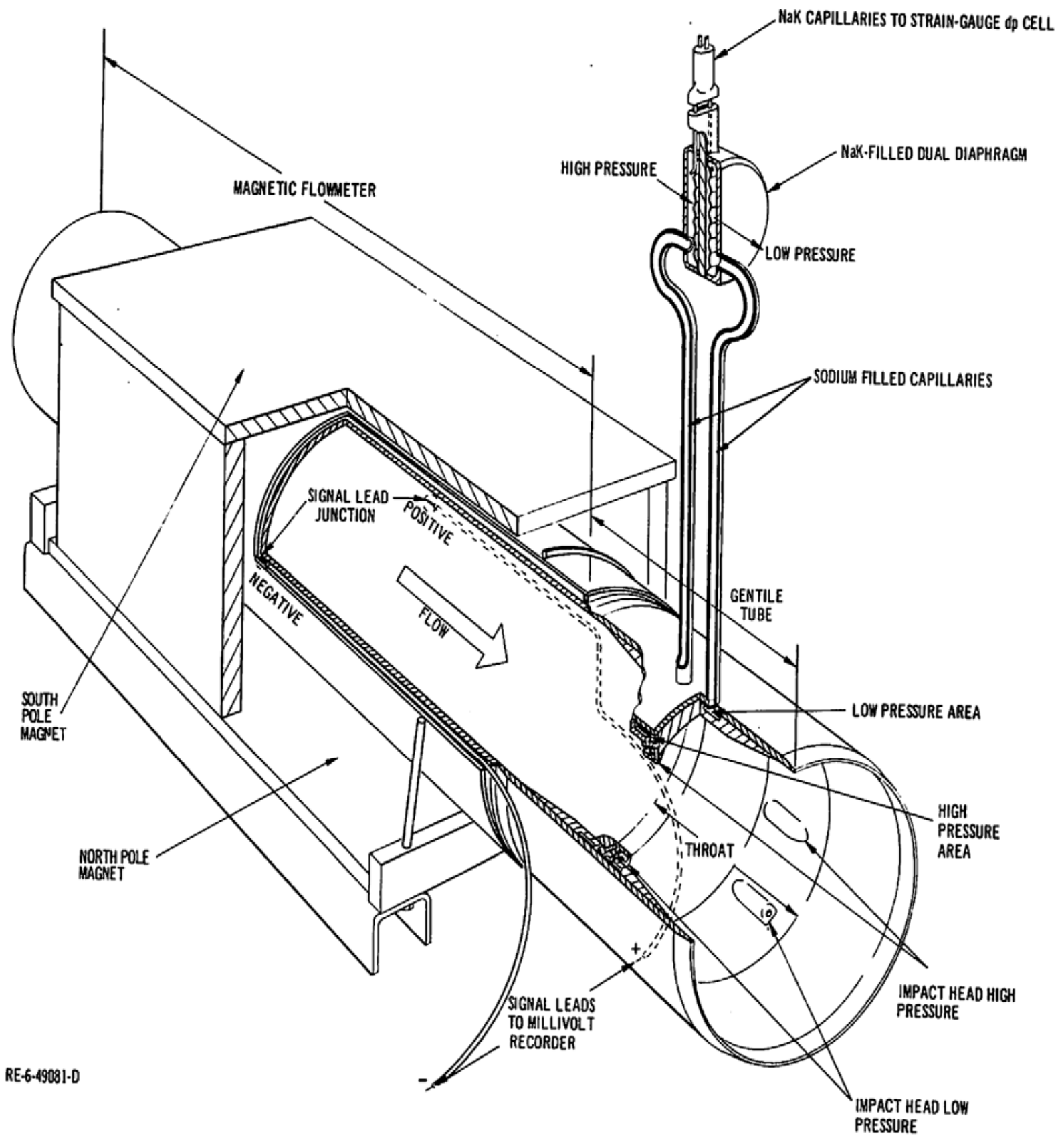

Figure 27 Figure 6.11-1 Magnetic Flowmeter and Flow Tube for Large Sodium Coolant Piping [Vol. II, Chapter 6, p.6.11-3] 


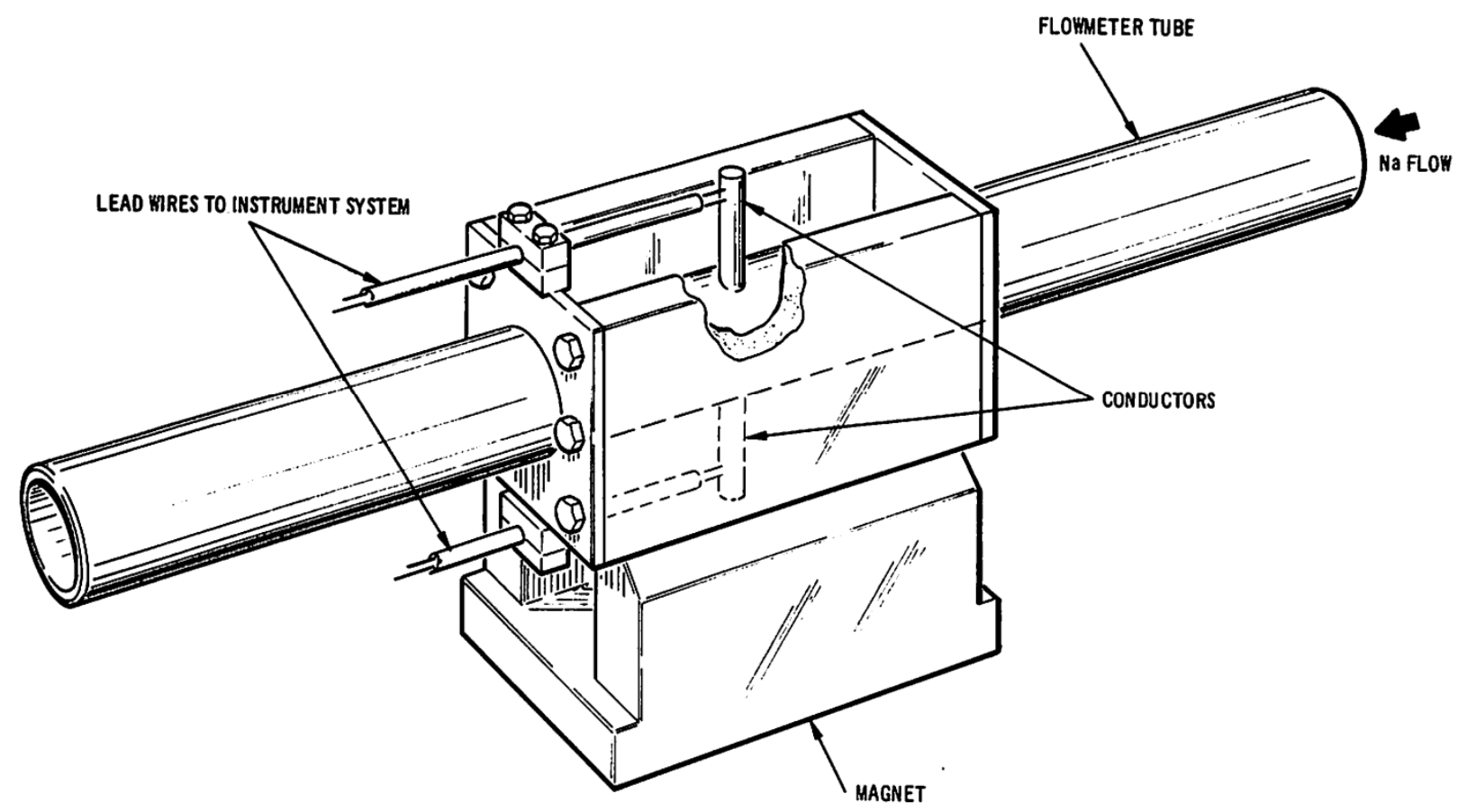

Figure 28 Figure 6.11-8 Small Magnetic Flowmeter Installation - Sodium Purification Flowmeters, R1FM-515-A and R1-FM-515-B [Vol. II, Chapter 6, p.6.11-17] 


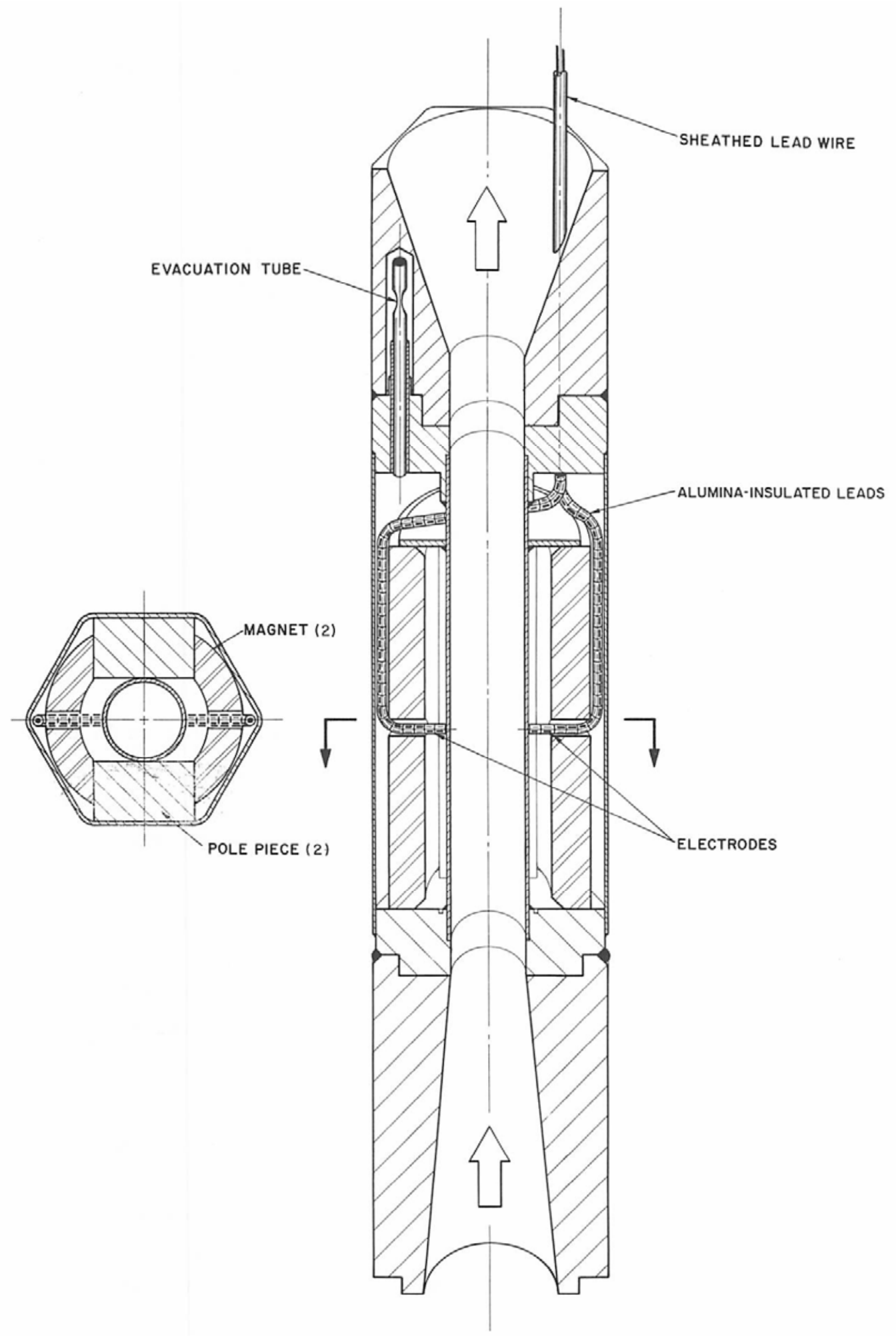

Figure 29 Figure 6.15-27 Magnetic Flowmeter [Vol. II, Chapter 6, p.6.15-77] 


\subsection{Level Sensors}

Figure 30 shows the installation of resistance probes to measure the sodium level in the primary tank. Two kinds of level sensing elements were employed to measure the sodium level in primary tank: a differential pressure type level transmitter and a float type level transmitter. Figure 30 and Figure 31 show the differential pressure type and float type level transmitters, respectively.

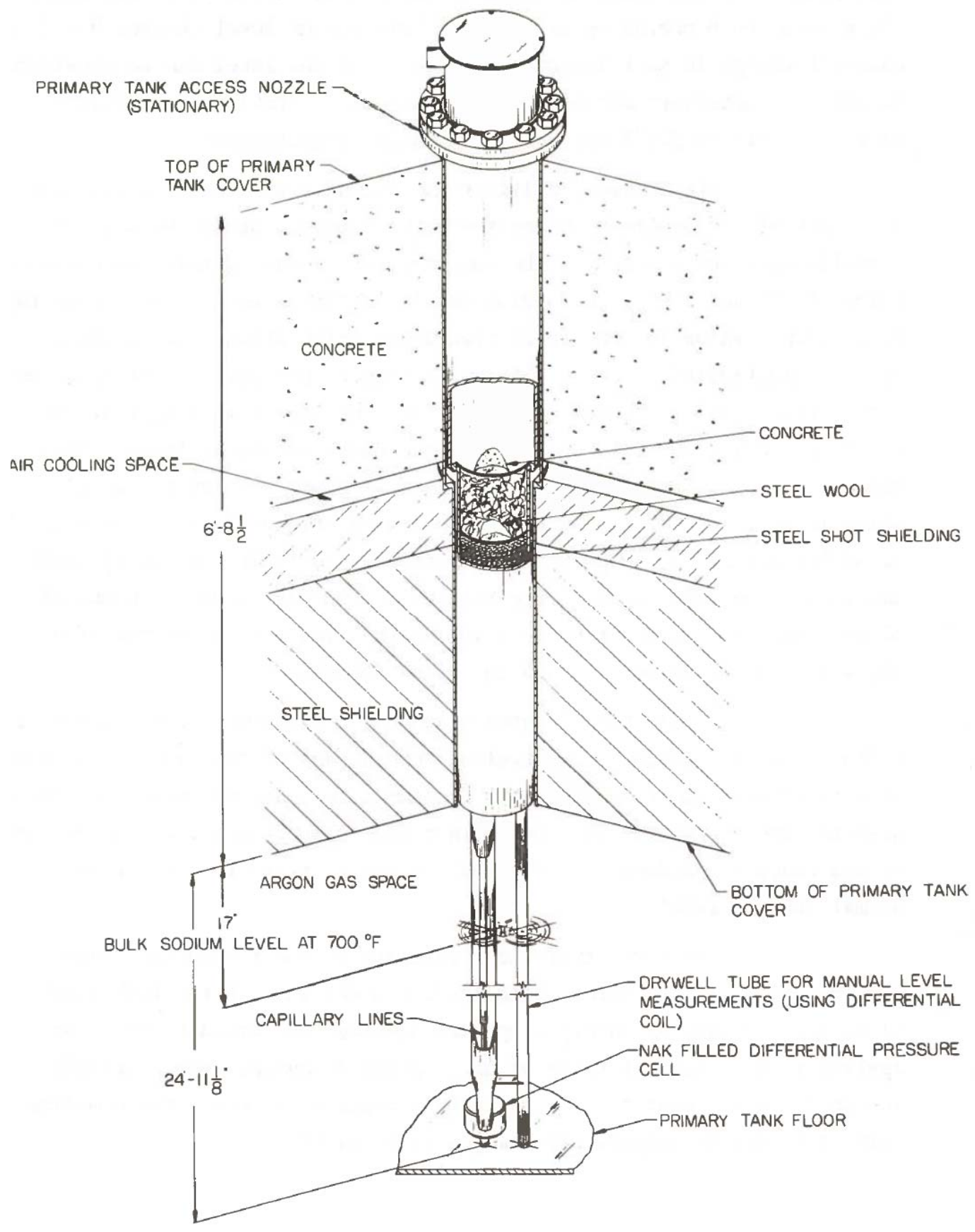

Figure 30 Figure 6.12-3 Differential Pressure Type Level Transmitter [Vol. II, Chapter 6, p.6.12-7] 


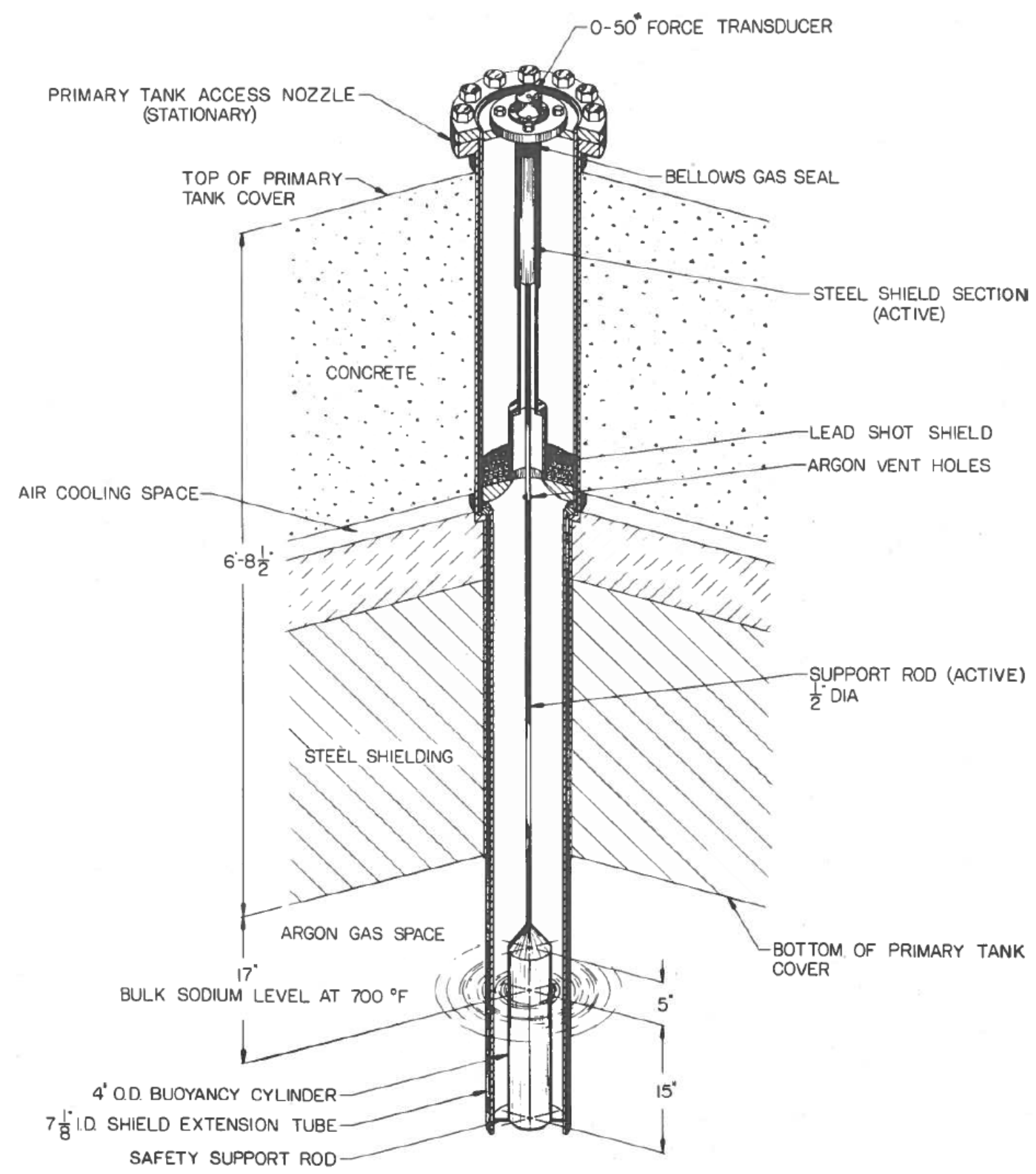

Figure 31 Figure 6.12-4 Float Type Level Transmitter [Vol. II, Chapter 6, p.6.12-9] 


\subsection{Flux-monitor Tube}

Figure 32 shows the installation of flux-monitor tube. This sealed tube is build to allow the insertion of a small diameter flux monitor where the self-powered neutron detector (Reuter Stokes Model RSN202-MI) is inserted.

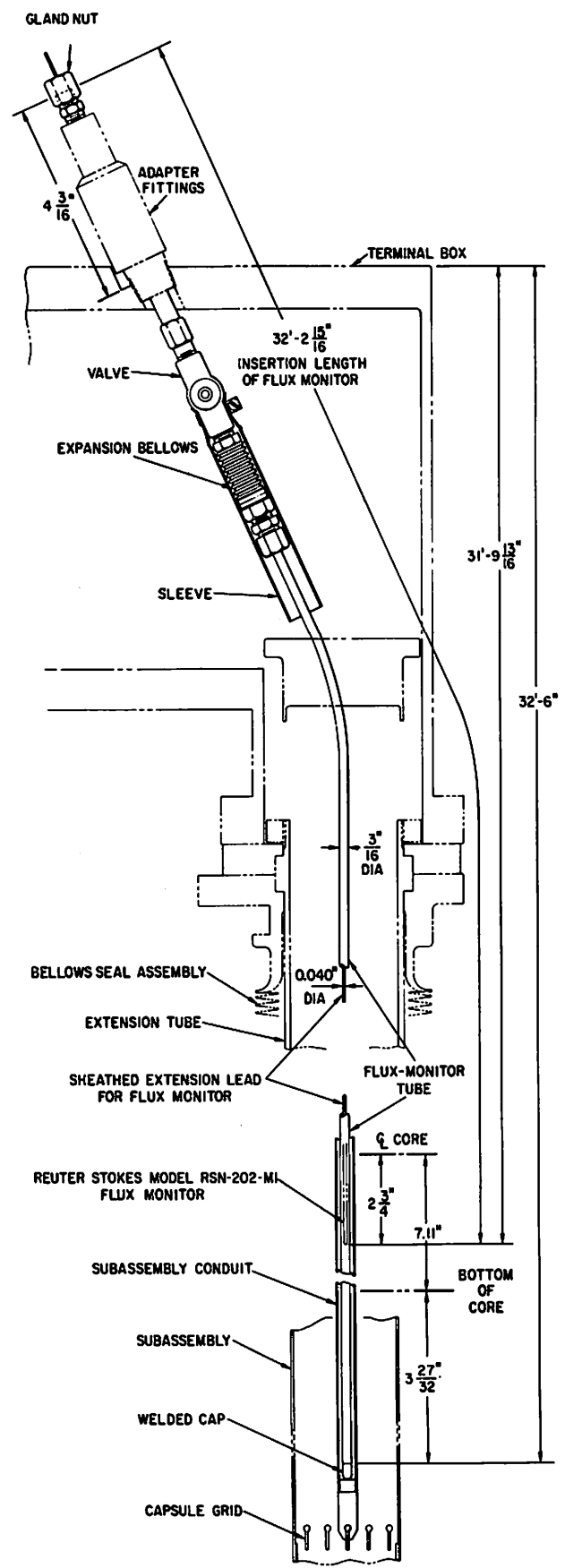

Figure 32 Figure 2.11-11 Installation of Flux-monitor Tube [Vol. II, Chapter 2, p.2.11-28] 


\section{Descriptions of the Sensors}

In Chapter 2, Chapter3 and Chapter 6 of Volume II, it is described the detailed information of the sensors in the primary system. In this section, the descriptions for each sensor are summarized chapter by chapter.

\subsection{Volume II, Chapter 2}

\subsubsection{Thermocouple}

Volume II, Pages: 2.11-23) Thermocouples are available to measure fuel-centerline, coolant, and structural-material temperatures. Two-types of thermocouples are used: chromel/alumel thermocouples to measure coolant and structural-material temperatures, and tungsten- $3 \%$ rhenium/tungsten- $25 \%$ rhenium thermocouples to measure fuel-centerline temperatures.

The tungsten/rhenium fuel-centerline thermocouples have tantalum sheaths and thoria insulation. Reactor experience with this type of thermocouple is limited; however, this thermocouple provides useful temperature data below $2400^{\circ} \mathrm{F}$. The components of the tungsten/rhenium thermocouples are manufactured by Engelhard Industries Incorporated. These materials are guaranteed to $\pm 1 \%$ accuracy at the anticipated fuel-centerline temperatures. Fuel enrichment was selected to produce temperatures in the $1700 \sim 2000^{\circ} \mathrm{F}$ range.

The chromel/alumel coolant and structural thermocouples have Type 304 stainless steel sheaths and alumina insulation. The chromel and alumel materials are a special close-tolerance grade of Chromel-P and Alumel developed for precision applicants. These materials are guaranteed to register voltage values accurate to within $\pm 3 / 8 \%$ from $530^{\circ}$ to $2300^{\circ} \mathrm{F}$. This material is "Specification $3 \mathrm{G}-178$," manufactured by Hoskins Manufacturing Company. The chromel/alumel thermocouples have either 0.062-in. or 0.040-in. diameters. Thermocouples used to sense coolant inlet and outlet temperature are 0.062 in. in diameter. The 0.040-in.-ida thermocouples replace the normal spacer wires on some capsules. The spacer-wire thermocouples measure capsule-cladding temperature.

Volume II, Pages: 2.12-20) Two types of thermocouples are included in the FPTF: the regular type, and the fast-response type. The fast-response thermocouples have a time constant of $25 \mathrm{~ms}$ or less. The regular thermocouples have a response time of $250 \mathrm{~ms}$. The signals from all thermocouples are read out on the DAS. The fast-response thermocouples have a grounded junction, so their operability cannot be checked by checking insulation resistances. These thermocouples are connected to differential-input amplifier in the TIR. The primary output from the amplifiers is routed to the DAS expansion room patch panel, and the auxiliary output from the amplifiers is connected to the DAS in the TIR. Figure 2.12-7 shows a block diagram of the fast-response-thermocouple system. The regular thermocouples and the cable for the fast-response thermocouples were procured according to RDT Standard C7-6T; the junction of the fast-response thermocouple is not covered by this standard.

\subsubsection{Fission-gas Pressure Transducer}

Volume II, Pages 2.11-25) The fission-gas pressure transducer is a pressure-balancing type device used to measure buildup of fission-gas pressure in fueled capsules. This instrument contains two bellows joined by a coupling disk and an electrical contact probe. The inside of the lower bellows is directly connected to the fuel-element gas space. The inside of the upper bellows is connected to a reference-gas supply. Reference-gas pressure is read directly from a gauge and from a millivolt recorder via a straingauge transducer. The transducer controls the reference-gas pressure to the point where it equals the fission-gas pressure. Figure 2.11-9 is a block diagram of the pressure transducer; a typical capsule with a pressure transducer was shown in Figure 2.11-8. Reference-gas pressure is controlled by a solenoid valve. 
This valve admits the gas (argon) from a high-pressure source (a separate bottle supply regulated to 100 psig) into the pressure transducer. The solenoid valve (Hoke, model 590A320C, with a 1/32-in. body orifice) is controlled by the resistance between the transducer electrical probe and the bellows coupling disk. The transducer has a resolution of $\pm 1 / 4 \mathrm{psig}$ and an overall accuracy of \pm 8 psig over the full 0-350psig range of the instrument.

\subsubsection{Flowmeter}

Volume II, Pages 2.11-27) The flowmeter is a sealed unit that fits into the subassembly hexagonal can between the lower adapter and the capsule bundle. The magnetic field is provided by two Alnico 5 magnets operating in parallel with a total flux of 740 gauss at room temperature. The electrical leads for the flowmeter are routed through alumina-insulated, stainless-steel-sheathed cable. This cable passes through the capsule bundle via a conduit tube that replaces one capsule. The sheathed cable is welded into the top of the flowmeter. The sheathed leads are connected to Type 304 stainless steel conductors that are welded to the flowmeter electrodes. The Type 304 stainless steel wire and electrodes are used to minimize thermoelectric and thermal-expansion effects that could be encountered if dissimilar metals were used. The flowmeter has a sensitivity of $0.325 \mathrm{mV} / \mathrm{gpm}$ at $800^{\circ} \mathrm{F}$, or about $10.5 \mathrm{mV}$ output at a flow rate of 32 $\mathrm{gpm}$. The unit is flow-tested in sodium before installation in the subassembly.

Volume II, Pages 2.12-17: The total sodium flow from the subassembly through the FPTF is measured by eddy-current flowmeters. Two flowmeters are provided to ensure continued monitoring of subassembly flow if one flowmeter fails. The flowmeters and flowmeter electronics for the FPTF are essentially identical to the flowmeters and electronics used for the BFTF (Breached Fuel Test Facility). The flowmeters and electronic package were obtained from Kaman Science Corp. The flowmeter were calibrated in an existing sodium loop at ANL-East. Some modification of the loop and a special test section were required before the calibration runs were made. This test loop provided a wider range of flow and a greater degree of calibration accuracy than the test loop used for BFTF calibration. A weigh tank was used to calibrate the flowmeter.

\subsubsection{Flux-monitor Tube}

Volume II, Pages 2.11-27) A sealed tube is provided to allow the insertion of a small diameter flux monitor (see Figure 2.11-11). This tube extends from the terminal box, through the extension tube, and into the subassembly, where it terminates at the capsule support gird. The tube has a 3/16-in. OD and a 0.016-in. wall thickness. The bottom of the tube is sealed by a plug, and the top is connected to a ball valve in the terminal box. The valve extends through the terminal-box wall and is capped. It is operated by a sealed stem that passes through the terminal-box wall. The flux-monitor tube is filled with argon. A subminiature, 0.063-in.-OD, sodium-wire, self-powered neutron detector (Reuter Stokes Model RSN202-MI) is inserted in the tube. The flux-monitor leads are sealed to the top of the monitor-tube valve by a packing gland that replaces the cap.

\subsection{Volume II, Chapter 3}

\subsubsection{Flowmeter}

Volume II, Pages 3.2-2) Three methods of flow measurement are used in the EBR-II primary system. The first method uses Gentile tubes, manufactured by the Hammel Dahl/Foster Engineering Division of General Controls Company; the second method uses permanent-magnet flowmeters, manufactured by the Mine Safety Appliance Company. The third method uses microprocessor-controlled ultrasonic flowmeters built by the EBR-II and Electronics Division of ANL. Additional information on Flow 
Measurement System may be found in Volume II, Chapter 6, (Instrumentation). The Gentile tubes are a shortened variation of a venturi flow tube, with fluid impact heads positioned around the inside diameter of the venturi. Half of the impact heads face upstream; the order half face downstream. The differential pressure between the two sets of impact heads is used to measure fluid flow rate. The fluid flow is measured by applying the pressure from the impact heads to a NaK-filled differential-pressure transducer.

Volume II, Pages 3.2-4) The operation of magnetic flowmeters is based on the fact that an electrical current is produced when an electrical conductor is moved within a magnetic field. The EBR-II flowmeters use the liquid sodium itself as a conductor; the magnetic field is provided by a permanent magnet that is positioned around the pipe containing the sodium. Flowing through the pipe, the sodium cuts the magnetic lines of force; as a result, a D.C voltage appears on the pipe walls at right to the magnetic poles. This voltage is directly proportional to the velocity of the sodium. A pair of electrodes is welded to the pipe walls at right angles to the magnetic field.

Volume II, Pages 3.2-5a: Ultrasonic-flowmeter operation is based on the fact that the velocity of an ultrasonic wave is related to the media in which it travels. Therefore, if two ultrasonic transducers are fixed in a flowing fluid, the apparent velocity of an ultrasonic pulse between them is the vector sum of the ultrasonic velocity relative to the fluid, and the fluid velocity relative to the transducers. Because the shape and size of the piping system is known, this information can easily be converted to system flow. These flowmeters have been used commercially in many forms.

\subsubsection{Pressure Transducer}

Volume II, Pages 3.2-1: NaK-filled pressure transducers are used to measure the various pressures in the primary system. This type of pressure sensing device consists of a stainless steel diaphragm unit that has one side of the diaphragm exposed to the pressure that is to be measured. The other side of the diaphragm has a capillary tube completely filled with $\mathrm{NaK}$ connected to it. The opposite end of the capillary tube is connected to a small bellows. The bellows has one end solidly anchored, with the movable end connected to an electrical strain-gauge transducer. As sodium pressure is applied to the sodium-side of the diaphragm unit, the diaphragm flexes slightly. As the diaphragm flexes, a small amount of $\mathrm{NaK}$ is forced through the capillary tube into the bellows. The bellows applies the force from the NaK flow to the electrical strain gauge, which changes resistance in proportion to the original sodium pressure. This electrical resistance is translated into a pressure reading on the Control-Panel instruments. Additional information on the Pressure Transducer Systems can be found in Volume II, Chapter 6, (Instrumentation).

\subsubsection{Level Indicator}

Volume II, Pages: 3.2-6) Sodium level in the primary tank is measured by two methods. The first method uses a NaK-filled differential-pressure indicator at the bottom of the tank. The high-pressure side of the indicator is open to the sodium the low-pressure side is piped to the blanket gas space above the sodium surface. Sodium pressure at the tank bottom is directly proportional to the depth of sodium above the differential pressure cell. This system has proven reliable, but has a major disadvantage; the system cannot be zeroed for recalibration after the primary tank has been filled. Additional information on Level Measurement System may be found in Volume II, Chapter 6, (Instrumentation).

Volume II, Pages: 3.2-6: The second method of level indication uses electrical probes of various lengths that extend through the primary tank cover into the tank. Each probe is a stainless steel tube with a heavy copper conductor inside. The conductor is insulated from the tube except at the tip, where the tube and conductor are connected (the copper conductor is completely enclosed and is not exposed to sodium). The probes are of different lengths with each probe connected to an individual Wheatstone bridge circuit. 
The bridge circuits are balanced while the probes are not in equals the sum of the resistances of the stainless tube and copper conductor. As the sodium level rises in the tank and strikes a probe the resistance of the stainless tube (which is considerably higher than the copper conductor) decreases and unbalances the bridge circuit that closes a relay and lights an indicator.

\subsection{Volume II, Chapter 6}

\subsubsection{Fission Counter}

Volume II, Pages: 6.4-1: The nuclear instrumentation system monitors the EBR-II reactor power by measuring the intensity of neutron flux during all modes of reactor operation including shutdown maintenance, fuel handling, reactor startup, approach to power, and full power operation. It converts the detected neutron flux into informational displays, and, is an emergency or unsafe flux condition, opens trip contacts in the plant protection system for the safe shutdown of the reactor.

Originally eleven nuclear instrument channels were provided to monitor the large range of neutron flux. Channels 1, 2 and 3, startup channels, monitored neutron source flux levels; channel 4, 5, and 6, monitored intermediate power range; channels 9, 10, and 11, power level channels, monitored high neutron flux levels; and channels 7 and $7 \mathrm{a}$, linear level channels, monitored neutron flux in approximately the same rages as the intermediate and high power level channels. Detectors for the eleven channels were housed in the eight air cooled thimbles located around the cylindrical portion of the reactor within the neutron shield at the approximate centerline of the reactor core.

Under Plant Modification No. 753, startup channels 1, 2 and 3, intermediate channel 4, 5, and 6, and power level channels 9,10 , and 11 were replaced with a wide-range nuclear instrumentation system consisting of three identical, redundant, and independent wide-range channels, each of which covers the 10 decades of neutron flux range of the EBR-II. Each wide range channel has three circuits that measure a different neutron flux range. The three measuring circuits are: log count rage (LCR) which covers a range of 1 count per second (cps) to $2 \times 10^{5} \mathrm{cps}$; average magnitude squared (AMS) which covers a range of $2 \times 10^{4} \mathrm{nv}$ to $2 \times 10^{10} \mathrm{nv}$; and linear power level (dc current range) which covers a range of 0 to $125 \%$ power. Also, the LCR and AMS signals are summed to provide a signal that covers 10 decades of neutron flux from $2 \mathrm{nv}$ to $2 \times 10^{10} \mathrm{nv}$.

Volume II, Pages: 6.4-2) Channels 7 and 7a have not been changed. These linear level channels cover a range of about $50 \mathrm{w}$ to $110 \mathrm{Mw}$. Figure 6.4-1 shows the range of all nuclear instrumentation circuits.

P.6.4-2: Detectors for the three wide-range channels and for channels 7 and 7 a are located in three "J" thimbles (J-1, J-3 and J-4). Design of the thimbles and thimble cooling is discussed in Volume II, Chapter 7.

Volume II, Pages: 6.4-2) The three wide-range channel guarded fission chamber detector assemblies, which have an overall length less than 20 in. and an O.D. of less than 4 in., are located in the "J" thimbles: Channel A in J-1, B in J-3, and C in J-4. The fission chambers are mounted at an elevation that provides $\sim 1 \times 1010 \mathrm{nv}$ of detected neutrons at $62.5 \mathrm{MW}(\mathrm{t})$. The detector output signal and the high voltage input are routed through separate shielded $35 \mathrm{ft}$. long coaxial cable in a $1 / 2 \mathrm{in}$. conduit to the respective preamplifier.

Volume II, Pages: 6.4-2) Figure 6.4-3 is a graph of normalized neutron flux and gamma flux in the J2 thimble at $62.5 \mathrm{MW}(\mathrm{t})$. The other "J" thimbles have similar characteristics. 


\subsubsection{Thermocouples}

Volume II, Pages: 6.9-1) Temperature conditions in the EBR-II primary system are monitored by an extensive network of thermocouples and resistance thermometers. The majority of these temperature sensors are thermocouples because of the lower cost and ease of installation of these units, compared to resistance thermometers. Resistance thermometers are installed in areas where greater accuracy in temperature measurement is desired, and it exceed the one than can be obtained using thermocouples.

In addition to the thermocouples and resistance thermometers, a small number of locally indicating temperature gages are installed on some primary sodium auxiliary systems.

Temperature sensors are installed on all of the important components in the primary system, with the exception of the reactor core and blanket subassemblies. It was not possible to install thermocouples on these subassemblies because of the design of the fuel handling system. Some indication of temperature conditions within these subassemblies is obtained by monitoring coolant outlet temperatures from certain subassemblies in the reactor. This is accomplished by mounting thermocouples on some of the subassembly holddown rods that extend downward from the underside of the reactor vessel cover. These temperature sensors are directly in the path of the outlet coolant stream from the selected subassemblies. All other components in the primary system contain temperature sensors. Considerable redundancy is employed in areas that become permanently radioactive after the start of reactor power operation. This redundancy assures the continued availability of temperature monitoring for all components even in the event of the loss of a few temperature sensors.

Volume II, Pages: 6.9-2) All of the thermocouples in the primary system and its auxiliary systems, are of the chromel-alumel type with $\mathrm{K}$ calibration, with the exception of those in the rotating plug freeze seals. These are iron-constantan thermocouples. Several types of thermocouple assemblies and assembly mountings are provided to satisfy the differing requirements of the various systems. A large number of thermocouples are required to operate in a high temperature and an intensely radioactive environment. Other thermocouples are installed in areas of low temperature and in non-radioactive environments. Both standard and special thermocouple assembly designs are provided to meet these requirements.

Volume II, Pages: 6.9-4) A standard type of thermocouple assembly is provided for monitoring temperatures on the outside surfaces of pipes and vessels in the primary and auxiliary systems. These thermocouples are supplied by Leeds \& Northrup and are shown in drawing No. B-875-452. A sketch of this type of assembly is shown in Figure 6.9-2. The unit is a spring loaded type with 20 gauge chromel alumel wires electrically insulated with fiberglass. The assembly is a Conax type, catalog no. SL-360-CA. The leads and fiberglass insulation, from the thermocouple assembly to the terminal head, is protected by a polyethylene sleeve.

Volume II, Pages: 6.9-4) The thermocouple assembly mounting consists of a 4-1/2 inch long, 1/4 inch diameter schedule 80 pipe nipple which is welded to the surface of a pipe or vessel. The thermocouple assembly is inserted into this pipe and screwed into the top of the mounting. The bottom tip, or hot junction, of the thermocouple is held in close contact with the surface of the sodium pipe by means of the spring in the assembly.

Volume II, Pages: 6.9-4) A number of thermocouples are mounted in thermowells, which extend into the fluid inside a pipe or vessel. The configuration of a thermocouple assembly of this type is shown in Figure 6.9-4, and is Leeds \& Northrup drawing No. B-8750456. A thermowell used in this type of application is shown in Figure 6.9-5.

Volume II, Pages: 6.9-4) The thermocouple assembly consists of 2 chromel and 2 alumel, 20 gage wires passing through Mgo electrical insulation, from the hot junctions to the terminal head. These 4 wires form two separate thermocouples which provide redundancy in a single thermocouple assembly. 
Volume II, Pages: 6.9-8) Two special thermocouples, which are designed for use in high temperatures and in high radiation environments, are shown in Figure 6.9-8. They are supplied by Leeds \& Northrup Co. and are shown in their drawing A-875-457. These thermocouples consists of 22 gage chromel, alumel wires which are either twisted and welded at the hot junction, or are butt welded.

Volume II, Pages: 6.9-8) The electrical insulation consists of a double wrap of fiberglass over each individual conductor and a single wrap of fiberglass over the pair of conductors. This insulation is covered by a 304 stainless steel braid wrapping.

Volume II, Pages: 6.9-8) These thermocouples are used on several components inside the primary tank. They are used to measure temperatures of the bulk sodium, of the nuclear instrument thimbles, of the coolant out of certain core and blanket subassemblies, and in several other applications.

Volume II, Pages: 6.9-25) Thermocouples in six of the eight instrument thimbles monitor air and structure temperatures in the J-1, J-3, J-4, 0-2, 0-3, and 0-4 thimbles. The thermocouples were removed from the J-2 and 0-1 thimbles when they were modified to become the Nuclear Instrument Test Facility. (Refer to Volume II, Chapter 7, for information concerning the NITF.) The thermocouples, located near the neutron detectors, are of the radiation- and temperature-resistant type.

Volume II, Pages: 6.9-25) Three thermocouples in each of the six thimbles provide outputs to the DAS, alarm, and a recorder. Four additional thermocouples monitor outlet air temperatures in the thimble exhaust ducts. All of thermocouples within the instrument thimbles are Type K (chromel-alumel) and pass out of the primary tank through disconnect plugs at the top of each thimble. All the thermocouple leads from the hot junction to the cold junction are chromel-alumel. However, all leads from the cold junction to the recorders, annunciator functions, etc., are copper.

Volume II, Pages: 6.9-29) The locations of the various thermocouples on the reactor vessel, and at the outlets of selected reactor subassemblies are shown in Figure 6.3-5.

Volume II, Pages: 6.9-29) All these thermocouples are of the high temperature and radiation resistant type, shown in Figure 6.9-8, since they are located in the immediate vicinity of the reactor.

Volume II, Pages: 6.9-29) Three different thermocouple systems are shown in Figure 6.3-5. They are the 503, the 1534 and the 540 series thermocouples. The 503 series thermocouples measure coolant temperatures at the outlet of a number of subassemblies in the reactor. The 1534 series measure coolant outlet temperatures from the reactor upper plenum. The 540 series monitor coolant temperatures in the high and low-pressure reactor plenum. The 503 series thermocouples are installed on the underside of the reactor vessel cover and are contained in 304 stainless steel sleeves. These thermocouples are fastened to the subassembly holddown "fingers" which extend downward from the bottom of the cover. The sleeves are located as close as possible to the outlets from certain core and blanket subassemblies. The chromelalumel leads from the hot junction pass upward through the reactor cover, through the two cover lifting columns to junction boxes at the top of each lifting column. The outputs from these thermocouples are distributed to a recorder, and to the reactor scram circuit.

Volume II, Pages: 6.9-31) Two thermocouples are located on the outlet coolant pipe from the reactor vessel. These are shown as items 1 and 2 in Figure 6.3-5 and are identified in the legend as numbers R1TC-1534-CG and R1-TC-1534-CF.

Volume II, Pages: 6.9-31) Thermocouple R1-TC-1534-CF is part of a circuit that measures the reactor coolant temperature difference. It provides the reactor coolant outlet temperature data to this circuit, which is described later in paragraph 6.9.3.6. Line diagrams for this circuit are shown in $\mathrm{H}$. K. Ferguson drawing P-461-M.

Volume II, Pages: 6.9-31A) Thermocouple R1-TC-507BK is used to sense the outlet coolant temperature from the reactor. It is mounted on the coolant inlet plenum to the intermediate heat exchanger. At position 3 of Figure 6.3-11 this thermocouples is connected to a cold junction. Output from the cold 
junction is transmitted via a rotary switch to a Leeds \& Northrup, M Line, millivolt-to-current converter. This type of circuit is described in paragraph 6.9.2.3.1. A block diagram of the circuit is shown in Figure 6.11-4 and a line diagram is given on H. K. Ferguson drawing P-461-S.

Volume II, Pages: 6.9-32) The 540 series thermocouples, shown in Figure 6.3-5, monitor coolant temperature in the high and low pressure plenum chambers beneath the reactor. They are used to monitor bulk sodium temperatures for input to the reactor safety shutdown circuit. The four thermocouples in this circuit are listed in Table 6.9-4.

Volume II, Pages: 6.9-32) The locations of some additional thermocouples on the reactor vessel and on the neutron shield are shown in Figure 6.3-6. All these sensors are of the temperature and radiation resistant type as shown in Figure 6.9-8. None of these thermocouples provide a scram or alarm function. Two thermocouples series are represented in Figure 6.3-6. The 507 series and the 540 series. The 507 series are read out on instrument No. P1-TIA-507, which is a Leeds \& Northrup, Speedomax G, Model D, 60,000 series recorder. It is located on the Primary Section Panel in the Control Room.

Volume II, Pages: 6.9-34) A number of thermocouples are installed on the primary coolant pump M-1 and M-2. The locations of these are shown in the instrument sensor drawing, Figures 6.3-7 and 6.3-8. A total of five thermocouples are installed on the drive motor of each pump to monitor temperatures. These thermocouples have no identifiable numbers and are read out on the control panel for each pump, in the power plant corridor panels.

Volume II, Pages: 6.9-34) The remaining thermocouples are located below the bulk sodium in the primary tank.

Volume II, Pages: 6.9-35) All of the other thermocouples in the figure have the 540 series designation and are read out on instrument No. R1-T1-540, which is located in Instrument Center No. 1 in the Reactor Containment Building. This instrument is a Leeds \& Northrup Speedomax G, Model D, 60,000 Series indicator.

Volume II, Pages: 6.9-36) Thermocouples R1-TC-540-H, K, M, N, and P, listed in Tables 6.9-7 and 6.9-8 are used to determine average bulk sodium temperature. Thermocouples R1-TC-1534-CF, which is described in paragraph 6.9.3.4.2, is used to determine the coolant outlet temperature form the reactor upper plenum. Figure 6.9-13 is a block diagram of these circuits showing the various components and readouts. In this diagram, signals from the bulk sodium average temperature circuit are combined with signals from thermocouple R1-TC-1534-CF, to produce a reactor coolant temperature rise signal.

Volume II, Pages: 6.9-38) The reactor outlet coolant temperature is detected by thermocouple R1-TC1534-CF, which is connected to a solid-state reference junction. The junction is connected to a Leeds \& Northrup, M-Line, Model 1990 millivolt-to-current converter located in Instrument Center No. 2 in the reactor building.

Volume II, Pages: 6.9-39) The temperature of the bulk sodium inside the primary tank is monitored by a number of thermocouples installed at various elevations and circumferential positions inside the tank wall. Figure 6.9-8 shows a typical thermocouple of this type.

Volume II, Pages: 6.9-39) Bulk sodium thermocouples are contained inside 5/16 inch diameter 304 stainless steel tubing which is attached to the inside wall of the primary tank. At the hot junction end of the tubing, the tube is bent $90^{\circ}$, and the tip extends approximately six inches into the bulk sodium from the tank wall.

Volume II, Pages: 6.9-40) Thermocouple R1-TC-530 is used as a temperature compensation sensor for the differential pressure type of primary tank bulk sodium level indicator. This thermocouple has no readout or scram function and it is described in the section on liquid level measurement as part of the primary tank bulk sodium level measurement description. 
Volume II, Pages: 6.9-41) The location of thermocouples on the walls and cover of the primary tank are shown in Figure 6.3-10. These thermocouples are designed to permit monitoring of the primary tank to detect temperature distributions inside the tank. Even more important is monitoring of temperatures in the primary tank cover. Uneven temperature distributions in the cover could cause distortion in the cover and possible misalignment of sliding or rotating mechanisms passing into the primary tank.

Volume II, Pages: 6.9-41) All of the temperature sensors shown in Figure 6.3-10 are of the temperature and radiation resistant type shown in Figure 6.9-8. None of the thermocouples in the figure have a scram or alarm function and all are read out on recorders. Four readout instruments record data from the thermocouples in Figure 6.3-10. They are P1-TIA-507, which is a Leeds \& Northrup Speedomax G, Model D, 60,000 series indicator. It is located in the Primary Section Panel in the Control Room.

Volume II, Pages: 6.9-44) All thermocouples in the primary heat exchanger are of the temperature and radiation resistant type shown in Figure 6.9-8. None of these sensors has an alarm or scram function.

Volume II, Pages: 6.9-44) Table 6.9-14 lists the thermocouples which are read out on indicator P1TIA-507. The line diagram for these thermocouple systems are given on H. K. Ferguson drawing P-461W.

\subsubsection{Pressure Measurement System}

Volume II, Pages: 6.10-1) Pressures in the primary and auxiliary systems are monitored by a number of different types of pressure measuring systems. The fluids being monitored by these systems are liquid sodium, liquid $\mathrm{NaK}$, silicone oil, argon gas, and air. Specially designed sensors are required to monitor sodium and $\mathrm{NaK}$ pressures, while more conventional equipment is used in contact with silicone and gases.

Volume II, Pages: 6.10-1) Pressure measurement systems are provided to monitor the following pressure conditions: (1) pressure differential between the inside and outside of the reactor containment building (2) pressures and differential pressures in the reactor vessel (3) primary pump pressures (4) primary sodium purification systems, and (5) pressures within the argon gas supply system. All of the above systems have remote readouts and some provide input to alarm circuits and the reactor scram circuit.

Volume II, Pages: 6.10-1) A differential pressure measuring system is provided to monitor the difference in pressure between the inside and outside of the reactor containment building. The location of these pressure sensors are shown as items 18 and 19 of Figure 6.3-4. Two separate differential pressure transmitters are provided as sensing elements in this system. They are Differential Pressure Transmitters Type $154 \mathrm{~W}$, manufacture by the Microsen Electronic Control Division of Manning, Maxwell, \& Moore Inc. The specification for the instrument are given in section $6.16(6.10 .2)$ of the paragraph 6.16 .

Volume II, Pages: 6.10-1) Pressure lines for the sensing instruments are attached to each side of the isolation valve shown in Figure 6.3-4. This pressure differential is sensed at the transmitters and electrical signals are distributed to a recorder and annunciators. The two transmitters are numbered R13-dPT641A and ... (To P.6.10-2)

Volume II, Pages: 6.10-2) (from P.6.10-1) ... R13-dPT641B. They provide signals to the recorder which is located on the Space Heating and Cooling System Panel in the Power Plant Corridor. The recorder is a Taylor Transcope Electronic Recorder, Model 701J. Specifications for this unit are given in section 6.16 (6.9.4.3) of paragraph 6.16.

Volume II, Pages: 6.10-2) A total of ten pressure sensors are installed on the reactor vessel. These sensors provide data on pressure drop across the core and the values of pressure at various positions within the reactor vessel. The locations of these sensors are shown in Figure 6.3-5. Data are provided for a recorder and the Reactor Scram Circuit. 
Volume II, Pages: 6.10-3) The sensing element for all theses pressure systems is a NaK filled volumetric pressure unit, manufactured by the Taylor Instrument Co. A sketch of the element is shown in Figure 6.10-2.

Volume II, Pages: 6.10-4) The pressure transmitters used in these systems are Taylor Transet Transducers, Model 750T, manufactured by the Taylor Instrument Co. The specifications of this transmitter and the NaK filled sensing element are given in section 6.16 (6.10.3.1) of paragraph 6.16.

Volume II, Pages: 6.10-8) All these pressure sensors except R1-PT-520A have failed and are no longer read out. This operating sensor is combined with R1-PT-522A to produce a value of pressure difference across the reactor, as described in paragraph 6.10.4. The output from R1-PT-520A is read out on a recorder on the primary-system panel in the control room. It is a 2-pen Leeds \& Northrup Speedomax M recorder. Specification of this type of recorder are given in section 6.16 (6.5.3.8) of paragraph 6.16. Line diagrams for these pressure systems are shown on H. K. Ferguson drawing No. P461-P

Volume II, Pages: 6.10-8) Three pressure sensors are installed on primary-sodium pump No. 1, and four on pump No. 2. Primary pump No. 1 contains sensors R1-PT-522-A, P1-PT-522-B, and R1-PT-522P. These are items 9, 10, and 11, respectively, of Figure 6.3-7. Only sensor R1-PT-522-A is still operational. Pressure sensors R1-PT-522-H, R1-PT-522-G, R1-PT-522-Q, and R1-PT-522-HX are installed on primary pump No. 2. These are items 31, 32, 33 and 43, respectively, of Figure 6.3-8. (Sensor R1-PT-522-HX was installed under ECN 420 and 422.) Only R1-PT-522-H and R1-PT-522-HX are still operational. Sensor R1-PT-522-A is combined in a special circuit with R1-PT-520-A to measure the pressure drop through the reactor. This circuit is shown in Figure 6.10-4.

Volume II, Pages: $6.10-8$ ) The pressure sensors, transmitters, and power supplies are of the same type as shown in Figures 6.10-2 and 6.10-3, and are described in paragraph 6.10.3. The power supplies for the transmitters are Transpac Model TR10/IT, and are located in Instrument Center No. 2 inside the reactor containment building. The transmitters for all the pressure sensors except R1-PT-522-A, R1-PT-522-H, and R1-PT-522-HX are in cabinet No. 7 of that instrument center. The transmitter for R1-PT-522-A is mounted under the reactor deck plates near primary pump No. 1; the transmitters for the other two sensors are mounted together under the reactor deck plates near primary pump No. 2. Each transmitter is connected to a Leeds \& Northrup M-Line millivolt-to-current converter. This converter is described in paragraph 6.9.2.3.1. Output from the converter for R1-PT-520-A is transmitted to a Rochester Model ET215 electronic trip unit. This unit is described in paragraph 6.4.6.5.1. The trip unit is connected to a relay in the reactor scram circuit.

Volume II, Pages: 6.10-10) Pressure sensors R1-PT-522-H and R1-PT-522-HX are located above the ball joint assembly in the piping of primary pump No. 2. The pressure-sensing heads, the transmitters, and the power supplies are all of the same type as those described in paragraph 6.10.3. The transmitters are connected to Leeds \& Northrup M-Line millivolt-to-current converters, described in paragraph 6.9.2.3.1. Output from the converter is transmitted to an indicator on the primary-system panel in the control room. No alarm or reactor scram function is associated with this circuit. The output from both R1-PT-522-A and R1-PT-522-H are read out on a Honeywell Electronic Electronik 17 2-pen recorder on the nuclear panel in the control room. Line diagrams for these pressure systems are presented in H. K. Ferguson drawing No. P-461-Z. The output from R1-PT-522-Hx is displayed by the digital data acquisition system (DAS) only.

Volume II, Pages: $6.10-10$ ) The primary sodium purification system contains a number of directpressure and differential-pressure measuring instrument systems. The purification system was recently modified, and additional pressure measuring instrumentation was added to the original instrument systems. The locations of all pressure sensors are shown in Figure 6.3-21. 
P. Volume II, Pages: 6.10-12) Two differential pressure measuring sensors, R1-dPT-548-B and G, are installed in the primary sodium purification system. A sketch of a typical sensor is shown in Figure 6.10-5, and a schematic of the differential pressure measuring system is shown in Figure 6.10-6.

Volume II, Pages: 6.10-12) This instrument is designed to measure differential pressure on processes having operating temperatures up to $1200^{\circ} \mathrm{F}$. The system consists of an electrical output transmitting section which is remote from the process, and diaphragm seal elements which are in contact with the liquid sodium. The diaphragm seals are connected to the transmitting section by means of two capillary tubes. The entire system is solid filled with $\mathrm{NaK}$ ( $78 \%$ potassium, $22 \%$ sodium eutectic). The diaphragm seals contact the sodium at the high and low pressure taps of the process piping. Pressures developed at the seals are transmitted by means of the NaK fluid to the transmitter section of the unit. Output of the instrument is linearly proportional to the pressure difference existing between the process pressure taps.

Volume II, Pages: 6.10-16) Pressure transmitter R1-PT-523-A is mounted on primary tank nozzle plug "N-1". It is designed to measure the pressure of the argon gas blanket inside the primary tank. This transmitter is a Leeds \& Northrup, Model 1912, Differential Pressure Transmitter, and specifications for the unit are presented in section $6.16(6.10 .6 .1)$ of paragraph 6.16. A block diagram of the circuit is shown in Figure 6.10-1. Output from the transmitter is distributed to two pressure indicators and to the alarm annunciator.

Volume II, Pages: 6.10-16) A pressure transmitter is installed on the outlet gas line from the two argon gas blowers that supply gas to the floating head tank. This transmitter is designated R6-PT-523-B, and is located at position 21 of Figure 6.3-23. It is a Manning, Maxwell, and Moore, Microsen Type 145 pressure transmitter. Specifications for this unit are presented in section 6.16 (6.10.6.2) of paragraph 6.16.

Volume II, Pages: 6.10-17) Two additional pressure measuring systems are installed on the argon gas supply system. The pressure transmitters are designated R6-PT-544-A, and R6-PT-544-B, and they are located at positions 20 and 22, respectively, in Figure 6.3-23. Both of these transmitters are Manning, Maxwell, and Moore, Microsen, Type 145 pressure transmitters. Specifications for these units are presented in section $6.16(6.10 .6 .2)$ of paragraph 6.16 .

Volume II, Pages: 6.10-22) Two pressure transmitters which measure pressure in the reactor upper plenum are located in the reactor-vessel instrument probe (refer to Section 6.15.11 for a description of that probe). One of these transmitters, PT-512A, feeds an instrument channel which provides a low-flow trip in the reactor shutdown circuit. Figure 6.10-7 is a block diagram of this instrument channel.

Volume II, Pages: 6.10-22) The pressure sensor, manufactured by Barton Instruments, consists of a sodium-filled line connected to one side of a differential pressure bellows. The bellows transmits the pressure signal (0-10psi) to a NaK-filled line that connects to the differential-pressure bellows in the transmitter case. The transmitter is a Barton Model 368. The Differential pressure bellows is connected to a strain gauge in the transmitter that converts the $0-10$ psi pressure signal to an electrical output. The transmitter produces a 4-20 mA output signal proportional to the input pressure. The other side of the differential pressure bellows is open to building pressure and is used for calibration by drawing a vacuum on the line. Having one leg open to the reactor building does not vary the pressure signal, since the pressure of the argon cover gas in the primary tank is held constant with respect to the reactor building by floating-head-tank pressure control.

\subsubsection{Flowmeter}

Volume II, Pages: 6.11-1) The flow of liquid metals is measured by magnetic flowmeters, flow tubes and ultrasonic flowmeters. The flow tubes were installed on piping in series with magnetic flowmeters in many cases. This was done for the purpose of calibrating the magnetic flowmeters after installation in the coolant system. It is possible for permanent-magnet flowmeters to have a linear output, and they are 
capable of a range of 100 to 1 . However, at the time these units were designed, the state-of-the-art required that they be calibrated in place in the piping system if acceptable accuracy was to be obtained. Differential-head-producing meters, such as flow tubes, have good accuracy but have a range of only about 10 to 1 . This is insufficient to cover the desire range of coolant flow in most liquid-metal systems. Therefore, the decision was made to install both types of flow sensors in most liquid metal piping systems where flow measurement is necessary. In this arrangement, the magnetic-flowmeter systems are assigned the main flow measurement task, and the differential-head-producing flow tubes are assigned the task of calibrating the magnetic flowmeters. This combination permitted measurement of sodium flow rate within $\pm 5 \%$ of actual values, over the entire range of flow.

Volume II, Pages: 6.11-1) Under Plant Modification No. 5099, one of the differential head flow elements, FE-541E, has been completely instrumented and is in continuous use. This instrument channel (FT-541E) measures total primary reactor flow and is described in paragraph 6.11.3.

Volume II, Pages: 6.11-4) A large magnetic flowmeter is installed in the piping leading from each of the primary sodium pump to the reactor high pressure plenum. These flowmeters are identical and are designated R1-FM-512A and R1-FM-512B. (See item 15, Figure 6.3-7 and item 37, Figure 6.3-8.) Flowmeter R1-FM-512A failed and is no longer read out. The other flowmeter is still operational. Specifications for the flowmeters are given in section 6.16. Some of the details of this type of unit are shown in Figure 6.11.1. These flowmeters were manufactured by the Mine Safety Appliance Research Corporation, their drawing No. 500725.

Volume II, Pages) 6.11-12: A small magnetic flowmeter is installed in the low pressure piping of the No. 2 primary sodium pump. The flowmeter is designated R1-FM-513-B. It is a 4 inch submersible flowmeter manufactured by Mine Safety Appliances (MSA) Research Corporation. Details of the flowmeter are shown on MSA drawing No. 500727, and specifications are given in section 6.16 (6.11.2.3) of paragraph 6.16. Figure 6.11-1 shows a sketch of some of the details of this type of flowmeter. Originally a second flowmeter (FM-513A) was installed in the low pressure plenum of primary pump No. 2 but it failed and was removed from the reactor shutdown system by passing its relay contacts in shutdown strings A and B. This was accomplished under Plant Modification No. 5239.

Volume II, Pages) 6.11-14: A magnetic flowmeter is installed on each of the primary tank shutdown coolers to measure the flow of $\mathrm{NaK}$ in these systems. The flowmeters are designated R4-FM-518-A and R4-FM-518-B and are shown as items 27 and 29, respectively, of Figure 6.3-18. They were manufactured by Mine Safety Appliance Research Corporation, and the details of the units are shown in drawing No. 500730. Specifications for the flowmeters are given in section 6.16 (6.11.2.4) of paragraph 6.16.

Volume II, Pages) 6.11-16: Two flowmeters are installed in the primary sodium purification system piping to measure the flow of sodium in the system. They are designated R1-FM-515-A and R1-FM-515$\mathrm{B}$, and their locations are shown in Figure 6.3-21. A sketch of this type of flowmeter is shown in Figure 6.11-8. These units are manufactured by Mine Safety Appliance Research Corporation and their dimensions are shown in MSA drawing No. 500729. Specifications for these flowmeters are given in section $6.16(6.11 .2 .5)$ of paragraph 6.16.

Volume II, Pages) 6.11-18: Five small magnetic flowmeters are installed on the sampling and plugging loops of the primary sodium purification system. These are designated R1-FM-709, and R1-FM710 on the plugging loop and three flowmeters, R1-FM-706, R1-FM-707 and R1-FM-708 on the sodium sampling loop.

Volume II, Pages) 6.11-19: Flowmeter R1-FM-710 measures the total flow through the plugging loop. It is read out on an indicator which is also on instrument center No. 3.

Volume II, Pages) 6.11-19: Flowmeters R1-FM-706 and R1-FM-708 measure the inlet and outlet flows from the sodium sampling loop. They are read out on indicators mounted on the sodium sampling 
and heater control panel in the basement of the reactor containment building. This panel is located at position 10 of Figure 6.2-58.

Volume II, Pages) 6.11-19: Flowmeter R1-FM-707 measures the bypass flow in the sodium sampling system. It is read out on an indicator at position 31 of Figure 6.9-30.

Volume II, Pages) 6.11-19: Magnetic flowmeter R1-FM-551 measures the flow of eutectic NaK in the secondary loop of the primary sodium purification system. The location of this flowmeter is shown on the flow diagram of Figure 6.3-28. This is a pipe mounted flowmeter of the type shown in Figure 6.11-8. It is manufactured by Mine Safety Appliances Research Corporation, and the details of the unit are shown on their drawing No. 500758. Specifications for the flowmeter are given in section $6.16(6.11 .2 .7)$ of paragraph 6.16.

Volume II, Pages) 6.11-20: The primary sodium coolant piping contains five flow tube type flowmeters. These are installed in the high and low pressure piping, and on the reactor coolant outlet piping. Flowmeters R1-FT-541-C, R1-FT-541-A and R1-FT-541-E are shown at positions 14, 17 and 20, respectively, in Figure 6.3-7. Flowmeter R1-FT-541-D and R1-FT-541-B are shown at positions 36 and 39, respectively of Figure 6.3-8. All of the flow tubes are of the "Gentile" design, and are manufactured by the Hammel-Dahl/Foster Engineering Division of the General Controls company.

Volume II, Pages) 6.11-20: A sketch of some of the details of a typical flow tube is shown in Figure 6.11-1. Fluid impact heads are positioned around the inside circumference of the tube on both sides of the venturi throat. Half of the impact heads face upstream and the other half face downstream. When sodium flows through the tube, a pressure difference develops across the throat of the tube. This pressure difference is transmitted by means of two sodium filled capillaries to a differential pressure sensing head. This head is identical to those used to detect differential pressures in primary system components, and is described in paragraph 6.10.5.2. These pressures are transmitted from the differential pressure sensing head to a differential pressure (dp) cell by means of two NaK filled capillaries. The dp cell actuates a Statham, Model G-1 strain gage transducer, which provides a signal to readout equipment. This transducer is described in paragraph 6.10.3.

Volume II, Pages) 6.11-20: These flow tubes were installed in the sodium coolant piping to provide a means of calibrating the magnetic flowmeters after their installation in the piping. A listing of these flow tubes, their location, and General Controls Co. drawing No. which show construction details, is presented in Table 6.11-1.

Volume II, Pages) 6.11-27: Under Plant Modification 5252, ultrasonic flow-measurement systems were added to the two low-pressure-plenum throttle valves (see Figure 6.11-16). The flowmeter systems were installed to partially restore some of the flow-measurement capabilities that were lost by failure of six of the original primary system flowmeters. Three of the Gentile-tube and three magnetic-type flowmeters had failed. These failures left the flow system of primary pump No. 1 without a functioning flowmeter.

\subsubsection{Level Measurement System}

Volume II, Pages: 6.12-1) Two sodium-level detectors were installed originally in the primary tank before it was filled with sodium. One was a spiral-resistance type probe, which was used for indication only. The other was a device based on measurement of static pressure head, but which incorporated a temperature sensor, or compensator, so that the device would indicate level changes resulting only from temperature changes. (With a constant sodium inventory in the primary tank, the static pressure head remains constant at different temperatures.) The latter device was used for indication and alarm.

Volume II, Pages: 6.12-1) The resistance probe worked only intermittently after it was first installed. Several problems are associated with its operation. Level is displayed in stepwise increments of several 
inches and is denoted by a series of lights, the number illuminated depending on the level sensed. Each level-indicating light is actuated by a relay that responds to the unbalancing of a bridge circuit, when sodium contacts the lower end of one of a series of probes placed at different levels. Because the resistance involved is small, the current must be relatively high to produce a usable unbalance voltage. Some of the more common problems have been: (1) The power supplies have been high-maintenance units, with filter and transformer failures causing the most frequent breakdowns; (2) the relays are sensitive to variations in supply voltage, are difficult to get, and are subject to drift of the settings with temperature; and (3) the probes are difficult to insulate, and breakdowns of the insulation have been frequent. Electrical problems have hampered the reliability of the unit, which is no longer operable and has not been used for several years.

Volume II, Pages: 6.12-4) The differential pressure type of level measuring sensor is still mounted in the F-2 nozzle of the primary tank cover, but it is no longer read out on any recorder or indicator.

Volume II, Pages: 6.12-4) The level sensor consists of a differential pressure sensor head identical to those described in paragraph 6.10.5.2 "Differential Pressure Measuring Systems". A sketch of the unit installed in the primary tank is shown in Figure 6.12-3. The sensing head is located at the bottom of the primary tank, beneath the bulk sodium. The high pressure side of the sensing head is open to the bulk sodium at the bottom of the tank. The low pressure side of the head is connected to a tube which is open to the inert gas blanket above the sodium in the primary tank. NaK filled ... (To P.6.12-6)

Volume II, Pages: 6.12-6) (from P.6.12-4) ... capillaries extend from the sensing head to a differential pressure cell and strain gage transducer which are located above the primary tank cover. These components, and the remainder of the circuit, are similar to those in the differential pressure measuring systems.

Volume II, Pages: 6.12-6) The level sensing unit is designated R1-LT-530 and is located at position 32 of Figure 6.3-9. Thermocouple R1-TC-530 was connected to this circuit to provide compensation for changes in the temperature of the bulk sodium.

Volume II, Pages: 6.12-6) A new bulk sodium level measuring system was recently installed in the primary tank. The level sensor was designated R1-LT-530R, and it was installed in the D-1 plug of the primary tank cover. The location of the plug is shown in Figure 6.3-24, and is item 34 of Figure 6.3-9.

Volume II, Pages: 6.12-6) A sketch of the level sensor installed in the primary tank cover plug is shown in Figure 6.12-4. This plug contains an extension tube which projects below the surface of the sodium to protect the buoyancy cylinder from local movement of sodium, and to limit the exposure of the internals of the transmitter to sodium aerosol that is in the argon-gas space. Just below the shield plug, the extension tube is vented to the argon space to eliminate pressure effects as the sodium level changes in the tube.

Volume II, Pages: 6.12-12) Additional details on this system are described in ANL-7623, "System for Measuring Sodium Level in EBR-II”, By J.B. Waldo and L.J. Christensen, October 1969.

Volume II, Pages: 6.12-12) A contact probe type of level indicator is installed in each of the two shutdown cooler surge tanks. Level indication is obtained by either a shorted or an open circuit condition between a probe and electrical ground. There are also considerably fewer probes on these sensors than on the unit in the primary tank. The locations of these level sensors are shown a position 26 and 28 in Figure 6.3-18, and are designated R5-LT-529-A and R4-LT-529-B.

Volume II, Pages: 6.12-12) There are three probes on each sensor, which consist of 304 stainless steel rods extending downward from their mounting flanges into the surge tank. These rods are 1/8 inch in diameter and have lengths of 2, 6 and 10 inches for each sensor unit. A spark plug, Champion Model no. OB-30X-3, is attached to the top of each rod outside the wall of the surge tank. A sketch of a typical 
probe installation is shown in Figure 6.12-6. A circuit diagram of the system is also shown in the figure because no H. K Ferguson line diagram of the system was available.

\section{Identification of Initial Conditions for the Available Recorded Data}

The scope of Task No.3 is the identification of a suitable pattern to identify the core isotopic composition at the moment of the start of the system recording to develop a faithful thermal-hydraulic model of the EBR-II plant. This is to refine the power level measurements to reduce uncertainties in flux levels and therefore burnup. Regarding to the core isotopic composition, it was calculated for each individual assembly, certainly for each of the experiment and for individual driver subassemblies. However, in the EBR-II system design descriptions reports, the information about the core isotopic composition was not found.

A significant amount of work was done to establish thermal power from the secondary sodium system by careful measurements of flow and temperature. This then had to be corrected for heat loss from other systems such as the shutdown coolers, auxiliary systems, etc. Consequently, it was able to reduce uncertainties to somewhere in the order of 3\% which was important for calculations of isotopic content. One other aspect of this is that prior to reprocessing, an assay is conducted of isotopic content of the fuel to compare with calculated values.

An important point is that the core isotopic composition had little or no effect on the plant performance and the thermal-hydraulic data that was retrieved. The outlet coolant mixes in the upper plenum and the intermediate heat exchanger so that any differences in power level among individual subassemblies is erased. In conclusion, the detailed core isotopic composition is not necessary for establishing the designated reactor power level from the core-averaged values.

In conclusion the third task was designed as an accessory task in case the isotopic composition would have affected the measure that were collected by the reactor data acquisition system. After careful analysis this seems not relevant information or at least the core loading pattern information and the assembly type information that is present in the run reports is deemed to be sufficient. The run reports have been digitized in most part in particular for the period in consideration is covered by the runs from 1985 to 1994.

\section{Summary}

In this report, the locations and drawings of the sensors in the EBR-II system and their library IDs such as EDMS ID and Alternate ID were summarized. The temperature, pressure, mass flow rate and fission rate in the EBR-II plant were measured and recorded by totally 931 sensors according to "ANL IFR OPERATIONS DIVISION Run Reports." which are currently available at INL Research Library (https://libhost.inel.gov/). Chromel-Alumel thermocouples and resistance thermometers were used to detect the temperature of the reactor system. Large and small magnetic flowmeters were used to measure the mass flow rate of sodium coolant. $\mathrm{NaK}$ filled volumetric pressure sensing element and differential pressure sensing element were used to detect the reactor pressure. Differential pressure type level transmitter and float type level transmitter were used to measure the sodium level in the primary tank. The storing location of the information of the sensors such as library IDs were also reported in the work. The information of core isotopic composition was not found in the EBR-II System Design Descriptions reports which are currently available at INL Research Library. However, it is identified that the previous work has done to establish thermal power from the secondary sodium system and is useful to develop a faithful thermal-hydraulic model of the EBR-II plant. The run reports are available at INL Research Library and the period in consideration is covered by the runs from 1969 to 1994. 


\section{References}

1. EBR-II, 1971, EBR-II System Design Descriptions - Volume I, “General Facilities,” Argonne National Laboratory, Argonne, Illinois, June 15.

2. EBR-II, 1971, EBR-II System Design Descriptions - Volume II, "Primary System,” Argonne National Laboratory, Argonne, Illinois, June 15.

3. EBR-II, 1971, EBR-II System Design Descriptions - Volume III, “Secondary System," Argonne National Laboratory, Argonne, Illinois, June 15.

4. EBR-II, 1971, EBR-II System Design Descriptions - Volume IV, "Steam System," Argonne National Laboratory, Argonne, Illinois, June 15.

5. EBR-II, 1971, EBR-II System Design Descriptions - Volume V, "Electrical System," Argonne National Laboratory, Argonne, Illinois, June 15.

6. EBR-II, 1971, EBR-II System Design Descriptions - Volume VI, "Sensitive Systems," Argonne National Laboratory, Argonne, Illinois, June 15. 